\title{
Amplituden- und phasenaufgelöste Polariton- Pulspropagation in GaAs bei niedrigen und mittleren Elektron-Loch-Plasmadichten
}

Dissertation zur Erlangung des Doktorgrades

der Mathematisch-Naturwissenschaftlichen Fakultäten

der Georg-August-Universität zu Göttingen

vorgelegt von

Joël Nägerl

aus Göttingen

Göttingen, September 1999 


\section{Inhaltsverzeichnis}

1. Einleitung 2

2. Theoretischer Hintergrund..................................................................... 4

2.1. Ausbreitung eines elektromagnetischen Feldes in Materie 4

2.2. Optische Eigenschaften des Halbleiters 5

2.2.1. Dielektrische Funktion einer Resonanz 6

2.2.2. Dielektrische Funktion des direkten Halbleiters 7

2.3. Das Exziton-Polariton 10

2.3.1. Polariton-Pulspropagation $\quad 13$

$\begin{array}{ll}\text { 2.3.2. Dephasierung } & 16\end{array}$

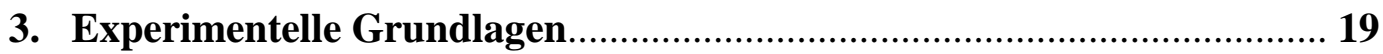

3.1. Gegenstand der Untersuchungen 19

3.2. Amplitude und Phase des elektrischen Feldes 21

3.3. Erzeugung kurzer Lichtpulse und spektrale Filterung 23

3.4. Methode der interferometrischen Kreuzkorrelation 26

3.4.1. Experimenteller Aufbau 27

3.4.2. Numerischer Auswertealgorithmus 29

3.4.3. Charakterisierung der Methode und Testmessungen 32

3.5. Pump- und Testexperimente 35

4. Experimentelle Untersuchungen zur Streuung

des Exzitons am ungebundenen e-h Plasma.......................................... 36

4.1. Einstrahlexperimente Polariton-Pulspropagation

$\begin{array}{ll}\text { bei niedriger und mittlerer Anregung } & 37\end{array}$

4.1.1. Bestimmung der Oszillatorstärke 38

4.1.2. Polariton-Pulspropagation bei niedrigen Anregungsdichten $\quad 39$

4.1.3. Polariton-Pulspropagation bei mittleren Anregungsdichten $\quad 42$

4.1.3.1. Bestimmung des relevanten Streukanals 43

4.1.3.2. Abhängigkeit der Dämpfung von der Anregungsdichte 45

4.1.3.3. Abhängigkeit der Phase von der Anregungsdichte 52

4.2. Anrege- und Testexperimente 57

4.2.1. Variation des zeitlichen Abstandes zwischen

Pump- und Testpuls $\quad 57$

4.2.2. Variation des Pumpspektrums $\quad 59$

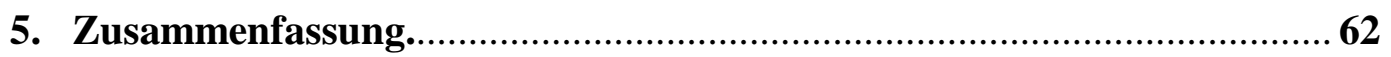

6. Wichtige Abkürzungen und Konstanten...............................................64

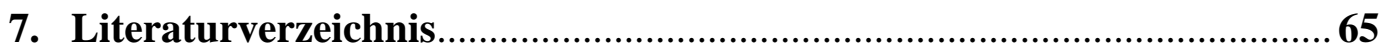

Danksagung

Lebenslauf 


\section{Einleitung}

Die optischen Eigenschaften eines direkten Halbleiters werden nahe seiner Bandlückenenergie von exzitonischen Effekten bestimmt. Die Wechselwirkung zwischen dem elektromagnetischen Feld und dem Halbleiter führt zur Bildung von selbstkonsistenten Anregungen, den Polaritonen. Nicht zuletzt wegen ihres großen Potentials für Anwendungen zur optischen Datenverarbeitung, der Speicherung und dem schnellen Transport von Information ist die kohärente Propagation der ExzitonPolaritonen Gegenstand intensiver Forschung: Flugzeitmessungen mit ps-Pulsen [1], solitonartige Propagation [2], charakteristische Propagationsschwebungen in der Amplitude [3] mit ihrer Abhängigkeit von Parametern wie Temperatur [4] oder Anregungsdichte [5] sind nur wenige Beispiele für die Vielfalt der Effekte, die mit der Polariton-Pulspropagation verbunden sind.

Die vorliegende Arbeit beschäftigt sich im wesentlichen mit zwei Dingen:

Zum einen wird die Methode der interferometrischen Kreuzkorrelation weiterentwickelt, mit der die Amplitude und Phase einer optischen Anwort einer Probe auf kürzesten Zeitskalen im sichtbaren Spektralbereich bestimmt werden können. Die Methode zeichnet sich durch ihre Sensitivität (bzgl. notwendiger minimaler Strahlungsleistung) und Dynamik (innerhalb einer Messung) vor anderen Methoden im Bereich der Ultrakurzzeitspektroskopie aus.

Zum anderen wird die Methode zur amplituden- und phasenaufgelösten Untersuchung der Exziton-Polariton-Pulsausbreitung am III-V Halbleiter Galliumarsenid eingesetzt. Ihre Phasenauflösung ist an Galliumarsenid-Volumenmaterial neu. Besonderes Interesse wird auf die Streuung des 1s-Exzitons am freien Elektron-Loch Plasma und die damit verbundene Auswirkung auf die Polariton-Pulspropagation gerichtet.

Die Arbeit gliedert sich wie folgt:

In Kapitel 2 werden die konstituierenden Gleichungen formuliert. Die elektromagnetischen Feldgleichungen (Kap. 2.1) ergeben zusammen mit den Materiegleichungen des Halbleiters (Kap. 2.2) die Polaritonen als Eigenzustände des optisch angeregten Halbleiters (Kap. 2.3). 
In Kapitel 3 wird die Probe beschrieben und die Meßgrößen sowie die Meßmethode zur ultraschnellen Amplituden- und Phasenmessung erläutert.

Kapitel 4 befaßt sich mit den experimentellen Ergebnissen, wobei zuerst auf Einstrahlexperimente eingegangen wird, in denen der Einfluß des Anregungsspektrums und der Anregungsfluenz auf die Polariton-Pulspropagation untersucht wird (Kap. 4.1). Anschließend werden vertiefende Experimente in Pump- und Test-Geometrien unter Variation des Pumpspektrums und des zeitlichen Abstandes zwischen Pumpund Testpuls vorgestellt (Kap. 4.2). 


\section{Theoretischer Hintergrund}

Dieses Kapitel befaßt sich mit der exzitonischen Elementaranregung in Halbleitern. Die Wechselwirkung von Exzitonen mit dem elektromagnetischen Feld führt auf Licht-Materie-Mischzustände, deren Eigenschaften im Grenzfall des linearen Regimes im Rahmen des Polaritonkonzeptes von Hopfield [17] beschrieben werden können. Nach Einführung der elektromagnetischen Feldgleichung (Teil 2.1) und den konstituierenden Bewegungsgleichungen des Halbleiters (Teil 2.2) wird das Polariton und seine Propagation diskutiert (Teil 2.3).

\subsection{Ausbreitung des elektromagnetischen Feldes in Materie}

Die Maxwellschen Gleichungen bilden die Grundlage für die Beschreibung aller klassischen elektromagnetischen Phänomene [6]. Zusammen mit den Materiegleichungen sind sie in der Lage, die klassische Dynamik wechselwirkender, geladener Teilchen und elektromagnetischer Felder vollständig zu beschreiben. Für den Fall eines unendlich ausgedehnten, nichtleitenden Mediums in Abwesenheit von Quellen und räumlich konstanter magnetischer Permeabilität und Suszeptibilität lassen sich aus den Maxwellgleichungen die Gleichungen für das elektrische Feld $\overrightarrow{\mathrm{E}}(\overrightarrow{\mathrm{r}}, \mathrm{t})$ ableiten [6]

$$
\nabla^{2} \overrightarrow{\mathrm{E}}(\overrightarrow{\mathrm{r}}, \mathrm{t})-\frac{1}{\mathrm{c}^{2}} \ddot{\overrightarrow{\mathrm{E}}}(\overrightarrow{\mathrm{r}}, \mathrm{t})=\frac{1}{\mathrm{c}^{2} \varepsilon_{0}} \ddot{\overrightarrow{\mathrm{P}}}(\overrightarrow{\mathrm{r}}, \mathrm{t})
$$

wobei c die Lichtgeschwindigkeit im Vakuum, $\varepsilon_{0}$ die Dielektrizitätskonstante des Vakuums und $\overrightarrow{\mathrm{P}}(\overrightarrow{\mathrm{r}}, \mathrm{t})$ die durch das elektrische Feld induzierte Polarisation der Materie ist.

Die Polarisation wird klassisch als die Verschiebungsdichte der Elektronen aus der Ruhelage definiert. Im atomaren Bild entspricht dieses dem Erwartungswert

$$
\vec{P}(t)=\int_{V} d^{3} x \cdot \psi^{*}(\vec{x}, t) \cdot \vec{d} \cdot \psi(\vec{x}, t)
$$

des Dipolopterators $\overrightarrow{\mathrm{d}}=(-\mathrm{e} \overrightarrow{\mathrm{x}})$ bezüglich der vom Atom eingenommenen, normierten Wellenfunktion $\psi(\vec{x})[7]$. 
Als Anwort der Materie auf das treibende elektrische Feld $\vec{E}\left(\vec{r}^{\prime}, t^{\prime}\right)$ verknüpft die Polarisation $\overrightarrow{\mathrm{P}}(\overrightarrow{\mathrm{r}}, \mathrm{t})$ den Zeitpunkt $\mathrm{t}$ mit allen früheren Zeitpunkten $\mathrm{t}^{\prime}$ sowie im Falle von räumlicher Dispersion den Ort $\vec{r}$ mit allen anderen Orten der Anregung $\vec{r}^{\prime}$.

\subsection{Optische Eigenschaften des Halbleiters}

Konzeptionelle Grundlage für die Beschreibung der optischen Eigenschaften der niedrigsten Elektron-Loch-Paaranregungen im linearen Regime ist das Oszillatormodell. Während dieses klassische Modell die optischen Eigenschaften einer isolierten Resonanz sehr erfolgreich beschreibt, erfordert die Beschreibung optischer Nichtlinearitäten insbesondere höherer Korrelationen eine quantenmechanische Behandlung. Die Halbleiter-Bloch-Gleichungen [7] stellen den quantenmechanischen Satz der konstituierenden Gleichungen des Halbleiters dar.

Im Fall von verschwindend kleinen elektrischen Feldstärken ${ }^{1}$ kann ein linearer Zusammenhang zwischen dem elektromagnetischen Feld und der Polarisation der Materie angenommen werden. Für ein homogenes Medium findet man [8]

$$
\overrightarrow{\mathrm{P}}(\overrightarrow{\mathrm{r}}, \mathrm{t})=\varepsilon_{0} \int_{\mathrm{V}} \mathrm{d} \overrightarrow{\mathrm{r}}^{\prime} \int_{-\infty}^{\mathrm{t}} \mathrm{dt}\left(\vec{\chi}\left(\overrightarrow{\mathrm{r}}-\overrightarrow{\mathrm{r}}^{\prime}, \mathrm{t}-\mathrm{t}^{\prime}\right) \cdot \overrightarrow{\mathrm{E}}\left(\overrightarrow{\mathrm{r}}^{\prime}, \mathrm{t}^{\prime}\right)\right.
$$

Die Suszeptibilität $\vec{\chi}\left(\overrightarrow{\mathrm{r}}-\overrightarrow{\mathrm{r}}^{\prime}, \mathrm{t}-\mathrm{t}^{\prime}\right)$ ist wegen der Homogenität translationsinvariant bezüglich des Raumes. Sie verschwindet wegen der Kausalität für $\mathrm{t}_{-} \mathrm{t}^{\prime}<0$. Prinzipiell von tensorieller Natur (Matrix 2. Stufe) kann sie für die unterschiedlichen Raumrichtungen unterschiedliche Einträge haben, ist aber für den Fall eines kubisch symmetrischen Mediums wegen der Separation der drei Raumrichtungen nur ein Skalar (Zur besseren Übersichtlichkeit wird der vektorielle Charakter von $\vec{E}$ und $\vec{P}$ im folgenden unterdrückt). Im Rahmen der linearen Antworttheorie sind der Realund der Imaginärteil der Suszeptibilität im Frequenzraum über die Kramers-Kronig-

1 „Klein“ bedeutet hier gegenüber inneren Feldern des Festkörpers. Im Falle des später betrachteten Exzitons kann über seine Energiedichte $\mathrm{w}_{\mathrm{ex}}=\mathrm{Ry} / \mathrm{a}_{\mathrm{b}}{ }^{3}$ (Rydbergenergie durch Exzitonenvolumen) und der Energiedichte eines elektrischen Feldes im Dielektrikum $\mathrm{w}_{\mathrm{EM}}=\varepsilon_{0} \varepsilon_{\mathrm{b}} \cdot \mathrm{E}^{2}$ ein kritisches elektrisches Feld E abgeschätzt werden. Für elektrische Felder vergleichbar oder größer als $\sim 10^{4} \mathrm{~V} / \mathrm{cm}$ sind nichtlineare Effekte der Exzitonresonanz zu erwarten. 
Transformation verknüpft, die von sehr allgemeiner Gültigkeit ist, da sie neben Linearität nur den kausalen Zusammenhang von dielektrischer Verschiebung und elektrischem Feld voraussetzt [6]. Der Realteil der Suszeptibilität beschreibt die „dispersiven“, der Imaginärteil die ,absorptiven“ Eigenschaften des Mediums.

\subsubsection{Dielektrische Funktion einer Resonanz}

Der Grund für die große Bedeutung des harmonischen Oszillators zur Beschreibung der dielektrischen Funktion einer isolierten Resonanz [9] liegt in der formalen Übereinstimmung seiner Ergebnisse mit denen, die aus quantenmechanischen Rechnungen an einem Zweiniveausystem hervorgehen [10].

Die dielektrische Funktion $\varepsilon(\omega, \overrightarrow{\mathrm{q}})=1+\chi(\omega, \overrightarrow{\mathrm{q}})$ einer isolierten Resonanz im Modell des harmonischen Oszillators ${ }^{2}$ ist gegeben durch

$$
\varepsilon(\omega, \overrightarrow{\mathrm{q}})=\varepsilon_{\mathrm{b}} \cdot\left(1+\frac{\mathrm{f}}{\omega_{0}(\overrightarrow{\mathrm{q}})^{2}-\omega^{2}-2 \cdot \mathrm{i} \gamma \cdot \omega}\right)
$$

Die Oszillatorstärke $\mathrm{f}$ beschreibt die Kopplungstärke des Oszillators an das treibende Feld. Sie ist das klassische Analogon zur quantenmechanischen Übergangswahrscheinlichkeit von Zustand A nach Zustand B. Sie ergibt sich aus dem Betragsquadrat des Übergangsmatrixelementes zwischen den zwei optisch gekoppelten Zuständen, deren (nackte) Energiedifferenz der Resonanzfrequenz $\omega_{0}(\vec{q})$ entspricht, wobei diese im Fall von räumlicher Dispersion vom Wellenvektor $\vec{q}$ abhängt. Mit der Dämpfung $\gamma$ werden summa summarum alle dissipativen Prozesse berücksichtigt. Die Dielektrizitätskonstante $\varepsilon_{\mathrm{b}}$ des Hintergrunds gibt den effektiven Beitrag höherer Resonanzen zur dielektrischen Funktion.

\footnotetext{
${ }^{2}$ Dieses Modell wird auch als „Drude-Modell“ oder „Thomson-Modell“ bezeichnet.
} 


\subsubsection{Dielektrische Funktion des direkten Halbleiters}

Im Fall eines Halbleiters mit direkter Bandlücke führt die Coulomb-Wechselwirkung über die Elektron-Loch-Korrelation zu einer Serie isolierter Resonanzen unterhalb sowie zu einem Absorptionskontinuum oberhalb der Bandkante. Die Beschreibung der optischen Eigenschaften der diskreten Resonanzen und des Kontinuums ist heuristisch mit einem Mehrfachoszillatormodell möglich. Um jedoch für die folgenden Experimente einen analytischen Ausdruck für die dielektrische Funktion des Halbleiters zu haben, wird ein fundierteres quantenmechanisches Modell herangezogen.

Ausgangspunkt hierfür ist ein Zweiband-Hamiltonoperator in Effektiv-Massennäherung [11]. Er besteht aus den Einteilchenenergien des Elektrons und des Loches (mit Massen $\mathrm{m}_{\mathrm{e}}$ und $\mathrm{m}_{\mathrm{h}}$ ) sowie der statisch abgeschirmten Coulomb-Wechselwirkung zwischen den beiden Elementarteilchen

$$
\mathrm{H}_{\mathrm{ex}}=\mathrm{E}_{\mathrm{g}}-\frac{\hbar \nabla_{\mathrm{e}}^{2}}{2 \mathrm{~m}_{\mathrm{e}}}-\frac{\hbar \nabla_{\mathrm{h}}^{2}}{2 \mathrm{~m}_{\mathrm{h}}}-\frac{\mathrm{e}^{2}}{4 \pi \varepsilon_{0} \varepsilon_{\mathrm{b}}\left|\overrightarrow{\mathrm{r}}_{\mathrm{e}}-\overrightarrow{\mathrm{r}}_{\mathrm{h}}\right|}
$$

Hierbei ist $\mathrm{E}_{\mathrm{g}}$ die Bandlückenenergie. Damit ist die Schrödingergleichung formal identisch mit dem des freien Wasserstoffatoms. Elliot konnte als erster eine Formel für den Imaginärteil der dielektrischen Funktion für $\omega>0$ angeben [12].

$$
\varepsilon_{2}(\omega)=\frac{A}{\omega^{2}}\left\{\sum_{n=1}^{\infty} 4 \pi R y^{3 / 2} \frac{1}{n^{3}} \delta\left(\hbar \omega-\hbar \omega_{g}+\frac{R y}{n^{2}}\right)+\frac{2 \pi \sqrt{R y} \cdot \Theta\left(\hbar \omega-\hbar \omega_{g}\right)}{1-\exp \left(-2 \pi \sqrt{R y /\left(\hbar \omega-\hbar \omega_{g}\right)}\right)}\right\}
$$

mit

$$
\mathrm{A} \equiv \frac{\hbar^{2} \mathrm{e}^{2}}{2 \pi \varepsilon_{0} \mathrm{~m}_{0}^{2}} \cdot\left(2 \mathrm{~m}_{\mathrm{red}} / \hbar^{2}\right)^{3 / 2}\left|\mathrm{~d}_{\mathrm{cv}}\right|^{2}
$$

der reduzierten Masse $\mathrm{m}_{\mathrm{red}}{ }^{-1}=\mathrm{m}_{\mathrm{e}}{ }^{-1}+\mathrm{m}_{\mathrm{h}}{ }^{-1}$ für die Relativbewegung des ElektronLochpaares, der Dielektrizitätskonstanten des Hintergrunds $\varepsilon_{\mathrm{b}}$, dem Matrixübergangselement $\mathrm{d}_{\mathrm{cv}}=\overrightarrow{\mathrm{e}} \cdot\langle\mathrm{c}|-\mathrm{i} \hbar \vec{\nabla}| \mathrm{v}\rangle$ des betrachteten optischen Überganges vom Valenzzum Leitungsband (c,v) parallel zur Polarisationsrichtung $\overrightarrow{\mathrm{e}}$, der Bandlückenenergie $\hbar \omega_{\mathrm{g}}$ und der Frequenz des elektrischen Feldes $\omega$. Die Rydbergenergie ist gegeben durch

$$
\mathrm{Ry}=\mathrm{m}_{\mathrm{red}} \mathrm{e}^{4} /\left(2 \hbar^{2}\left(4 \pi \varepsilon_{0} \varepsilon_{\mathrm{b}}\right)^{2}\right)
$$


Die Dispersionbeziehung des n-ten Exzitons lautet

$$
\hbar \omega_{\mathrm{n}}(\mathrm{q})=\hbar \omega_{\mathrm{g}}-\mathrm{Ry} / \mathrm{n}^{2}+(\hbar \mathrm{q})^{2} / 2 \mathrm{M}
$$

wobei $\mathrm{n}$ als Hauptquantenzahl die diskreten Resonanzen der Rydbergserie durchnumeriert. Die Parabel im Paarwellenvektor ${ }^{3} \mathrm{q}=\mathrm{q}_{\mathrm{e}}-\mathrm{q}_{\mathrm{h}}$ beschreibt die Schwerpunktsbewegung mit der Gesamtmasse $\mathrm{M}=\mathrm{m}_{\mathrm{e}}+\mathrm{m}_{\mathrm{h}}$. Der Bohrradius der s-Funktionen

$$
\mathrm{a}_{\mathrm{B}}=\mathrm{n}^{2} \cdot \hbar^{2} 4 \pi \varepsilon_{0} \varepsilon_{\mathrm{b}} / \mathrm{m}_{\mathrm{red}} \mathrm{e}^{2}
$$

steigt mit dem Quadrat der Hauptquantenzahl n. Die Oszillatorstärken $f_{\text {ex }}$ der diskreten Resonanzen sind proportional zum Betragsquadrats der Wellenfunktion im Ursprung der Relativkoordinate

$$
\mathrm{f}_{\mathrm{ex}} \propto\left|\psi\left(\mathrm{r}_{\mathrm{e}}-\mathrm{r}_{\mathrm{h}}=0\right)\right|^{2}
$$

und sind mit $1 / \mathrm{n}^{3}$ gewichtet. Das Absorptionskontinuum findet seinen Ausdruck oberhalb der Bandkantenenergie durch die Heavysidefunktion $\Theta\left(E-E_{g}\right)$.

Zwar beschreibt Elliots Gleichung die Lage und Stärke der Resonanzen korrekt, doch macht sie keine Aussagen über Form der Absorptionlinien, die hier $\delta$-förmig eingehen.

Es kann eine analytische Formel für die dielektrische Funktion angegeben werden, wenn eine lorentzförmige Verbreiterung angenommen und räumliche Dispersion vernachlässigt wird. Ausgehend von Elliots Formel Gl. 2.6 wird der Imaginärteil lorentzförmig um die Dämpfung $\Gamma$ verbreitert

$$
\varepsilon_{2}(\mathrm{E})=\frac{\Gamma}{\pi}\left\{\int_{0}^{\infty} \frac{\mathrm{dE}^{\prime} \varepsilon_{2}\left(\mathrm{E}^{\prime}\right)}{\left(\mathrm{E}-\mathrm{E}^{\prime}\right)^{2}+\Gamma^{2}}-\int_{0}^{\infty} \frac{\mathrm{dE}^{\prime} \varepsilon_{2}\left(\mathrm{E}^{\prime}\right)}{\left(\mathrm{E}+\mathrm{E}^{\prime}\right)^{2}+\Gamma^{2}}\right\}
$$

wobei die Verbreiterung derart vollzogen wird, daß die wegen des Kausalitätsprinzips erforderliche ungerade Parität von $\varepsilon_{2}(\omega)$ bezüglich der Energie erhalten bleibt [6].

\footnotetext{
${ }^{3}$ Der Paarwellenvektor q ist die Summe der Wellenvektoren des Valenz- und des Leitungsbandzustandes $\mathrm{q}_{\mathrm{f}} \mathrm{q}_{\mathrm{V}}+\mathrm{q}_{\mathrm{L}}$. In der Schreibweise für Elektronen und Löcher geht $\mathrm{q}_{\mathrm{V}}$ in $-\mathrm{q}_{\mathrm{h}}$ über.
} 
Mit der Formel

$$
\Gamma_{\mathrm{n}}=\Gamma+\frac{\Gamma_{1}-\Gamma}{\mathrm{n}^{2}}
$$

können unterschiedliche Dämpfungen für die verschiedenen Exzitonen berücksichtigt werden. Mittels Substitution und Partialbruchzerlegung kann der verbreiterte Imaginärteil berechnet werden. Durch Kramers-Kronig-Transformation wird der Realteil der dielektrischen Funktion bestimmt. Mit der Digammafunktion $\psi(\mathrm{x})$ [13] erhält man nach Tanguy [14]:

$$
\begin{aligned}
& \varepsilon(E)=\frac{2 \mathrm{~A} \sqrt{\mathrm{Ry}}}{\left(\mathrm{E}+\mathrm{i} \Gamma+\mathrm{i} \frac{\Gamma_{1}-\Gamma}{\mathrm{Ry}} \mathrm{E}_{\mathrm{g}}\right)} \cdot\left\{2 \psi\left(1-\mathrm{S}_{1}\right)+2 \psi\left(1+\mathrm{S}_{1}\right)-\left(1+\mathrm{i} \frac{\Gamma_{1}-\Gamma}{\mathrm{Ry}}\right) \cdot\left[\psi\left(1-\mathrm{S}_{2}\right)+\psi\left(1+\mathrm{S}_{2}\right)\right]+\right. \\
& \left.-\left(1-i \frac{\Gamma_{1}-\Gamma}{R y}\right) \cdot\left[\psi\left(1-S_{3}\right)+\psi\left(1+S_{3}\right)\right]\right\}+ \\
& +\frac{2 \mathrm{~A} \sqrt{\mathrm{Ry}}}{(\mathrm{E}+\mathrm{i} \Gamma)^{2}} \cdot\left\{\ln \frac{\mathrm{E}_{\mathrm{g}}^{2}}{\mathrm{E}_{\mathrm{g}}^{2}-(\mathrm{E}+\mathrm{i} \Gamma)^{2}}+2 \psi\left(\mathrm{S}_{4}\right)+2 \psi\left(\mathrm{S}_{5}\right)-4 \psi\left(\mathrm{S}_{1}\right)+\frac{1}{\mathrm{~S}_{4}}+\frac{1}{\mathrm{~S}_{5}}-\frac{2}{\mathrm{~S}_{1}}\right\} \\
& \text { mit } \quad S_{1}:=\sqrt{\frac{R y}{E_{g}}} \quad S_{2}:=\sqrt{\frac{R y+i\left(\Gamma_{1}-\Gamma\right)}{E_{g}-(E+i \Gamma)}} \quad S_{3}:=\sqrt{\frac{R y-i\left(\Gamma_{1}-\Gamma\right)}{E_{g}+(E+i \Gamma)}} \\
& \mathrm{S}_{4}:=\sqrt{\frac{\mathrm{Ry}}{\mathrm{E}_{\mathrm{g}}-(\mathrm{E}+\mathrm{i} \Gamma)}} \quad \mathrm{S}_{5}:=\sqrt{\frac{\mathrm{Ry}}{\mathrm{E}_{\mathrm{g}}+(\mathrm{E}+\mathrm{i} \Gamma)}}
\end{aligned}
$$

Die Lorentz-Linienform dieser Gleichung basiert zunächst auf keiner mikroskopischen Theorie. Experimentell beobachtete Feinheiten in der Linienform kann sie nicht erklären. Auch beantwortet sie nicht die Frage nach der Dämpfung des Kontinuums, die hier konstant für alle Frequenzen angenommen wird und für Hochdichteanregungen derzeit eine Herausforderung für die Vielteilchentheorie [15] darstellt. Wegen ihrer Analytizität bietet sie jedoch einen großen praktischen Nutzen für viele Anwendungen wie z.B. dem Design von Multischichtstrukturen, Festkörperlasern oder Mikrokavitäten [16] . 


\subsection{Das Exziton-Polariton}

Die Kopplung zwischen Exzitonen und dem elektromagnetischen Feld ist die Ursache für das Entstehen von Polaritonen, die Eigenschaften des Lichts mit Eigenschaften der Materie verbinden [17-19]. In der klassischen Betrachtung geschieht dies formal [20], indem die Gleichung für die Polarisation (Gl. 2.3) in die Gleichung für das elektrische Feld (Gl. 2.1) eingesetzt wird. Nach Born/Huang erhält man durch Fouriertransformation mit $\varepsilon(\omega, \vec{q})=1+\chi(\omega, \vec{q})$ eine Beziehung für transversale und eine Beziehung für longitudinale Wellen:

$$
\begin{array}{ll}
\text { transversal: } & \varepsilon\left(\omega, \overrightarrow{\mathrm{q}}_{\perp}\right)=\left(\frac{\mathrm{c} \cdot \overrightarrow{\mathrm{q}}_{\perp}}{\omega}\right)^{2}, \\
\text { longitudinal: } & \varepsilon\left(\omega, \overrightarrow{\mathrm{q}}_{\|}\right)=0
\end{array}
$$

wobei $\overrightarrow{\mathrm{q}}_{\perp}$ der Wellenvektor der transversalen Welle, des Polaritons, ist und $\overrightarrow{\mathrm{q}}_{\|}$den Wellenvektor der longitudinalen Welle ${ }^{4}$ beschreibt.

Durch die Kopplung zwischen dem Exziton und dem Lichtfeld verändern sich die Propagationseigenschaften der Polarisation des Exzitons grundlegend: Während die Propagation der Polarisation eines 1s-Exziton ohne elektromagnetischer Wechselwirkung nur unter Materietransport erfolgen kann, der einer parabelförmigen Dispersionbeziehung gehorcht, kommt einem Polaritonzustand durch seinen Wellencharakter ein zusätzlicher Energietransportkanal hinzu. Seine Transporteigenschaften sind implizit durch die Dispersionbeziehung (Gl. 2.14) gegeben.

Für eine isolierte Resonanz mit räumlicher Dispersion (Gl. 2.4), d.h. mit einer vom Wellenvektor abhängigen Resonanzfrequenz (s. Gl. 2.8), erhält man für transversale Wellen die in Abb. 2.1. gezeigte Dispersionsbeziehung $\omega(\mathrm{q})$ des Polaritons.

Der untere Teil des Realteils der Dispersionsrelation (unterer Polaritonast) beginnt bei q,$\omega=0$ mit (fast) konstanter Steigung $\hbar \cdot c / \sqrt{\varepsilon_{\text {stat }}}$ (lichtartiger Charakter) und schmiegt sich mit ansteigendem Wellenvektor an die Dispersionsbeziehung des Exzitons $\omega(\mathrm{q})$ an. Hier hat das Polariton exzitonischen Charakter.

\footnotetext{
${ }^{4}$ Longitudinale Lösungen der Maxwellgleichungen können von Licht bei senkrechtem Einfall nicht angeregt werden.
} 
Im Falle einer verschwindenden Dämpfung beginnt der obere Teil des Realteils der Dispersionsrelation (oberer Polaritonast) exzitonartig bei $\mathrm{q}=0$ und der longitudinalen ${ }^{5}$ Eigenfrequenz $\omega_{\mathrm{L}}$, krümmt sich nach links und geht dann wiederum in einen lichtartigen Teil über, der sich asymptotisch einer Gerade mit Steigung $\hbar \cdot c / \sqrt{\varepsilon_{\infty}}$ nähert.

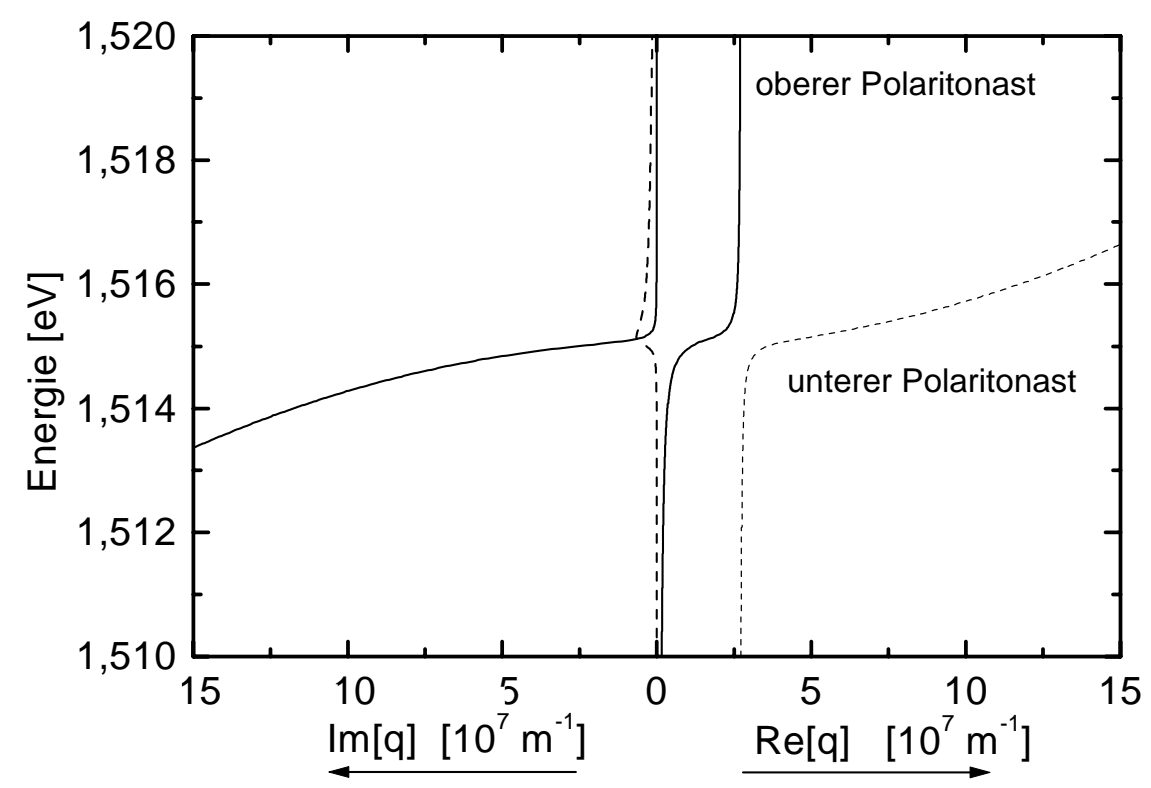

Abb. 2.1: Dispersionbeziehung des 1s-Exziton-Polaritonmodells mit räumlicher Dispersion und endlicher Dämpfung (Parameter: $\mathrm{f}=86 \mu \mathrm{eV}, \hbar \gamma=60 \mu \mathrm{eV}, \omega_{0}=1.515$ $\left.\mathrm{eV}, \mathrm{M}=0.5 \cdot \mathrm{m}_{0}\right)$. Der Realteil des Wellenvektors ist nach rechts, sein Imaginärteil nach links aufgetragen. Durchgezogen: oberer Polaritonast; gestrichelt: unterer Polaritonast.

Die Dielektrizitätskonstanten weit unter- und oberhalb der Resonanz, $\varepsilon_{\text {stat }}$ und $\varepsilon_{\infty}$, beschreiben die Abnahme des Brechungsindexes des Hintergrunds durch die Resonanz. Sie sind über die Lyddane-Sachs-Teller Relation [21] mit der transversalen Frequenz $\omega_{\mathrm{T}}$ (Frequenz für den Wellenvektor $\mathrm{q}=0$ ) und der longitudinalen Frequenz $\omega_{\mathrm{L}}=\omega_{\mathrm{T}}+\Delta_{\mathrm{LT}}$ verknüpft:

$$
\frac{\varepsilon_{\text {stat }}}{\varepsilon_{\infty}}=\frac{\omega_{\mathrm{L}}^{2}}{\omega_{\mathrm{T}}^{2}}
$$

wobei die sogenannte LT (longitudinal-transversal) Aufspaltung $\Delta_{\mathrm{LT}}$ im wesentlichen die Kopplungstärke des optischen Überganges bestimmt und die Oszillatorstärke

\footnotetext{
${ }^{5}$ Der Name longitudinale Eigenfrequenz ergibt sich aus Gl. 2.15 beim Auflösen nach $\omega$.
} 


$$
\mathrm{f}=\omega_{\mathrm{L}}^{2}-\omega_{\mathrm{T}}^{2}
$$

darstellt.

Der kritische Dämpfungsparameter, oberhalb dessen polaritonische Effekte verschwinden und die Absorptionformel in die des ungekoppelten Oszillators übergeht, ist gegeben durch $\gamma_{\text {krit }}=2 \omega_{0} \sqrt{\mathrm{f} \hbar \omega_{0} / \mathrm{Mc}^{2}}$. Für GaAs schätzt man ab $\hbar \gamma_{\text {krit }}=0.3 \mathrm{meV}$ [22].

Es ist bei dieser Betrachtung zu bemerken, daß wegen der Valenzbandentartung in Kristallen mit Zinkblendenstruktur zwei verschiedene Dispersionsrelationen, nämlich für das Leicht- und das Schwerlochband, zu erwarten sind. In Anlehnung an Arbeiten zur resonanten Brillouinstreuung in GaAs [23], bei denen eine „optisch gemittelte“ translatorische Masse erfolgreich zur Beschreibung der Daten eingesetzt wird, und zur Vermeidung zusätzlicher, den Sachverhalt verschleiernder Komplikationen soll jedoch auf diesen Aspekt nicht weiter eingegangen werden.

Die Dispersionsbeziehung Abb. 2.1 zeigt, daß im Falle räumlicher Dispersion zu jeder Energie prinzipiell zwei Polaritonmoden mit unterschiedlichen Wellenvektoren $\overrightarrow{\mathrm{k}}_{1}$ und $\overrightarrow{\mathrm{k}}_{2}$ existieren. Die Maxwellschen Randbedingungen, die ihren Ausdruck in den Fresnelschen Formeln finden [6],

$$
\begin{array}{ll}
\left.\mathrm{E}_{0}+\mathrm{E}_{\mathrm{R}}=\mathrm{E}_{1}+\mathrm{E}_{2} \quad \text { (Stetigkeit von } \mathrm{E}_{\text {tang }}\right) \\
\left.\mathrm{H}_{0}+\mathrm{H}_{\mathrm{R}}=\mathrm{H}_{1}+\mathrm{H}_{2} \quad \text { (Stetigkeit von } \mathrm{H}_{\text {tang }}\right)
\end{array}
$$

wobei E für das elektrische Feld und $\mathrm{H}$ für das Magnetfeld steht, die Indizes 0, R, 1 und 2 die eingestrahlte Welle, die reflektierte Welle, und die beiden Polaritonmoden im Medium bezeichnen, reichen nicht aus, um das Amplitudenverhältnis der beiden Moden festzulegen. Für einen vollständigen Satz von Randbedingungen fehlen weitere Bedingungen, die sogenannten zusätzlichen Randbedingungen ${ }^{6}$. Die Diskussion der ABC begann mit den Arbeiten von Pekard [24] und wurde lange kontrovers fortgesetzt [25-27]. Pekard forderte aufgrund phänomenologischer Überlegungen, daß der exzitonische Beitrag zur Polarisation an der Oberfläche für WannierExzitonen verschwinden soll.

$$
\mathrm{P}_{\mathrm{ex}}(\mathrm{z}=0, \omega)=0
$$

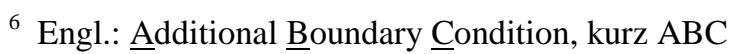


Obwohl dieser Ansatz aufgrund seiner mathematischen Einfachheit besticht und auch bis heute angewendet wird (s. z.B. [68]), darf nicht außer Acht gelassen werden, daß eine korrekte Beschreibung des Exziton-Polaritons eine mikroskopische, quantenmechanische Beschreibung des Polaritons in der Halbraumgeometrie erfordert [28].

\subsubsection{Polariton-Pulspropagation}

Da Polaritonen grundsätzlich bei optischen Anregungen isolierter Resonanzen relevant sind, sind ihre Propagationseigenschaften grundlegend und vielfältig in Halbleitern [29]. In der Literatur wird von verschiedensten Propagationseffekten berichtet: Von Flugzeitmessungen mit ps-Pulsen [1,30], Propagationsschwebungen [3] und Quantenschwebungen [31], ihrer Abhängigkeit von der Temperatur [4], der Anregungsdichte [5, 32], der Probengeometrie [33] oder dem Magnetfeld [34, 35], bis hin zu solitonartiger Propagation [2] oder selbstinduzierter Transmission [36]. Mögliche Anwendungen der Polariton-Pulspropagation zum Bau optischer Schalter sind unter [37] zu finden.

Durch einen einfallenden spektral schmalen ${ }^{7}$ Laserpuls der Zentralfrequenz $\omega$ wird ein Wellenpaket aus Polaritonzuständen mit Frequenzen dicht um die Zentralfrequenz $\omega$ erzeugt (siehe hierzu auch Abb. 2.1):

$$
\mathrm{E}(\mathrm{z}, \mathrm{t})=\frac{1}{\sqrt{2 \pi}} \int_{-\infty}^{+\infty} \mathrm{dk} \cdot \mathrm{A}(\mathrm{k}) \mathrm{e}^{\mathrm{i} \mathrm{kz}-\mathrm{i} \omega(\mathrm{k}) \cdot \mathrm{t}}
$$

(E: elektrisches Feld) wobei die Raumfrequenzamplituden durch

$$
\mathrm{A}(\mathrm{k}) \equiv \frac{1}{\sqrt{2 \pi}} \int_{-\infty}^{+\infty} \mathrm{dx} \cdot \mathrm{E}(\mathrm{z}, 0) \mathrm{e}^{\mathrm{ikz}}
$$

gegeben sind. Für den Grenzfall verschwindender Dämpfung bewegt sich dieses Paket entsprechend der Dispersionbeziehung mit der Gruppengeschwindigkeit $\mathrm{v}_{\mathrm{g}}(\omega)=\partial \omega /\left.\partial \mathrm{k}\right|_{\omega}$ durch die Probe $^{8}$.

\footnotetext{
7 „Schmal“ bedeutet hier gegenüber Strukturen in der Dispersionbeziehung.

${ }^{8}$ Für den Fall endlicher Dämpfung ist der Wellenvektor komplex und die Gruppengeschwindigkeit muß mit dem Pointingvektor berechnet werden.
} 
Für einen Laserpuls, dessen Spektrum breiter als die longitudinal-transversale Aufspaltung $\Delta_{\mathrm{LT}}$ ist, können beide Polaritonäste kohärent angeregt werden. Es werden Wellenpakete mit unterschiedlichen Energien und verschiedenen Gruppengeschwindigkeiten generiert. Als Folge erreichen zur selben Zeit jeweils zwei Wellenpakete das Ende der Probe 9 .

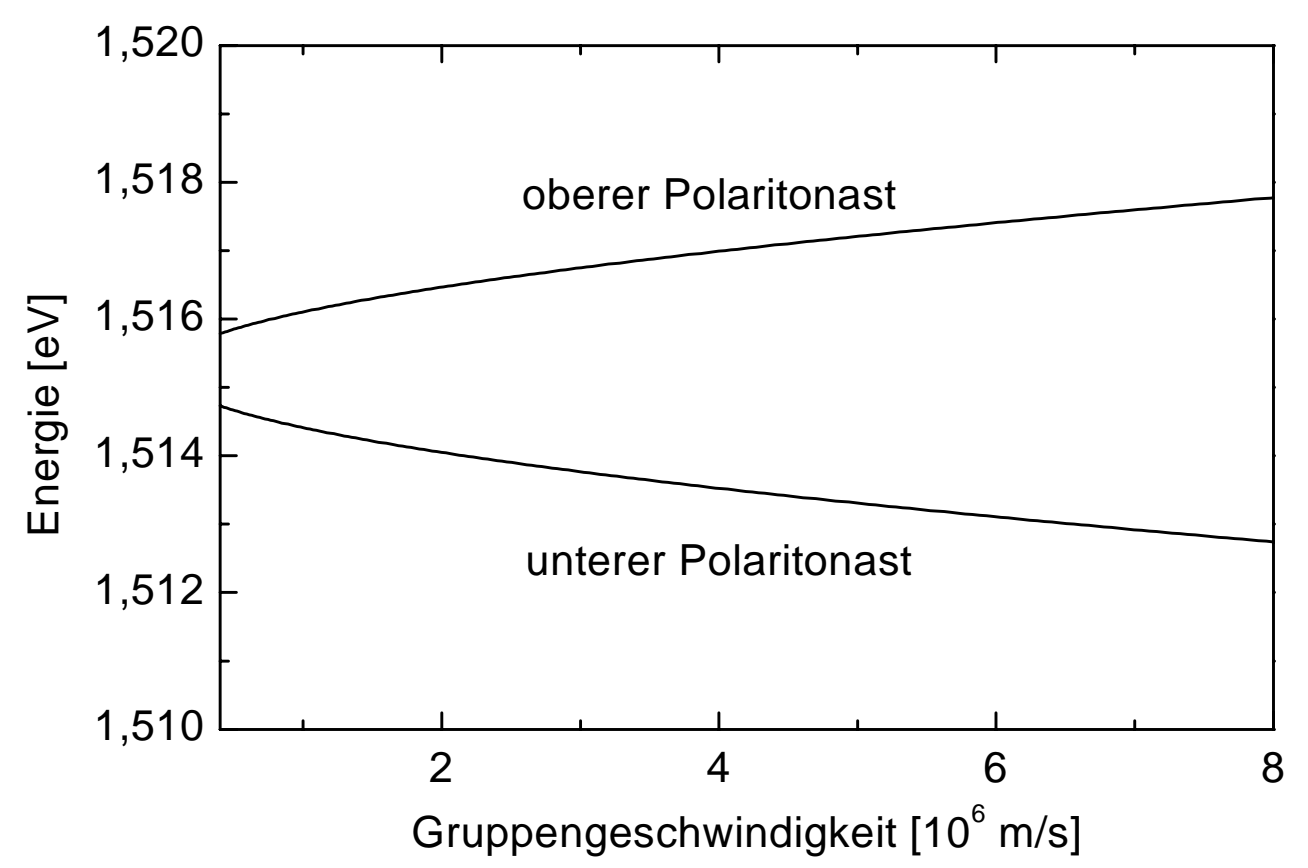

Abb. 2.2 Dispersion der Gruppengeschwindigkeit des oberen und unteren Polaritonastes.

Die Ankunft von jeweils zwei Wellenpaketen unterschiedlicher Frequenzen führt zu charakteristischen Schwebungen. Durch ihre relative Phasendifferenz $\Delta \phi(\tau, \mathrm{d})=\Delta \mathrm{k} \cdot \mathrm{d}-\Delta \omega \cdot \mathrm{d}$ und die damit verbundene, mit der Zeit kleiner werdende Energiedifferenz (s. Abb. 2.2) nimmt die Schwebungsperiodendauer mit der Zeit zu.

Ein analytischer Ausdruck für das zeitliche transmittierte elektrische Feld $\mathrm{E}(\mathrm{t})$ ist von Panzarini [38] mit Hilfe der Methode der stationären Phase [39] hergeleitet worden. Für den Fall eines $\delta$-Pulses und einer dielektrischen Funktion wie in (Gl. 2.4) ohne räumliche Dispersion (d.h. $\omega(q)=$ konst) erhält man

$$
E(t)=\frac{\left(2 d \cdot \omega_{T} \omega_{L T} / v\right)^{1 / 4}}{\sqrt{2 \pi} \cdot(t-d / v)^{3 / 4}} \cdot \exp (-\gamma(t-d / v)) \cdot \cos \left(\varphi_{0}\right) \cdot \cos \left(\Delta \varphi_{s}+\pi / 4\right)
$$

\footnotetext{
${ }^{9}$ Dieses gilt natürlich erst ab dem Zeitpunkt, wenn Wellenpakete vom unteren Polaritonast das Ende der Probe erreichen, welcher aufgrund des größeren Brechungsindexes unterhalb der Resonanz eine kleinere maximale Gruppengeschwindigkeiten aufweist als der obere Polaritonast.
} 
mit $\Delta \varphi_{\mathrm{s}}=\sqrt{\left(2 \mathrm{~d} \cdot \omega_{\mathrm{T}} \omega_{\mathrm{LT}} / v\right) \cdot(\mathrm{t}-\mathrm{d} / \mathrm{v})}$ und $\varphi_{0}=\omega_{\mathrm{T}} \cdot(\mathrm{d} / \mathrm{v}-\mathrm{t})-\mathrm{d} \cdot \omega_{\mathrm{LT}} /(2 \mathrm{v})$

wobei $v=c / n_{b}$ die Lichtgeschwindigkeit im Medium ist. Man erkennt, daß das elektrische Feld einem nicht exponentiellen Zerfall gehorcht. Der erste Kosinusterm beschreibt die schnellen Oszillationen mit der optischen Frequenz $\omega \approx \omega_{\mathrm{T}}$. Der letzte Term beinhaltet die charakteristischen Polariton Propagationsschwebungen. Die Amplitude wird als Funktion der Zeit und der Probendicke logarithmisch in Graustufen in Abb. 2.3 dargestellt. Deutlich zu erkennen sind die aperiodischen Propagationsschwebungen mit der zunehmenden Periodendauer für spätere Zeiten. Die Lage der Minima (schwarz) verschieben sich für zunehmende Probendicken zu früheren Zeiten.

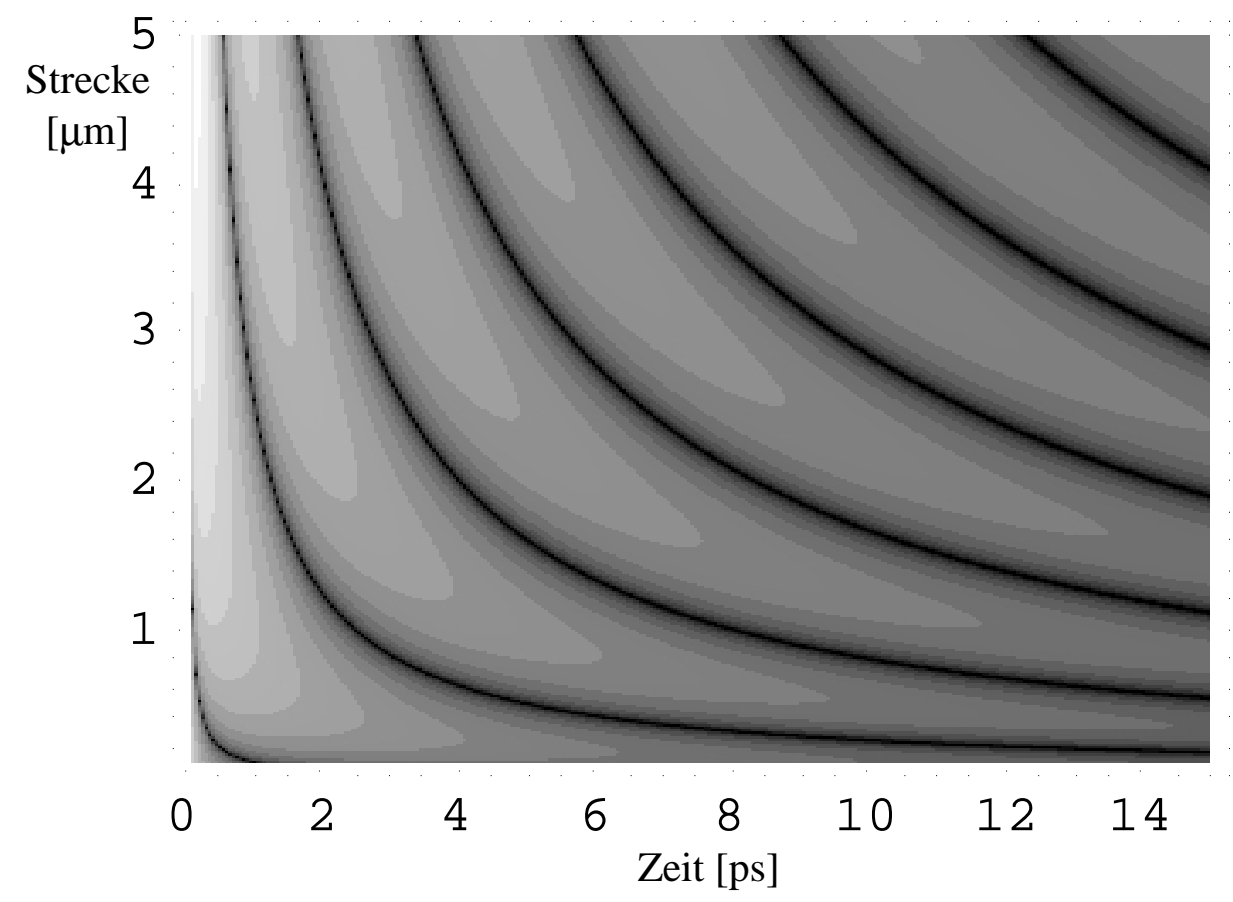

Abb. 2.3: Nach Gl. 2.23 berechnetes Raum-Zeitprofil der Propagationsschwebungen

Numerisch läßt sich das durch eine planparallele Platte der Dicke d transmittierte elektrische Feld $\mathrm{E}_{\mathrm{T}}(\omega)$ mit den Fresnelschen Randbedingungen berechnen. Mit dem komplexen Brechungsindex $\tilde{\mathrm{n}}=\sqrt{\varepsilon(\omega)}$, den Transmissionkoeffizienten an Vorderund Rückseite der Probe bzw. dem Reflexionskoeffizienten an der Rückseite $\mathrm{R}_{2}=(\tilde{\mathrm{n}}-1) /(\tilde{\mathrm{n}}+1)$ und dem einstrahlenden elektrischen Feld $\mathrm{E}_{0}(\omega)$ erhält man [40]

$$
E_{T}(\omega)=E_{0}(\omega) \cdot \frac{2}{\tilde{n}+1} \cdot \frac{2 \tilde{n}}{\tilde{n}+1} \cdot \frac{e^{i \tilde{n} \omega d / c}}{1-R_{2}^{2} \cdot e^{i 2 \tilde{\omega} \omega / c}}
$$




\subsubsection{Dephasierung}

Das Problem der Dephasierung ist in der zeitabhängigen Schrödingergleichung vorerst nicht enthalten, da sie mit der Kenntnis aller Anfangsbedingungen die zeitliche Entwicklung des Systems eindeutig voraussagt. Ist der Hamlitonoperator des Systems nur teilweise bekannt oder ist die Lösung eines bekannten Hamiltonoperators (z.B. aufgrund seiner Größe) nicht realisierbar, so kann nur ein Untersystem des Gesamtsystems erfaßt werden, und es werden Konzepte wie Streu- oder Relaxationszeiten nützlich. Zweckmäßigerweise unterscheidet man dann zwischen dem ,inneren“ System, welches von der Schrödingergleichung vollständig beschrieben wird, und dem ,äußeren“ System (dem „Bad“), über welches keine präzisere Information bekannt ist als über seine makroskopischen Parameter wie die Temperatur, das Potential oder ähnliche. Die Relaxations- oder Streuzeiten sind Ausdruck für die fehlende Information über das äußere System, welches mit dem inneren System Größen wie Energie und Impuls austauschen kann, und beschreiben die Endlichkeit der Lebensdauern der durch diese Kopplung bedingten (Quasi-) Eigenzustände des inneren Systems.

\begin{tabular}{|c|c|c|}
\hline $\begin{array}{c}\text { Kohärentes } \\
\text { Regime }\end{array}$ & Abkühlung & Rekombination \\
\hline $\begin{array}{l}\text { AC-Starkeffekt } \\
\text { Lochbrennen } \\
\text { Photonecho } \\
\text { kohärente LO- }\end{array}$ & $\begin{array}{l}\text { Heiße Ladungs- } \\
\text { träger, } \\
\text { Zwischental-/ } \\
\text { Intersubbandstreu }\end{array}$ & $\begin{array}{l}\text { Band zu Band } \\
\text { freie Exzitonen } \\
\text { gebundene } \\
\text { Exzitonen }\end{array}$ \\
\hline $\mathrm{T}_{2}$ & $\mathrm{~T}_{3}$ & $\mathrm{~T}_{1}$ \\
\hline
\end{tabular}

Heuristisch kann man die zeitliche Relaxation optischer Anregungen in Halbleitern in drei Regimes einteilen (Tab. 2.1) [41]. Das kohärente Regime umfaßt die kohärente Wechselwirkung von Feldern und Polarisationen. Beispiele sind der optische Starkeffekt [42], die kohärente Emission [43], das Photonecho [44] oder kohärente LO-Phononen-Streuung [45]. Die Zerfallszeit der Prozesse dieses Regimes ist die Dephasierungszeit $\mathrm{T}_{2}$. Die $\mathrm{T}_{1}$-Zeit beschreibt die Lebensdauer der Besetzungen. Für diese Zeiten gilt die Ungleichung $\mathrm{T}_{2} \leq 2 \cdot \mathrm{T}_{1}$. Der Faktor 2 stammt von dem Unterschied zwischen der Besetzung, welche proportional zum Betragsquadrat der 
Wellenfunktion $\sim|\psi|^{2}$ ist, und dem Zerfall der Kohärenz, der sensitiv auf die Wellenfunktion $\sim|\psi|$ selbst ist. Das Zwischenregime mit der charakteristischen $\mathrm{T}_{3}$-Zeit beschreibt die Abkühlung der Nichtgleichgewichtsverteilung der heißen Ladungsträger durch Energieaustausch mit dem Gitter [46].

Zur Illustration sind einige Streuprozesse in Abb. 2.4 schematisch wiedergegeben. Wichtige Streukanäle sind neben Ladungsträger-Ladungsträger-Streuung (e-e, e-h, hh) die Emission von LO-Phononen und die strahlende Rekombination. In diesem Bild nicht eingezeichnet sind Streuprozesse zwischen mehr als zwei Teilchen, wie z.B. Polarisationswellenstreuung oder Ladungsträger-Polarisationsstreuung [47, 48].

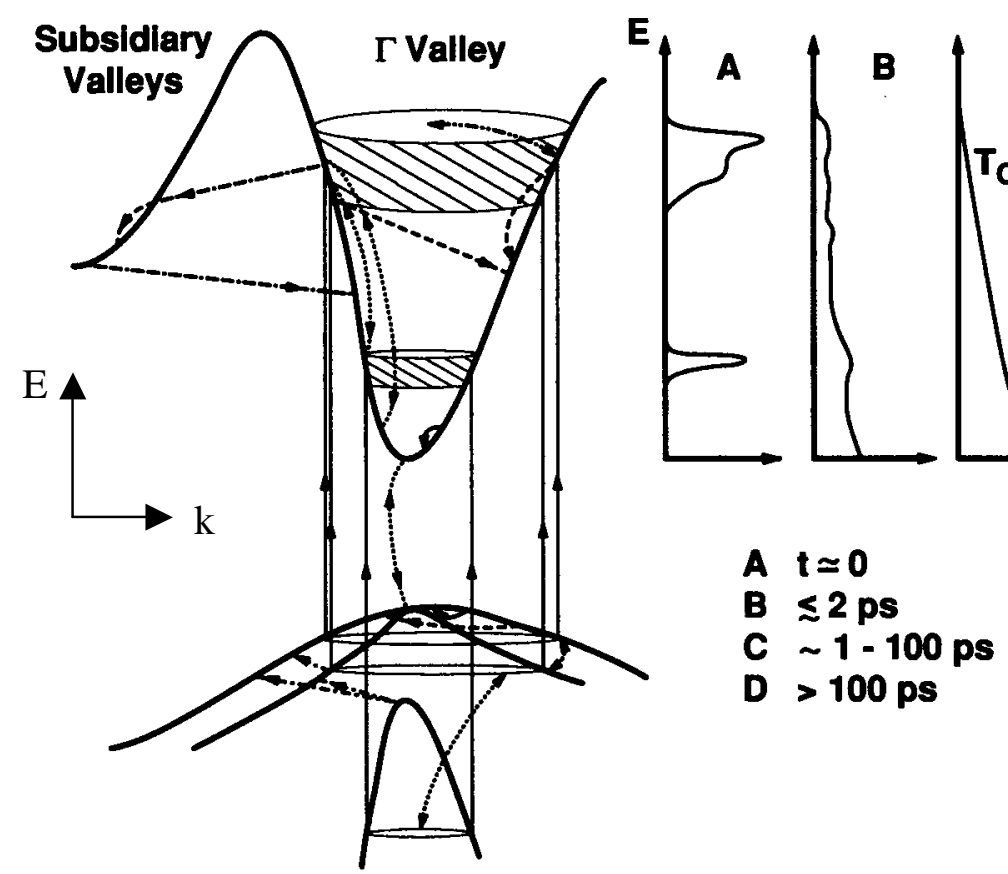

Abb. 2.4: Schematische Illustration der Relaxationsprozesse [84]. Doppelpfeile: Ladungsträger-Ladungsträger-Streuung (Impuls-Streuung für gestrichelt-doppelt-gepunktete Pfeile, Energie- und Impuls-Streuung für gestrichelt-gepunktete Pfeile), Einzelpfeile: Zwischental- und Intervalenzbandstreuung (gestrichelt-gepunkteter Pfeil), Ladungsträgeroptisches Phonon- oder Ladungsträger-gekoppelte-Phonon-Plasmon-Streuung (gestrichelter Pfeil) und Ladungsträger-akustisches-Phonon-Streuung (durchgezogener Pfeil). Die rechte Seite zeigt schematisch die anfängliche Elektronenverteilung und seine Zeitentwicklung.

Die Ladungsträger-Polarisationsstreuung spielt bei optischen Absorptionsspektren eine entscheidende Rolle. Mit ihr konnten zahlreiche Effekte wie die Form der Phononreplik nach gepulster Anregung [49], die niederenergetischen Ausläufer der Gainspektren [50], das Excitationbroadening [51] und das Umschalten von Absorption zu Gain in II-VI Quantentrögen [52] beschrieben werden. 
Bei der Beschreibung von Dephasierungsprozessen im Rahmen der HalbleiterBlochgleichungen geht die Selbstenergie der Ladungsträger ein, die die Renormalisierung der Einteilchenenergien aufgrund von Vielteilchenwechselwirkungen beschreibt [7]. Gewöhnlicherweise ist die Selbstenergie eine komplexe, frequenzabhängige Funktion, deren Realteil im statischen Grenzwert $\omega \mapsto 0$ die Verschiebung der Einteilchenenergien aufgrund der Vielteilchenwechselwirkung darstellt. Ihr Imaginärteil entspricht der inversen Dämpfungszeit und ist proportional zur homogenen Linienbreite. In Abb. 2.5 ist der von Manzke berechnete Imaginärteil der Selbstenergie für verschiedene Ladungsdichten mit der Temperatur $77 \mathrm{~K} \mathrm{zu}$ verschwindendem Impuls der Teilchen aufgetragen. Durch den starken Anstieg der Dephasierung zur Bandkante hin erhält man an der Exzitonresonanz (ein Rydberg unterhalb der Bandkante) einen Gradienten, der $\mathrm{zu}$ einer asymmetrischen Exzitonlinienform führt. Für steigende Anregungsdichte nimmt die Asymmetrie der Exzitonresonanz zu.

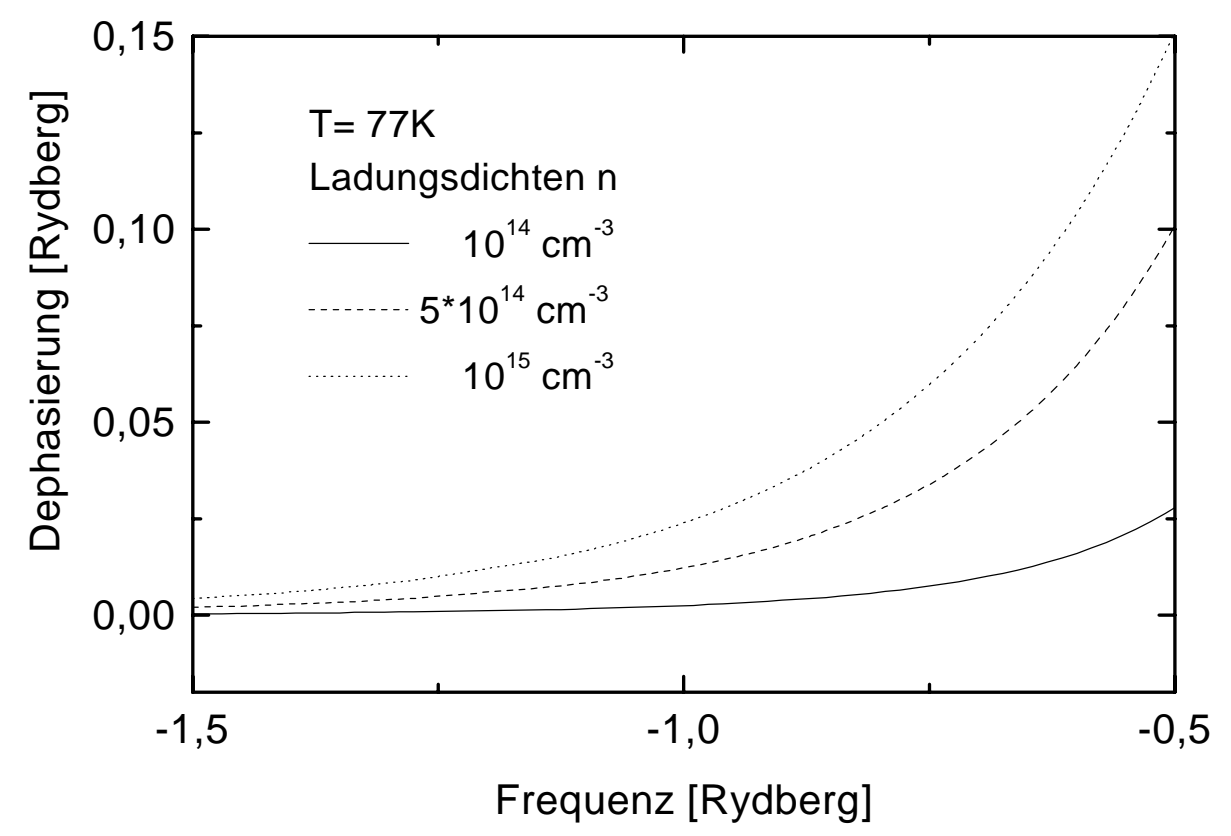

Abb. 2.5: Dephasierung in Abhängigkeit von der Energie für drei unterschiedliche Anregungsdichten bei $\mathrm{T}=77 \mathrm{~K}$ zum Wellenvektor $\mathrm{k}=0$. Die Bandkante liegt bei 0, das Exziton eine Rydbergenergie tiefer [83]. 


\title{
3. Experimentelle Grundlagen
}

\subsection{Gegenstand der Untersuchungen}

Die Berechnung der Kinetik des optisch angeregten Halbleiters ist mit erheblichem numerischen Rechenaufwand verbunden, so daß Näherungen gesucht werden müssen, die zum einen die physikalischen Effekte im Halbleiter beschreiben können, zum anderen aber noch rechnerisch handhabbar sind [15]. Zur Untersuchung der Streuung der Kontinuumsexzitonen am 1s-Exziton sind deshalb Proben nützlich, die sowohl einen hohen Grad an struktureller Perfektion aufweisen, um störende Effekte wie Streuung an geladenen oder neutralen Störstellen vernachlässigen zu können, als auch Volumeneigenschaften haben, um den Einfluß der Oberfläche (inhomogene Verbreiterung durch Unordnung) zu minimieren. Je kleiner die 1s-Exziton Linienbreiten sind, desto kleiner sind die minimalen Anregungsdichten, deren Einfluß auf das Exziton untersucht werden kann.

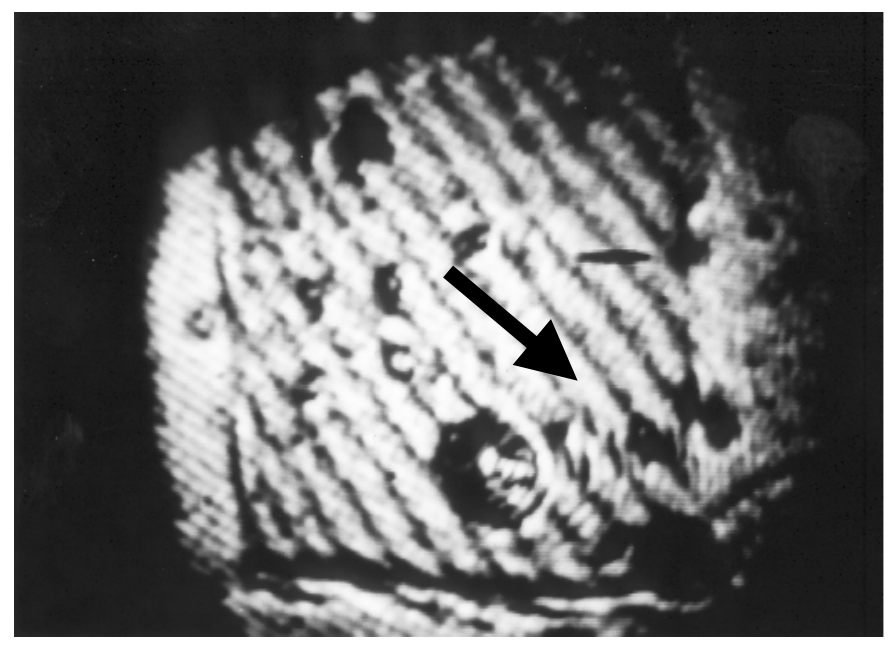

\begin{abstract}
Abb. 3.1: Photo von der Probe bei Beleuchtung von hinten. Die Messungen wurden an der angegebenen Stelle durchgeführt. Man erkennt das Streifenmuster, das durch Mehrfachreflexionen im Inneren der Probe zustande kommt.
\end{abstract}

Die untersuchte Probe ist ein $0,5 \times 0,5 \mathrm{~mm}^{2}$ großes Stück Galliumarsenid (III-V Halbleiter in Zinkblendestruktur, Photo s. Abb. 3.1), das durch Gasphasenepitaxie gewonnen wurde und eine fast kompensierte Konzentration von flachen Akzeptoren und Donatoren $\left|\mathrm{N}_{\mathrm{d}}-\mathrm{N}_{\mathrm{a}}\right|=2 \cdot 10^{14} \mathrm{~cm}^{-3}$ aufweist [34]. Die Probe ist keilförmig mit einer 
Dicke von 3.6 bis $4 \mu \mathrm{m}$ und wurde interferometrisch mit einem Spektrometer ausgemessen ${ }^{10}$.

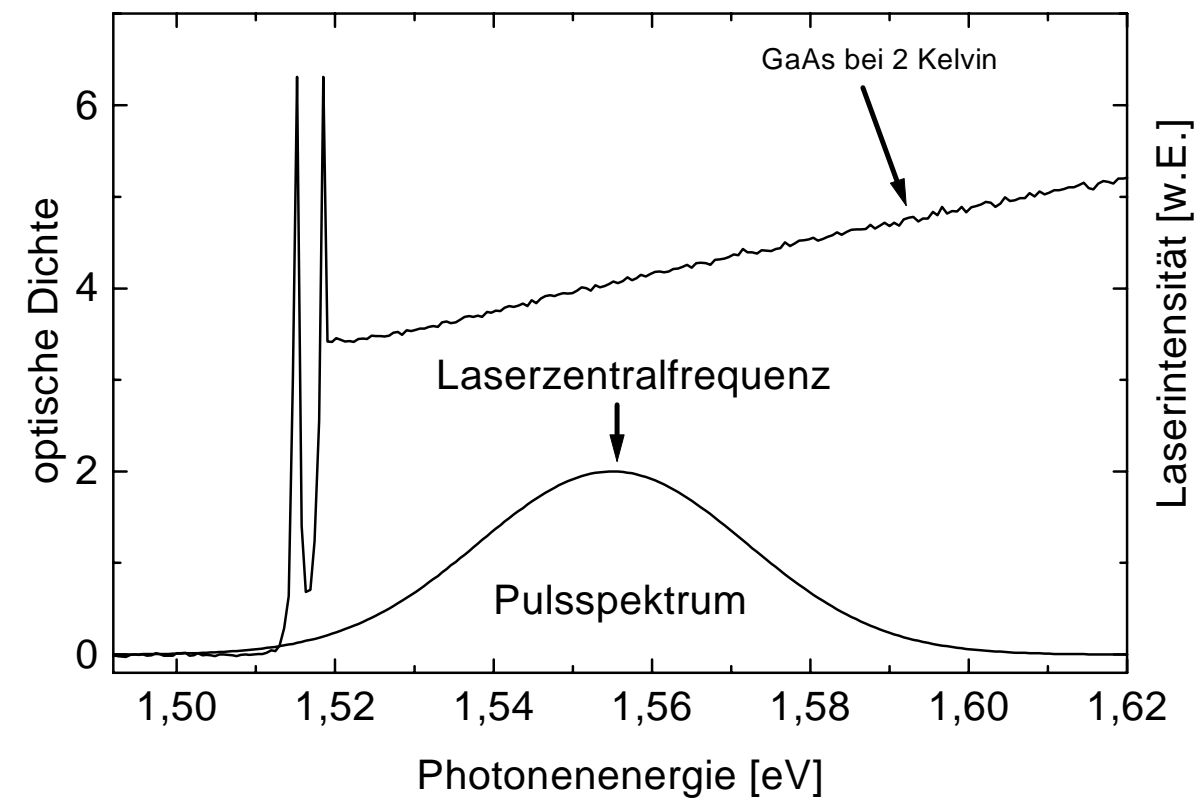

Abb. 3.2.a: Absorptionsspektrum $\alpha(\omega) \cdot d$ der GaAs Probe (d: Probendicke), welches mit Weißlicht aufgenommen wurde, und ein typisches, gauß̈örmiges Spektrum eines Laserpulses.

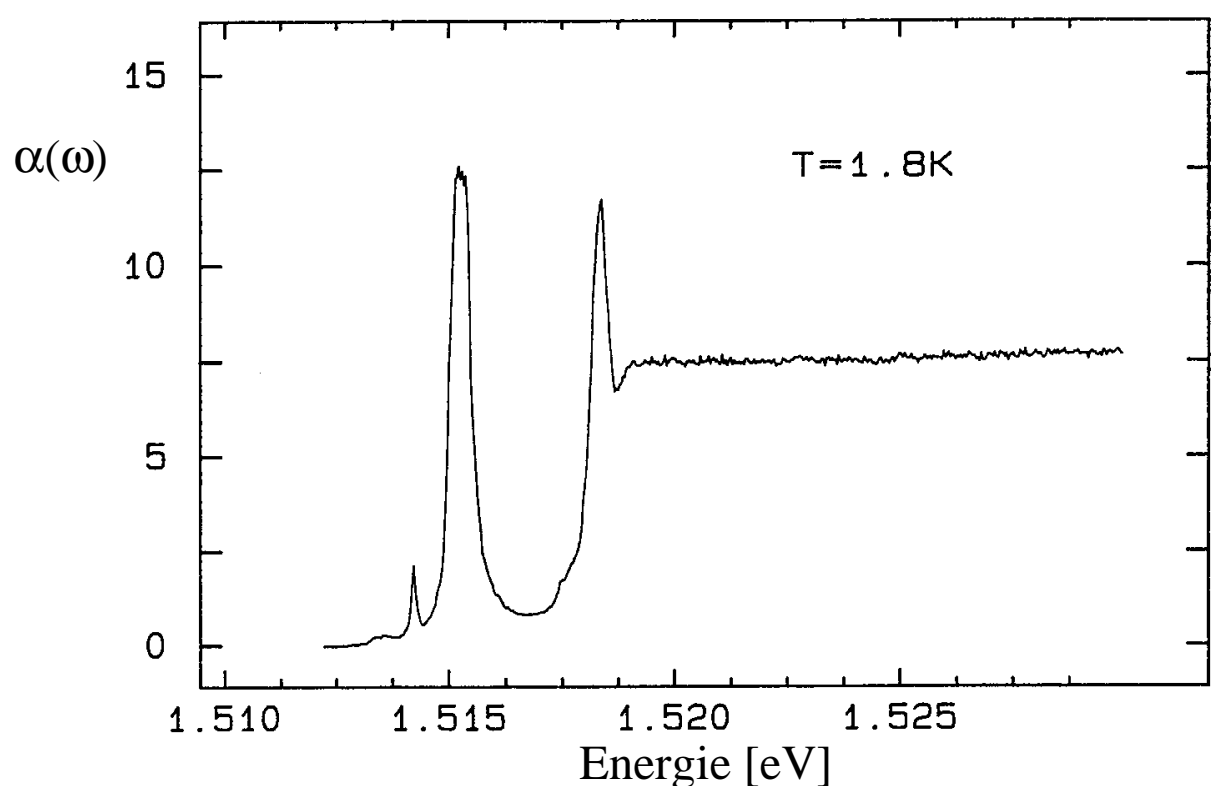

Abb. 3.2.b: Absorptionsspektrum $\alpha(\omega)$ für einen kleineren Energiebereich. Die Exzitonlinien sind nach oben hin bei $\alpha(\omega) \approx 13$ wegen Lumineszenz und Detektorrauschen begrenzt.

\footnotetext{
${ }^{10}$ Spektral gefiltertes Licht wird auf die Probe gesendet, die in Transmission auf eine IR Kamera abgebildet wird. Aus der Lage der Interferenzstreifen für unterschiedliche Frequenzen kann mit der
} 
Das mit Weißlicht aufgenommene optische Dichtespektrum bei 2 Kelvin (s. Abb. 3.2.) weist eine ausgeprägte 1 s- sowie 2 s-Exzitonresonanz mit dem sich anschließenden Kontinuum auf. Die Absorptionsspitzen der 1s- und 2s- Exzitonen sind wegen Lumineszenz und Detektorrauschen nicht aufgelöst. Die Bandkante liegt bei 1.5192 $\mathrm{eV}$, das 1s-Exziton um ein Rydberg (4.2 meV) tiefer und der Bohrradius ${ }^{11}$ beträgt $\sim 12 \mathrm{~nm}$. Unterhalb des 1s-Exzitons liegt das am Donator gebundene Exziton. Hochaufgelöste Absorptionsmessungen im Kontinuum zeigen eine schwache LOPhononreplik bei einer LO-Phononenergie (36 meV) oberhalb des 1s-Exzitons [53]. Zusätzlich zu dem optischen Dichtespektrum ist ein typisches Laserspektrum mit $\hbar \omega_{\text {central }}=1.555 \mathrm{eV}$ der verwendeten 40 fs Pulse eingezeichnet.

\subsection{Amplitude und Phase des elektrischen Lichtfeldes}

Zum Verständnis der folgenden Meßgrößen sollen die Begriffe Amplitude und Phase definiert werden.

Jedes zeitlich veränderliche elektrische Feld eines Laserpulses kann dargestellt werden als Produkt einer langsam veränderlichen Amplitude $\mathrm{A}(\mathrm{t}) \geq 0$ und einer phasenmodulierten Schwingung

$$
E(t)=A(t) \cdot \cos \left(\omega_{T} \cdot t+\varphi(t)\right)
$$

mit der zeitabhängigen Phase

$$
\varphi(t)=\varphi_{0}+\Delta \omega \cdot t+\alpha \cdot t^{2}+\beta \cdot t^{3}+\ldots
$$

Die Konstante $\varphi_{0}$ legt den Zeitnullpunkt fest, $\omega_{\mathrm{T}}$ ist die Trägerfrequenz und $\Delta \omega$ ist äquivalent zu einer Verschiebung zu einer neuen Trägerfrequenz $\omega_{\mathrm{T}^{\prime}}{ }^{\prime}=\omega_{\mathrm{T}}+\Delta \omega$. Die Terme $\alpha \cdot t^{2}$ und höhere Ordnungen beschreiben eine sich verändernde Momentanfrequenz und werden als „Chirp“ bezeichnet. Mit einem Phasensprung meint man die schnelle zeitliche Änderung der Phase wie sie in Abb. 3.3 dargestellt ist. Diese Darstellung ist eindeutig bis auf den Fall eines Phasensprungs um $\pi$, bei dem sowohl $+\pi$ als auch $-\pi$ denselben zeitlichen Verlauf des elektrischen Feldes wiedergeben.

Kenntnis des Brechungsindex die Probendicke bestimmt werden.

${ }^{11}$ Bei dem angegebene Wert wird eine „optisch gemittelte“ Valenzbandmasse angenommen. Wegen der unterschiedlichen Massen des hh- und lh- Bandes existieren zwei Bohrradien (hh: $11 \mathrm{~nm}$, lh: 18 $\mathrm{nm})$. 


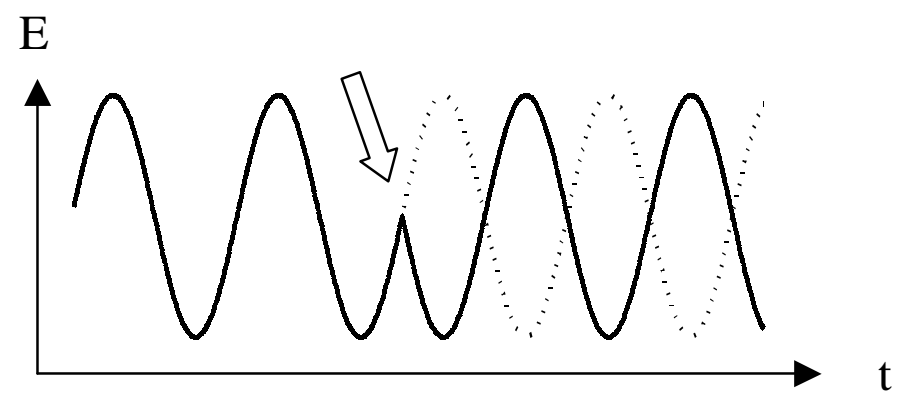

Abb. 3.3: Phasensprung um $\pi$ (Pfeil)

Ein Phasensprung entsteht beispielsweise bei einer Schwebung. Werden zwei Primärschwingungen mit Frequenzen $\omega_{1}$ und $\omega_{2}$ und Amplituden $A_{1}$ bzw. $A_{2}$ überlagert, so daß

$$
\begin{aligned}
E(t) & =A_{1} \cdot \cos \left(\omega_{1} t\right)+A_{2} \cdot \cos \left(\omega_{2} t\right) \\
& \equiv A(t) \cdot \cos \left(\omega_{T} \cdot t+\varphi(t)\right),
\end{aligned}
$$

erhält man für den Fall gleich großer Amplituden $A_{1}=A_{2}$ eine Phase $\varphi(\tau)$ mit stufenförmigen Phasensprüngen von exakt $\pm \pi$. Die mittlere Steigung der Phase ${ }^{12}$ ohne

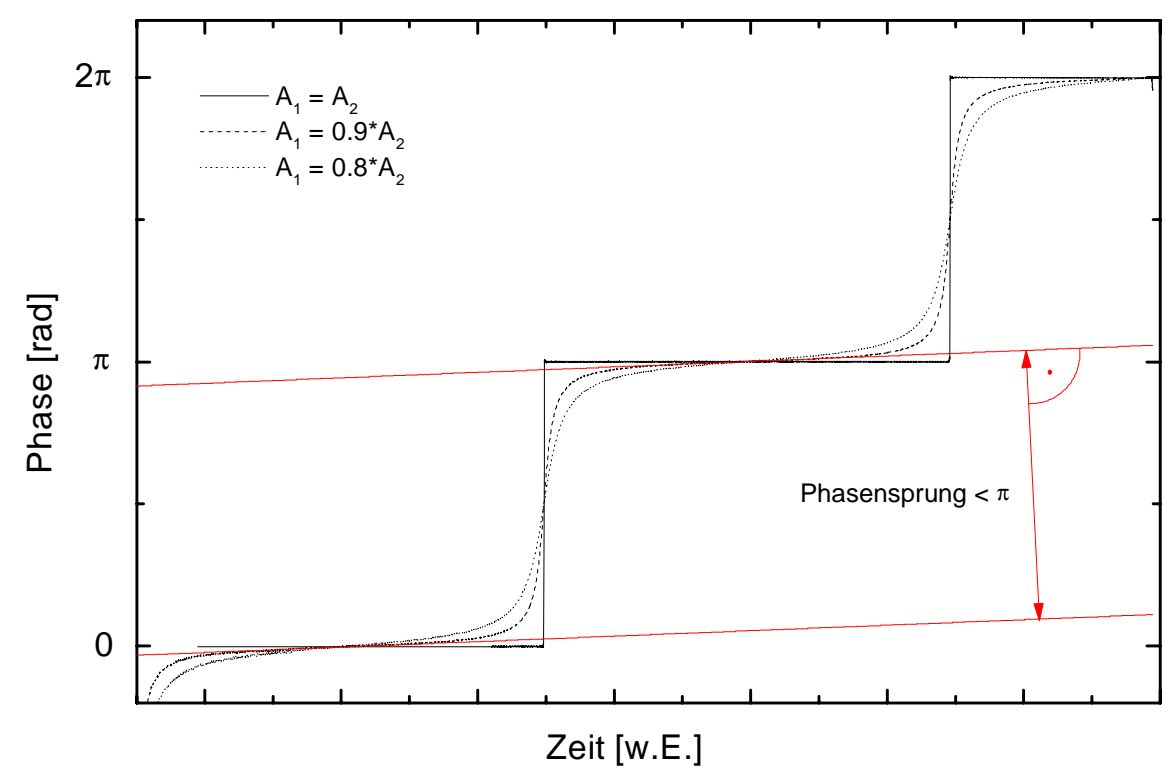

Abb. 3.4: Phasenverläufe einer Schwebung mit Frequenzen $\omega_{1}=1.02 \cdot \omega_{2}$ und Amplituden $\mathrm{A}_{1}=\mathrm{A}_{2} ; 0.9 \cdot \mathrm{A}_{2} ; 0.8 \cdot \mathrm{A}_{2}$. Mit Hilfe der Fitgeraden (grau) wird der Wert des Phasensprungs bestimmt: Für ungleiche Amplituden ist er kleiner als $\pi$.

\footnotetext{
${ }^{12}$ Die Phase sei hier erst einmal nur anschaulich durch Abb. 3.3 bzw. Gl. 3.2 eingeführt. Die Beschreibung ihrer Berechnung erfolgt in Kap 3.4.2.
} 
Berücksichtigung der Phasensprünge ist dann durch $\left(\omega_{1}+\omega_{2}\right) / 2$ gegeben. Sind die Amplituden ungleich, werden die Phasensprünge weicher und vom Betrag kleiner als $\pi$. Die Richtung des Phasensprungs, ob nach oben oder unten, wird von der Phase mit der stärkeren Amplitude bestimmt (s. Abb. 3.4). Bei der mittleren Steigung der Phase geht das Amplitudenverhältnis der beiden Schwingungen ein: für eine verschwindende Amplitude $A_{2}$ konvergiert die mittlere Steigung gegen $\omega_{1}$. Die Phase ist somit ein empfindlicher Indikator für das Amplitudenverhältnis der beiden Schwinger. 


\subsection{Erzeugung kurzer Lichtpulse und spektrale Filterung}

Die verwendeten ultrakurzen Pulse wurden mit einem Titan-Saphirlaser (Repetitionsrate $79,79 \mathrm{MHz}$, mittlere Leistung bis $500 \mathrm{~mW}$ ) erzeugt und in einem separaten Aufbau mit Standardtechniken (kollineare und nichtkollinieare SHG [54]) charakterisiert. Mit Hilfe einer Dispersionskompensationsstrecke [55] können die Chirpparameter (s. Gl. 3.2) minimiert werden $\left(|\alpha|<1.5 \cdot 10-4 \mathrm{fs}^{-2}\right.$ und $|\beta|<5.5 \cdot 10-6 \mathrm{fs}^{-3}$ ). Typische Pulse sind nahezu gaußförmig mit einer Dauer (= mittlere Halbwertsbreite der Intensität) von 30 bis 50 fs.

Es wurden zwei Methoden zur spektralen Filterung der Pulse eingesetzt: Eine Prismenstrecke (s. Abb. 3.5), die sich von der Dispersionskompensationsstrecke nur durch einen zusätzlichen Spalt unterscheidet, und ein Pulsformer [56] (s. Abb. 3.7). In der experimentellen Realisierung liegt der Vorteil der Prismenstrecke in ihrer besseren Unterdrückung der Satellitenpulse ${ }^{13}$ (relative Intensität der Satellitenpulse $\sim 10^{-6}$ ), während mit dem Pulsformer spektral schmalere Pulse erzeugt werden können.

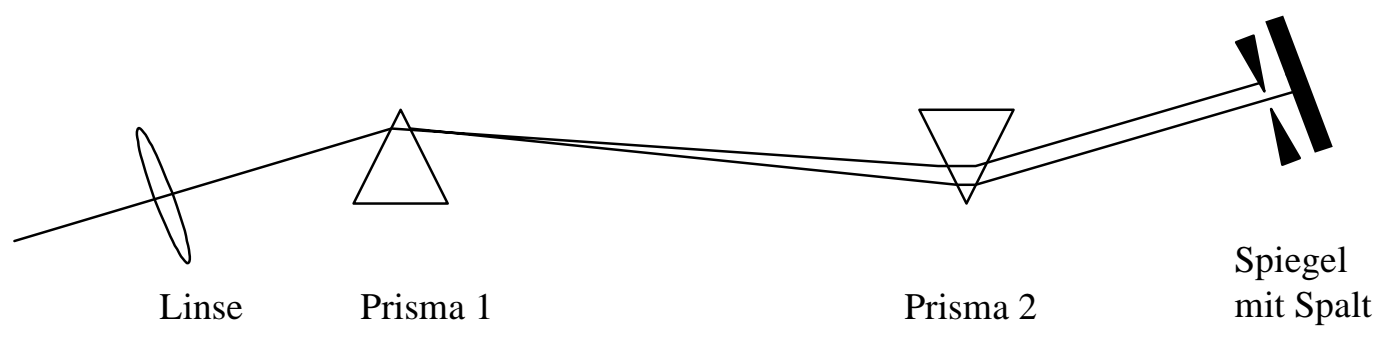

Abb. 3.5 : Dispersionskompensationsstrecke mit Spalt in der Fokalebene der Linse zur spektralen Filterung

\footnotetext{
${ }^{13}$ Satellitenpulse $=$ zusätzliche Modulationen in den Flanken des eigentlichen Pulses.
} 


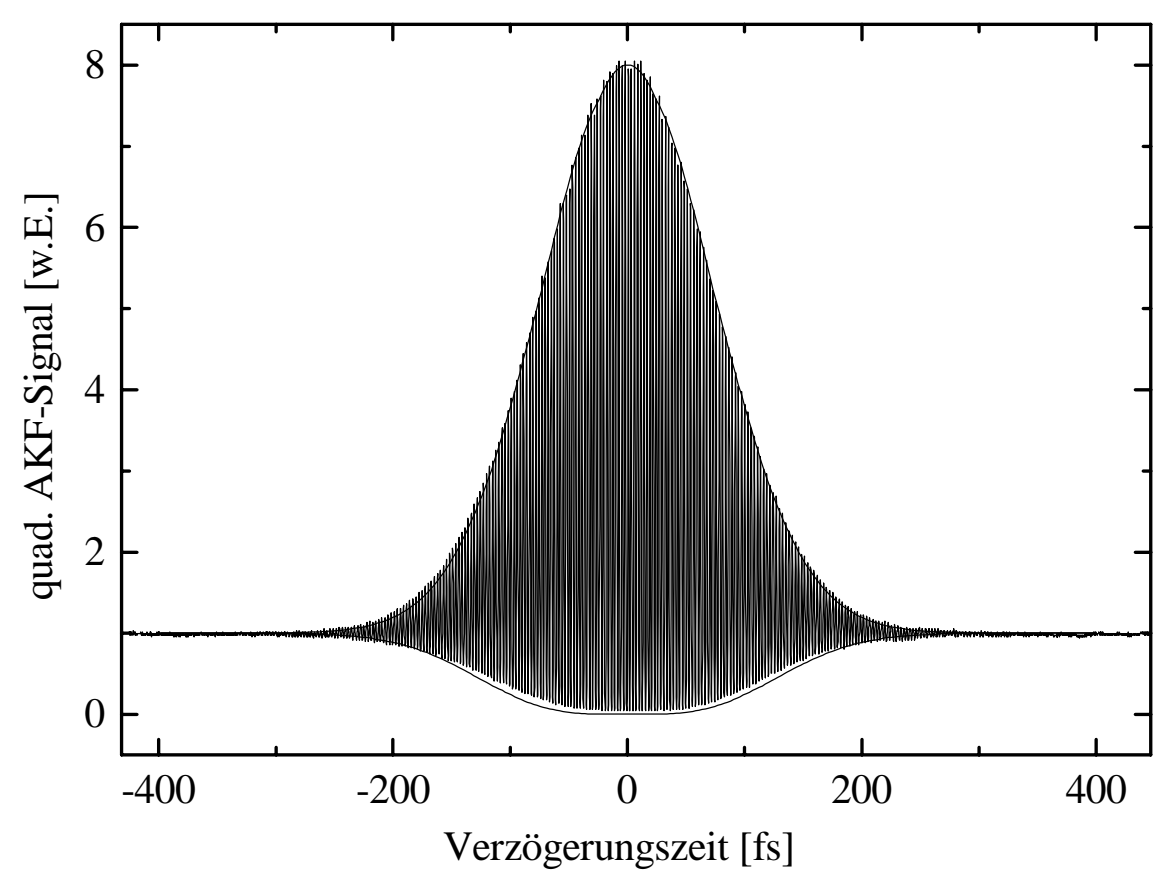

Abb. 3.6 : Quadratische Autokorrelationsspur der spektral gefilterten Pulse mit voller spektralen Halbwertsbreite in der Intensität von $21 \mathrm{meV}$ und Einhüllende eines simulierten, chirpfreien Pulses mit voller zeitlicher Halbwertsbreite in der Intensität von 80 fs.

Wegen der hohen Anforderungen an die Qualität der Pulse hinsichtlich eines gaußförmigen Verlaufes der Flanken des Pulses werden die Testpulse mit der Prismenstrecke gefiltert, wohingegen die Pumppulse mit dem Pulsformer erzeugt werden, da sie spektral möglichst schmal sein sollen.

Die Prismenstrecke besteht aus zwei Prismen (Quarz, Schnittwinkel 66,9², zur Unterdrückung der Reflexion im Brewsterwinkel orientiert), einer Linse (Brennweite $50 \mathrm{~cm}$ ) und einem Spiegel mit einem Spalt, der in die Fokalebene der Linse eingebracht wird. Dadurch erhält man die Möglichkeit, in der Spaltebene das Spektrum räumlich zu filtern. Durch die langbrennweitige Linse werden eine spektral scharfe Filterkante und die damit verbundenen Oszillationen im Zeitbereich vermieden: Ihr gaußförmiger Fokusdurchmesser gewährleistet eine entsprechend weiche Abschneidefunktion. Durch Verschieben der Prismen kann der lineare Chirp kompensiert werden. Eine typische quadratische Autokorrelation eines so erzeugten $80 \mathrm{fs}$ Puls ist in Abbildung 3.6 aufgetragen.

Ein Pulsformer ist im Prinzip ein Fourierfilter, bestehend aus einem Gitter (1200 Linien/mm), einer Linse (Brennweite $25 \mathrm{~cm}$ ) und einem Spiegel mit einer Maske, mit dem das Amplitudenspektrum wie auch das Phasenspektrum der Laserpulse kontrolliert werden kann. Die spektralen Komponenten werden in der Fokalebene 


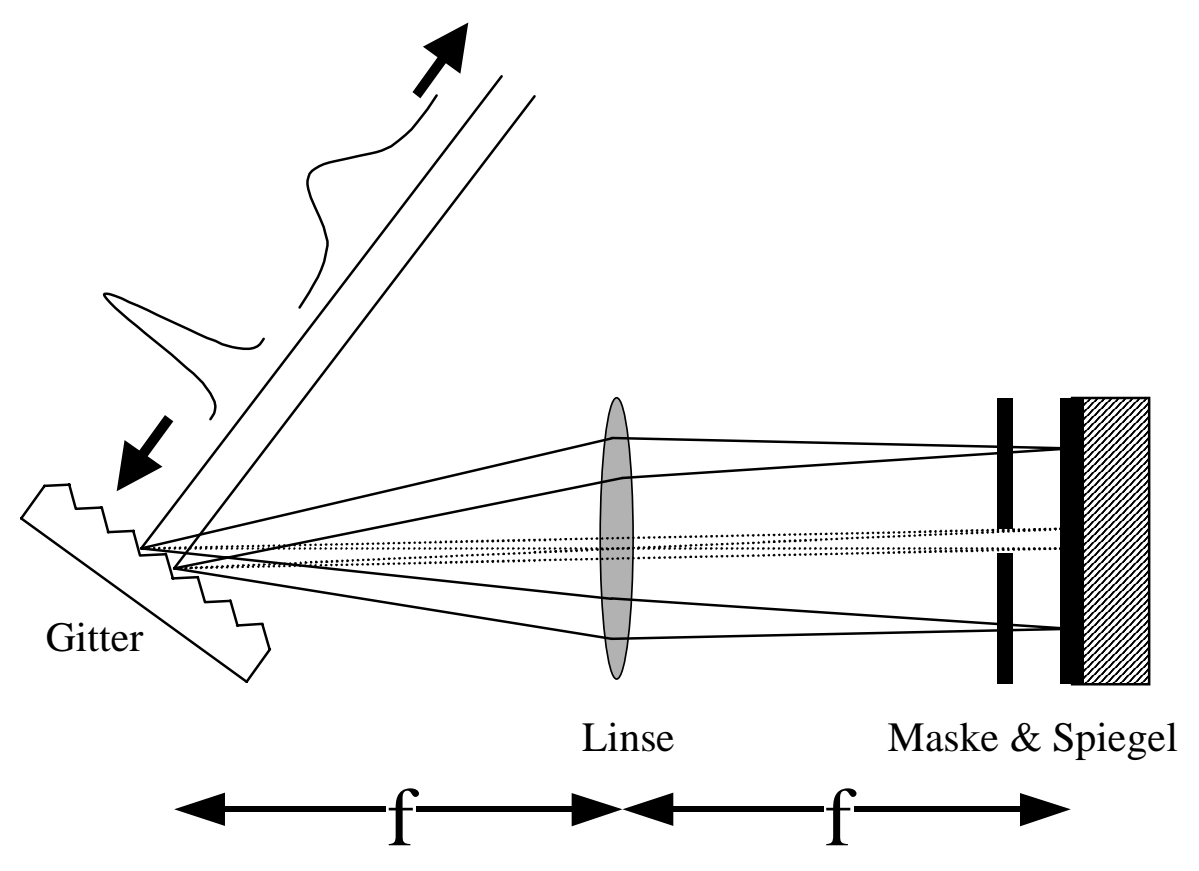

Abb. 3.7: Schematischer Aufbau eines Pulsformers

räumlich mit einer geeigneten idealerweise gaußförmigen Maske gefiltert. Die spektralen Komponenten des reflektierten, rückläufigen Strahls werden über die Linse und das Gitter wieder vereinigt.

In den durchgeführten Experimenten sollten inkohärente Ladungsträger bestimmter Energie mit dem Pumppuls zeitlich sehr viel früher (ca. $10 \mathrm{ps)}$ vor dem Testpuls generiert werden. Der genaue Generierungsprozess der Ladungsträger und damit die genaue zeitliche Form der Pumppulses spielte keine Rolle. Ein einfacher Spalt reichte deshalb als Maske aus. Durch leichtes Verschieben des Spaltes aus der Brennebene der Linse konnten zusätzlich scharfen Abschneidekanten minimiert werden. Mit dem Pulsformer werden aus den Titan-Saphir-Laserpulsen Pumppulse mit einer spektralen Breite von $\Delta \lambda=1,6 \mathrm{~nm}$ mit Zentralwellenlängen im Bereich von 765 bis $840 \mathrm{~nm}$ erzeugt (s. Abb. 3.8). Die so erzeugten Pulse haben eine zeitliche Dauer von rund 0,5 ps. 


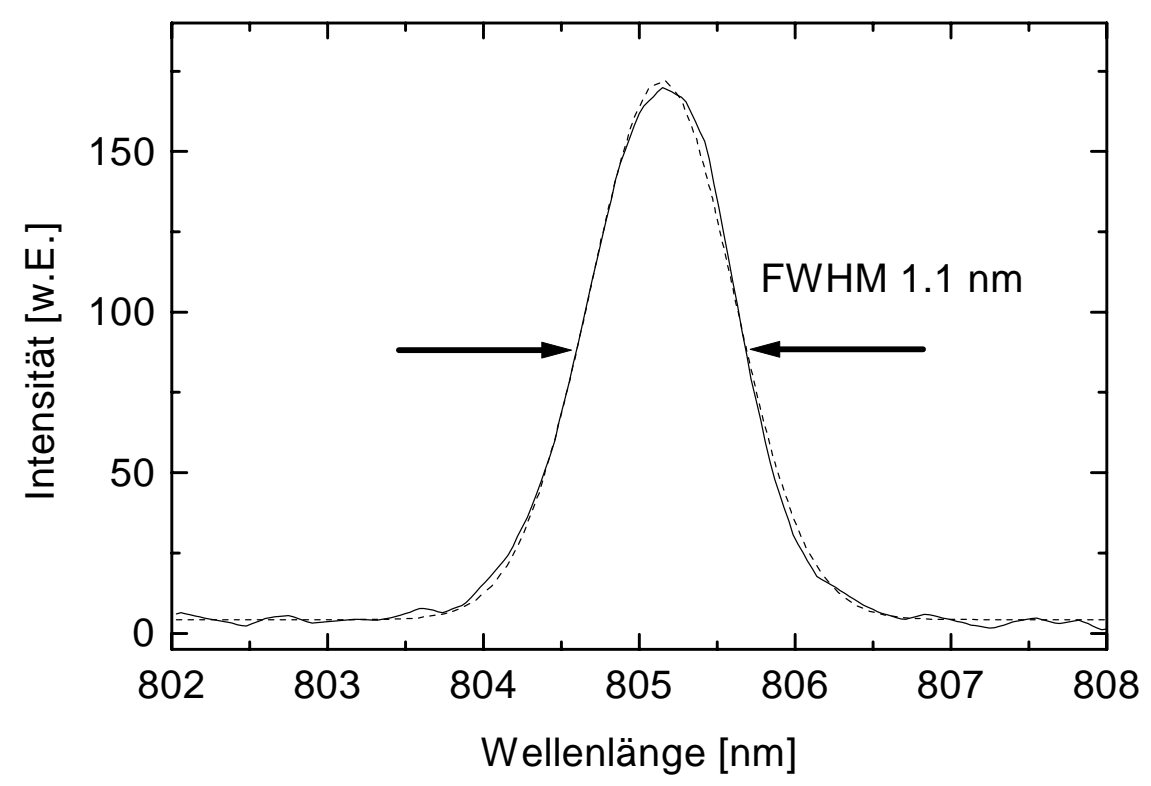

Abb. 3.8: Spektrum eines mit dem Pulsformer erzeugten Pumppulses (Fluktuationen in den Flanken sind Rauschen der CCD-Zeile). Gestrichelt: Gaußkurve mit FWHM =1.1 nm.

\subsection{Methode der interferometrischen Kreuzkorrelation}

Im Rahmen dieser Doktorarbeit wurde die Methode der interferometrischen Kreuzkorrelation zur Messung von Amplitude und Phase eines elektrischen Lichtfeldes weiterentwickelt. Zwar wurden die zugrunde liegenden Konzepte schon vor langer Zeit in der Fourierspektrokopie entwickelt [57], doch wurden ihre Vorzüge der interferometrischen Kreuzkorrelation, insbesondere ihre große Dynamik noch nicht in den Bereich der fs-Spektroskopie übertragen. Die derzeitig realisierte Apparatur erreicht eine Dynamik in der Amplitude des Signalpulses innerhalb einer Messung von 3.5 Größenordnungen, welches andere Amplituden und Phasen messende Techniken im Feld [58-61 und Ref. darin] um 1 bis 1.5 Größenordnungen übertrifft.

Die Methode besteht einerseits aus dem optisch-mechanischen Aufbau und zum anderen aus dem dazugehörigen numerischen Auswertungsalgorithmus [62]. Drei Signale werden simultan aufgezeichnet: a) eine Kreuzkorrelation der Signalpulse mit den Referenzpulsen, deren Signal proportional zur optischen Antwort der Probe ist, b) eine Autokorrelation der Referenzpulse, die die Zentralfrequenz der verwendeten Laserpulse überprüft und c) einer Autokorrelation eines cw-HeNe Lasers, mit deren 
Hilfe die Zeitachse kalibriert wird. Die HeNe-Autokorrelation ist im wesentlichen eine Phasenmessung, mit der sämtliche Längenschwankungen der Interferometerarme aufgrund thermischer Fluktuationen oder mechanischer Instabilitäten aufgezeichnet werden. Längenschwankungen können so anschließend herausgerechnet werden.

\subsubsection{Experimenteller Aufbau}

Die Veränderung des Interferenzmusters wird mit zwei rauscharmen Siliziumphotodioden, die jeweils im Zentrum der beiden Interferenzmuster hinter einer Lochblende positioniert sind, als Funktion der Verzögerungszeit (relative Längenänderung der Interferometerarme) gemessen (s. Abb. 3.9). Durch Subtraktion der beiden Signale und der Verwendung eines räumlichen Filters werden Intensitätsfluktuationen und Richtungsschwankungen des Lasers minimiert. Die Verzögerungstrecke besteht aus zwei Spiegeln und einer Präzisionstranslationsstrecke, die über einen 1:20 Übersetzungshebel von einer mit einem Servomotor getriebenen Mikrometerschraube bewegt wird. Zur Schwingungsentkopplung zwischen Motor und interferometrischem Aufbau wird ein breites Gummiband verwendet. Die Strahlteiler (BK7) sind leicht keilförmig (Winkel 1.5), um Mehrfachreflexionen zu vermeiden. Für ein qualitativ gutes Interferenzmuster und damit verbunden für ein geringeres Rauschen wird die genaue Überlagerung der beiden Lichtstrahlen mit einer CCDKamera und einer Irisblende kontrolliert. Der Akzeptanzwinkel für den eingekoppelten Laserstrahl (typischer Strahlparameter ${ }^{14} 1 \mathrm{~mm}$ ) wird durch eine Irisblende vor und hinter dem Interferometer (Länge der Interferometerarme $\cong$ Abstand der beiden Blenden $\cong 1 \mathrm{~m}$ ) auf unter $1 \mathrm{mrad}$ festgelegt. Der Kegelwinkel des auf die Probe einfallenden Lichtbündels beträgt typischerweise $12 \mathrm{mrad}$. Damit Längenfluktuationen der Interferometerarme rechnerisch eliminiert werden können, ist es für eine hohe Phasenauflösung wichtig, daß die drei Strahlen den gleichen optischen Weg über die gleichen optischen Komponenten durchlaufen: Die Kreuzkorrelation wird 1 $\mathrm{cm}$ über den beiden Autokorrelationen geführt, die ihrerseits ineinander laufend mittels chromatischen Filtern vor den Detektoren getrennt werden können. Linsen, die für die Fokussierung und das Sammeln des Lichtes an der Probe notwendig sind, werden ebenfalls im Referenzarm eingebracht (alle aus BK7 mit Brennweite $8 \mathrm{~cm}$ ). Luftturbulenzen werden durch einen Schutzkasten um den gesamten interferometrischen Aufbau reduziert.

\footnotetext{
${ }^{14}$ Der Strahlparameter a beschreibt die Breite eines Lichtstrahles und ist bei gaußförmigen Lichtbündeln gegeben durch $I(r)=I_{0} \cdot \exp \left(-r^{2} / a^{2}\right)$.
} 


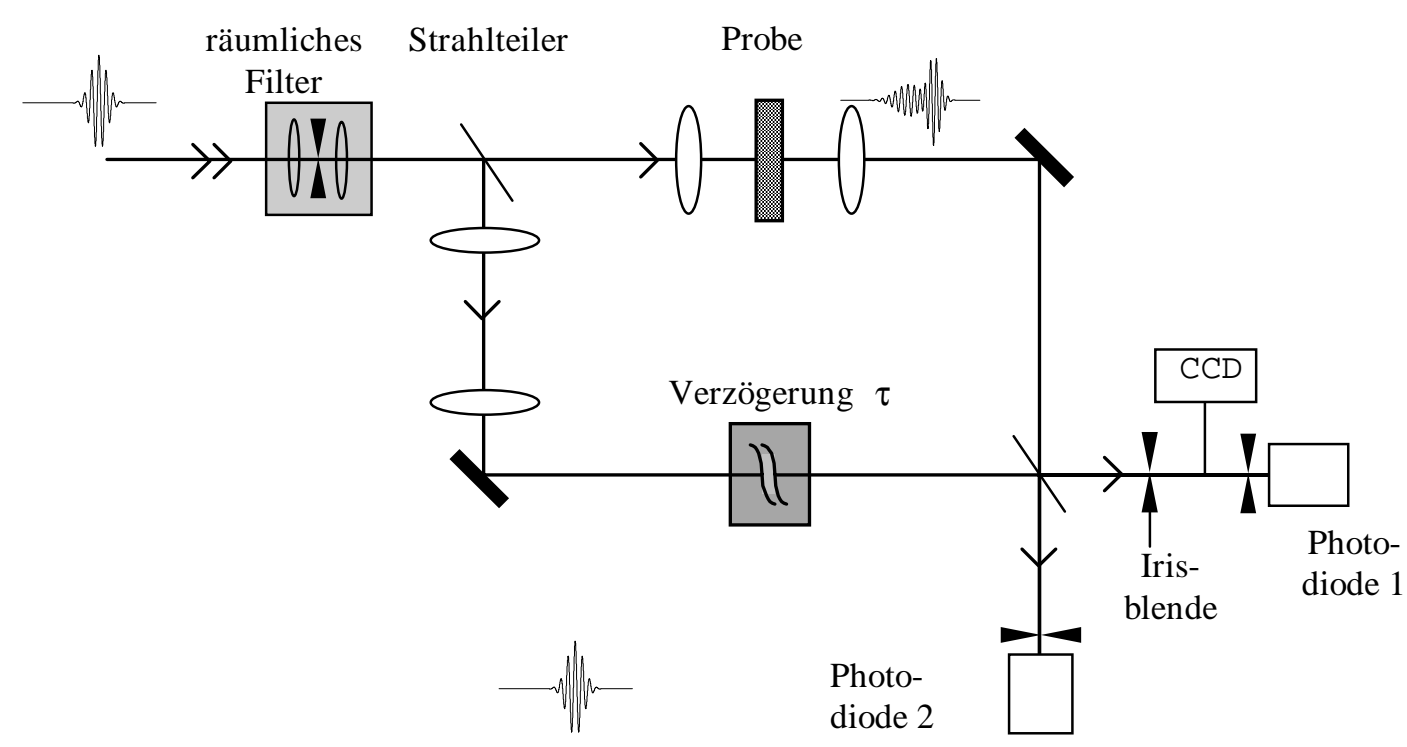

Abb. 3.9: Experimenteller Aufbau. Bis auf die Probe sind die optischen Wege der drei Stahlengänge (Kreuzkorrelation, Ti:Saphir- und HeNe-Autokorrelation) gleich.

Da Dispersion in jedem Interferometer zu erheblichen Artefakten führen kann ${ }^{15}$, ist es wichtig, die Dispersion des Interferometers zu überprüfen. Dieses wird mittels eines Spektrometers hinter dem leeren Interferometer erreicht, welches die spektrale Phase mißt und eine lineare Phase für alle interessierenden Frequenzen zeigen soll: Verstimmung der Verzögerung soll eine affine Auf- und Abbewegung der Frequenzkomponenten bewirken. Krümmungen in der spektralen Phase werden durch einen identischen Aufbau der beiden Arme des Interferometers vermieden.

Der Aufbau wurde in der Mach-Zehnder-Geometrie mit ihren zwei räumlich getrennten Strahlteilern angefertigt. Die Michelson-Geometrie, die nur einen Strahlteiler verwendet, der doppelt für Hin- und Rückrichtung genutzt wird, ist für Transmissionsmessungen nicht geeignet, da die Probe vom Lichtstrahl nur einfach durchlaufen werden soll. Die Michelson-Geometrie bietet sich für Reflexionsmessungen an.

Die Probe wurde in einen Immersionskryostaten eingebaut, so daß sie auf 2 Kelvin abgekühlt werden konnte. Der Chirp der Laserpulse, der durch die Kryostatenfenster (4 Fenstern BK7, 2 aus Duran, alle 2 mm dick) aufgeprägt wird, wird durch ent-

\footnotetext{
${ }^{15}$ Dispersion eines Interferometers führt einerseits zum Verlust von Zeitauflösung. Unterschiedliche Dispersion der beiden Interferometerarme kann anderseits zu Fehlern bei der Bestimmung der Antwortfunktion der Probe führen.
} 
sprechendes Glas im Referenzarm abgeglichen. Um bandbreitenbegrenzte Pulse auch auf der Probe zu erzielen, welches wichtig für Experimente im nichtlinearen Regime ist, wird der durch die Kryostatenfenster induzierte Chirp vor dem Interferometer mit einer Prismen-Dispersionsstrecke überkompensiert .

\subsubsection{Numerischer Auswertealgorithmus}

Im Interferometer werden die Signalpulse $\mathrm{E}_{\text {sig }}(\mathrm{t})$ mit den um die Zeit $\tau$ verzögerten Referenzpulsen $E_{\text {ref }}(t)$ überlagert. Das gemessene Signal $I(\tau)$ der Photodioden ist die Summe der Einzelintensitäten und einem Interferenzterm $\Delta \mathrm{I}(\tau)$, der Kreuzkorrelation.

$$
\mathrm{I}(\tau) \propto \mathrm{E}_{\mathrm{sig}}^{2}+\mathrm{E}_{\mathrm{ref}}^{2}+\Delta \mathrm{I}(\tau)
$$

mit

$$
\Delta \mathrm{I}(\tau)=2 \cdot \int_{\substack{\text { Detektor } \\ \text { Zeitkonstante }}} \mathrm{dt} \mathrm{E}_{\text {sig }}(\mathrm{t}) \cdot \mathrm{E}_{\text {ref }}(\mathrm{t}+\tau)
$$

Durch Abziehen der Analogsignale der Photodioden ${ }^{16}$ heben sich die ersten zwei Terme auf der rechten Seite in Gl. 3.4 weg, die keinerlei Informationen erhalten, sondern nur zum Rauschen beitragen. Basisschwankungen aufgrund von Intensitätsfluktuationen des Lasers werden so vermieden. Da die beiden Interferenzmuster komplementär zueinander stehen und sich um den Phasenwinkel von $180^{\circ}$ unterscheiden $^{17}$, bleibt der dritte Term übrig. Damit ist das gemessene Signal der beiden subtraktiv geschalteten Photodioden proportional zum Kreuzkorrelationssignal $\Delta \mathrm{I}(\tau)$. Das gemessene Signal ist im wesentlichen die über den Referenzpuls gemittelte Probenantwort:

$$
\Delta \mathrm{I}(\tau) \propto \int_{\substack{\text { Detektor } \\ \text { Zeitkonstante }}} \mathrm{dt} \mathrm{E}_{\text {ref }}(\mathrm{t}) \cdot \mathrm{E}_{\text {sig }}(\mathrm{t}+\tau)=\left\langle\mathrm{E}_{\text {sig }}(\tau)\right\rangle_{\substack{\text { gemittelt uber } \\ \text { Referenzpuls }}}
$$

\footnotetext{
${ }^{16}$ Die Photodioden wurden parallel mit entgegengesetzter Polung geschaltet.

${ }^{17}$ Der Unterschied in der Phase kommt durch den Phasensprung bei der Reflexion am Strahlteiler zustande.
} 
Die Amplitude $\mathrm{I}_{0}(\tau)$ und die Phase $\varphi(\tau)$ des Interferenztermes $\Delta \mathrm{I}(\tau)$ kann durch Berechnung des Integrals

$$
\frac{\omega_{\mathrm{L}}}{2 \pi} \cdot \int_{\tau-\pi / \omega_{\mathrm{L}}}^{\tau+\pi / \omega_{\mathrm{L}}} \mathrm{d} \tau^{\prime} \Delta \mathrm{I}\left(\tau^{\prime}\right) \exp \left(-\mathrm{i} \omega_{\mathrm{L}} \tau^{\prime}\right) \cong \frac{1}{2} \mathrm{I}_{0}(\tau) \cdot \mathrm{e}^{\mathrm{i} \varphi(\tau)}
$$

bestimmt werden, wobei $\omega_{\mathrm{L}}$ die Trägerfrequenz der Pulse ist. Die Methode ist identisch mit denjenigen von Fourier-Spektrometern, und die Vorgehensweise entspricht einem numerischen Lock-In-Verstärker Verfahren.

Die Berechnung der Amplitude, der Phase und der Zeitskalierung werden mit einem Computer durchgeführt. Pro Schwingung, deren Periodenlänge bei dem verwendeten Licht mit Wellenlänge $\lambda \approx 800 \mathrm{~nm}$ rund $2.7 \mathrm{fs}$ beträgt, werden 12-14 Meßpunkte aufgenommen. Typische Rohdaten sind in Abb. 3.10 aufgetragen. Während der Auswertung sind alle drei Signale eine Funktion der Meßpunktnummer i. Die tatsächliche Zeitverschiebung $\tau$ (in Einheiten von fs) wird nachträglich bestimmt.

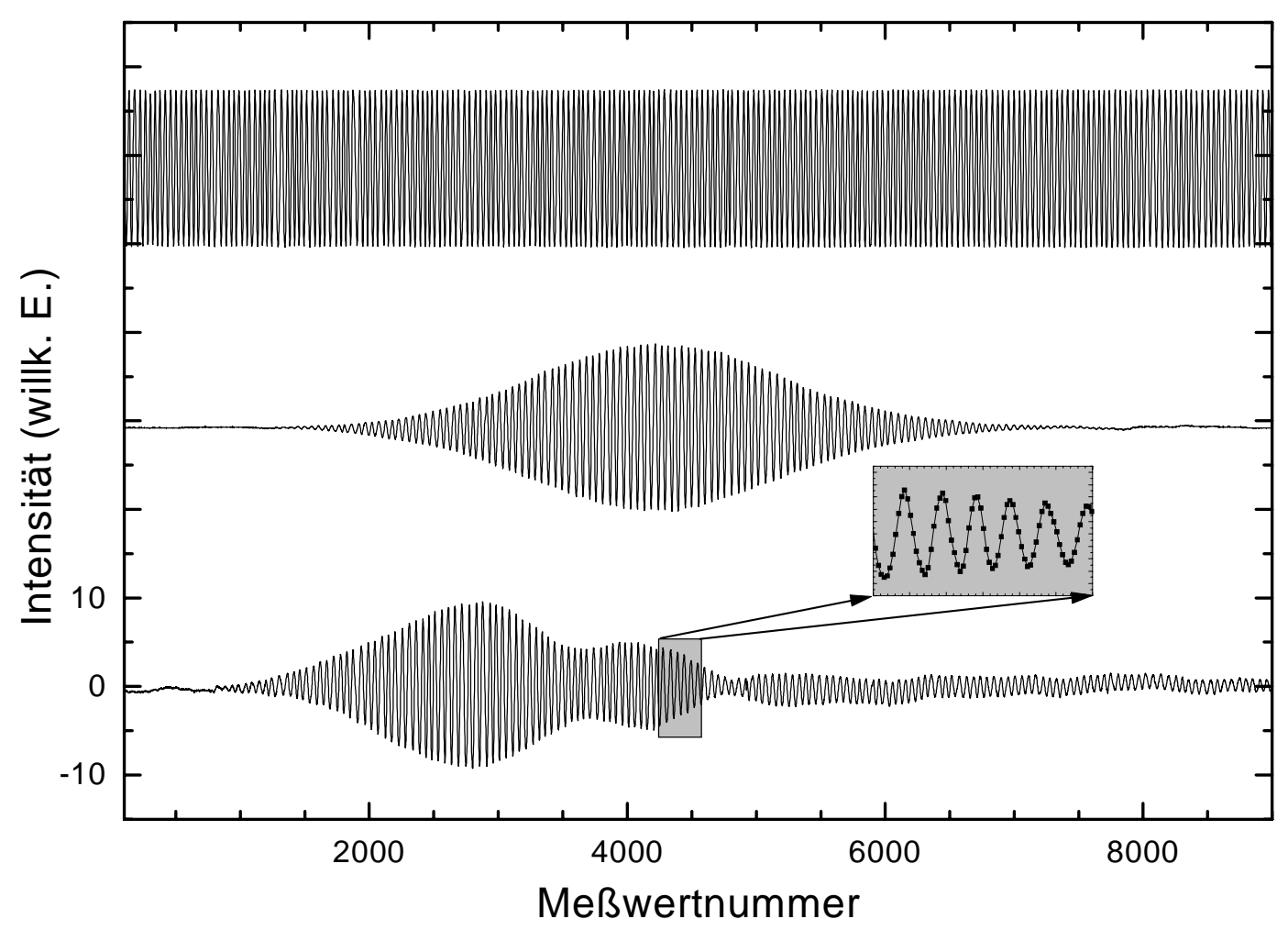

Abb. 3.10: Typische Rohdaten als Funktion der Meßpunktnummer, wie sie vom Computer aufgezeichnet werden. Von oben nach unten: HeNe-Laser Autokorrelation, Titan-Saphir Laser Autokorrelation und die Kreuzkorrelation mit einem vergrößerten Ausschnitt. 
Als erster Schritt in der Auswertung werden die diskreten Datenpunkte der cw-HeNe Autokorrelation interpoliert und ihre M Nullstellen gezählt. Zur groben, vorläufigen Bestimmung der Frequenz $v_{\mathrm{HeNe}}$ der $\mathrm{cw}-\mathrm{HeNe}-\mathrm{Laser}$ Autokorrelation in Einheiten von Schwingungen pro Meßpunktnummer wird $v_{\mathrm{HeNe}}=(\mathrm{M}-1) \cdot \pi / \mathrm{N}$ gewählt, wobei N die Gesamtanzahl der Meßpunkte ist. Durch Berechnung des Integrals Gl. 3.7 kann die Phase der cw-HeNe-Laser Autokorrelationsspur $\varphi_{\mathrm{HeNe}}(\mathrm{i})$ bestimmt werden. Sie wird mit einem Gaußfilter der Breite $1 / \mathrm{f}_{\mathrm{HeNe}}$ (eine Periodenlänge der Schwingung der HeNe-Laser Autokorrelation) geglättet. Anschließend wird die Gesamtsteigung $\Delta v_{\mathrm{HeNe}}=\left(\varphi_{\mathrm{HeNe}}(\mathrm{N})-\varphi_{\mathrm{HeNe}}(1)\right) / \mathrm{N}$ der Phase $\varphi_{\mathrm{HeNe}}(\mathrm{i})$, welche durch eine ungenau bestimmte Frequenz $v_{\mathrm{HeNe}}$ zustande kommt, behoben, indem eine neue, genauere HeNe Frequenz $v_{\mathrm{HeNe}} \rightarrow v_{\mathrm{HeNe}}+\Delta v_{\mathrm{HeNe}}$ definiert wird. Die Phase $\varphi=$ $\varphi_{\mathrm{HeNe}}(\mathrm{i})+v_{\mathrm{HeNe}} \cdot \mathrm{i}$ zeichnet sämtliche thermischen und mechanischen Schwankungen des Interferometers sowie die Verzögerung durch die Verzögerungsstrecke auf. Phasensprünge von $2 \pi$ enthalten keine physikalische Information und werden mathematisch eliminiert, indem die Phasen kontinuierlich fortgesetzt werden.

Als nächster Schritt werden die Datenpunkte der Titan-Saphir-Laser Autokorrelation interpoliert. Mit der vorläufigen Laserfrequenz $v_{\mathrm{TiSa}}=v_{\mathrm{HeNe}} \cdot \lambda_{\mathrm{HeNe}} / \lambda_{\mathrm{TiSa}}$ wird die Amplitude und Phase mit dem Integral Gl. 3.7 gefunden, wobei $\lambda_{\mathrm{H} \text { eNe }}=632,8 \mathrm{~nm}$ die Wellenlänge des HeNe und $\lambda_{\text {TiSa }}$ die ungefähre Laserzentralwellenlänge im Experiment ist $(\approx 820 \mathrm{~nm}$ ). Glätten der Amplitude und der Phase über eine Periode und Beheben der Steigung in der Phase $\Delta v_{\mathrm{TiSa}}=\left(\varphi_{\mathrm{TiSa}}(\mathrm{N})-\varphi_{\mathrm{TiSa}}(1)\right) / \mathrm{N}$ liefern eine genauere Laserfrequenz durch $v_{\text {TiSa }} \rightarrow v_{\text {TiSa }}+\Delta v_{\text {TiSa }}$. Diese Frequenz wird wiederum benutzt, um das Integral Gl. 3.7 neu zu berechnen, welches eine genaueren Amplitude und Phase liefert. Diese Prozedur wird wiederholt bis $v_{\text {TiSa }}$ gegen einen festen Wert konvergiert.

Schließlich werden die Datenpunkte der Kreuzkorrelation interpoliert, und die Phase und Amplitude werden unter Verwendung von $v_{\text {TiSa }}$ berechnet. Die driftkorrigierte Phase der Kreuzkorrelation ist $\varphi_{\mathrm{CC}}(\mathrm{i}) \rightarrow \varphi_{\mathrm{CC}}(\mathrm{i})-v_{\mathrm{TiSa}} / \nu_{\mathrm{HeNe}} \cdot \varphi_{\mathrm{HeNe}}(\mathrm{i})$.

Die Zeitskalierung wird durch $\tau(\mathrm{i})=\left(\nu_{\mathrm{HeNe}} \cdot \mathrm{i}+\varphi_{\mathrm{HeNe}}(\mathrm{i})\right) / \omega_{\mathrm{HeNe}}$ bestimmt, wobei $\omega_{\mathrm{HeNe}}=\mathrm{c}_{\mathrm{air}} \cdot 2 \pi / \lambda_{\mathrm{HeNe}}$ die Frequenz des HeNe-Lasers und $\mathrm{c}_{\text {Luft }}$ die Lichtgeschwindigkeit in Luft ist. Der Zeitnullpunkt wird in das Maximum der Titan-Saphir-Laser Autokorrelation gelegt. Die Phase wird relativ zu einer gegebenen, dem System angepaßten Frequenz wie z.B. der Resonanzfrequenz des 1s Exzitons ausgegeben. 


\subsubsection{Charakterisierung der Methode und Testmessungen}

Der mechanische Aufbau muß charakterisiert werden und der Auswertealgorithmus hinsichtlich seiner numerischen Stabilität und Zuverlässigkeit geprüft werden.

Das Einspeisen simulierter Daten in die Auswertung zeigt, daß der Algorithmus die Zentralwellenlänge der Pulse auf $0,01 \mathrm{~nm}$ genau findet und eine Dynamik über 8 Größenordnungen in der berechneten Intensität erreicht. Die Phase wird auf 1/1000 der Wellenlänge genau bestimmt. Damit liegen die Fehlerquellen der Methode weniger auf der Seite des Algorithmus als auf der Seite des Detektors bzw. des mechanischen Aufbaus.

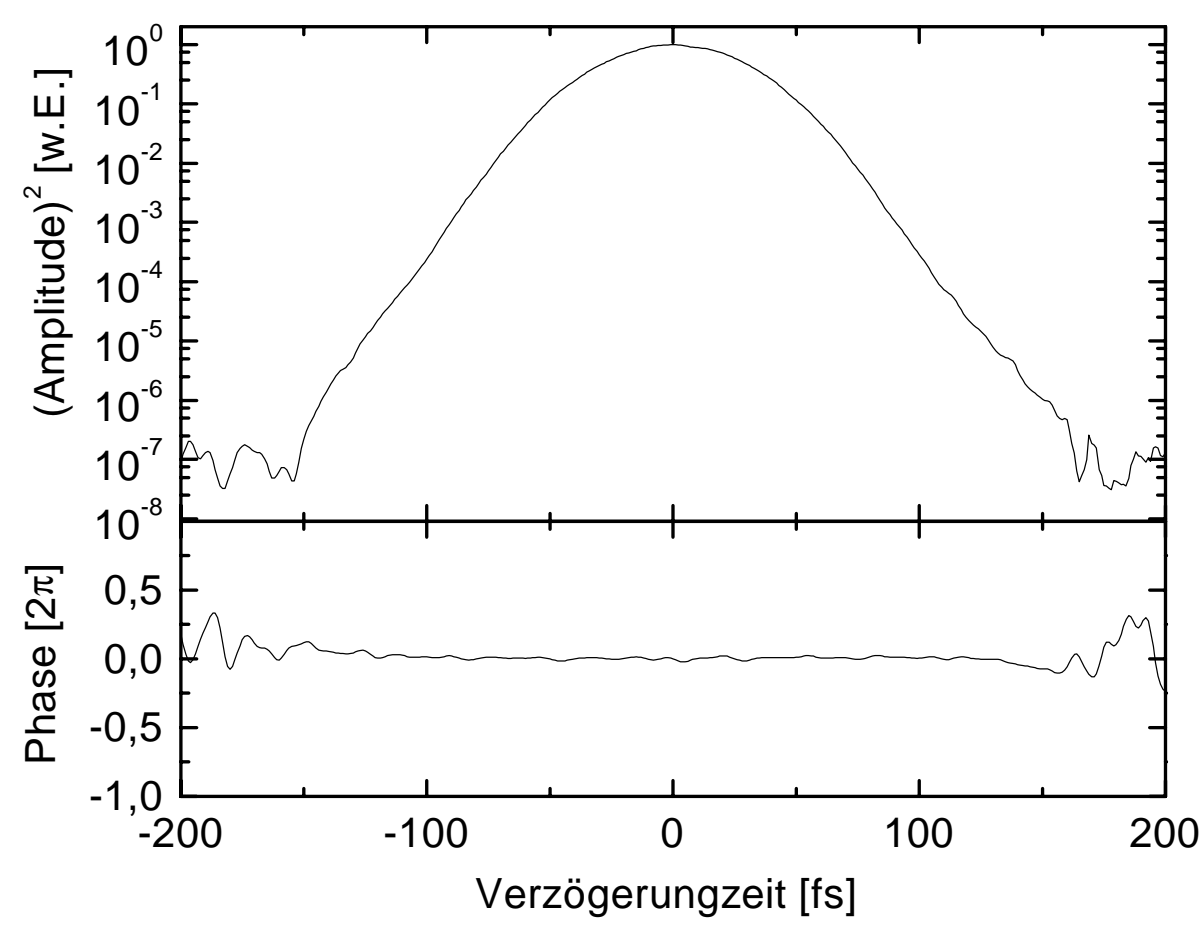

Abb. 3.11: Amplitudenquadrat und Phase der Autokorrelation der Titan-Saphir Laserpulse.

Ein Meßlauf über einen Bereich von 2 ps Zeitverzögerung dauert ungefähr 1 Minute. Messungen mit Pulsen aus dem Titan-Saphir Laser $(\lambda \approx 800 \mathrm{~nm})$ zeigen die für Autokorrelationen notwendige Symmetrie in der Amplitude und in der Phase (s. Abb. 3.11). Die Standardabweichung der Phase liegt unter 1/100 der Wellenlänge, und die Dynamik der Amplitude innerhalb eines Meßlaufes beträgt 3.5 


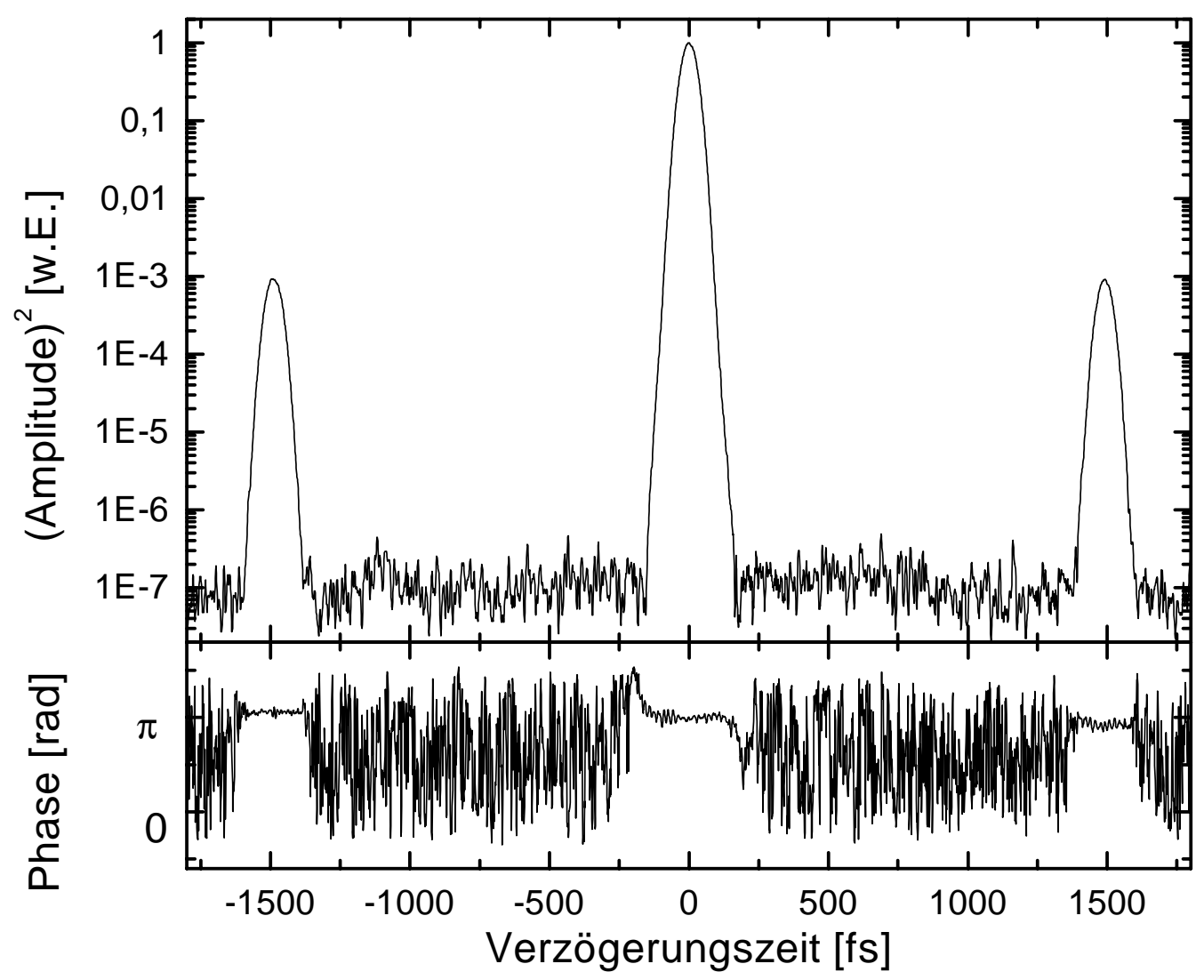

Abb. 3.12: Testmessungen an einer $0.1 \mathrm{~mm}$ dicken teilweise entspiegelten Glasplatte. Quadrierte Amplitude und Phase. Abweichungen der Phase von der Spiegelsymmetrie treten für relative Intensitäten kleiner als $10^{-6}$ auf.

Größenordnungen. Es wurden Signale von einigen $\mathrm{mW}$ bis hinunter zu $10 \mathrm{fW}$ (Signal-zu-Rausch-Verhältnis von 1) gemessen. Die geringste noch detektierbare Intensität wird durch das Detektorrauschen (Bandbreite: $30 \mathrm{~Hz}$, NEP der Photodioden: $\left.1,4 \cdot 10^{-13} \mathrm{~W}\right)$ begrenzt. Die Dynamik für höhere Energien ist durch mechanische Instabilitäten, nicht optimale Qualität des Interferenzmusters, Luftturbulenzen sowie durch den verwendeten A/D-Wandler (12 bit) begrenzt. Das Rauschen umfaßt nicht nur die Phase (Spiegelwackeln), sondern erstreckt sich auch auf die Amplitude. Abweichungen der Autokorrelation in der halblogarithmischen Auftragung von einer parabolischen Form weisen darauf hin, daß die Titan-Saphir Laserpulse nicht perfekt gaußförmig sind.

Wird hinter (oder vor) dem Interferometer eine planparallele Glasplatte (Dicke: 0.1 $\mathrm{mm}$ ) eingebracht, so daß Mehrfachreflexionen erzeugt werden, weist die Autokorrelationsspur Satellitenpulse auf (s. Abb. 3.12), die erwartungsgemäß zeitlich symmetrisch mit gleicher Intensität und symmetrischer Phase (bis hinrunter zu relativen Intensitäten von $10^{-6}$ ) liegen ${ }^{18}$.

\footnotetext{
${ }^{18}$ Ihre relative Intensität beträgt aufgrund der Entspiegelung der Glasplatte weniger als $4 \%$.
} 
Zur Demonstration der Methode wird das transmittierte Feld durch einen Interferenzfilter (Spezifikation: Zentralwellenlänge: $830 \pm 2 \mathrm{~nm}$, spektrale Breite: $10 \pm 2 \mathrm{~nm}$ ) ausgemessen (s. Abb. 3.13). Die ursprünglich um $820 \mathrm{~nm}$ zentrierten 40 fs Gaußpulse werden in eine Kaskade von Pulsen mit kleiner werdenden Amplituden aufgebrochen. Die Periodizität von $\approx 435$ fs entspricht einer spektralen Filterbreite von $10,56 \mathrm{~nm}$. Aus der Phase um $\tau=0$ kann die Zentralwellenlänge des Filters zu 828 $\mathrm{nm}$ bestimmt werden. Das Hintergrundrauschen erkennt man für frühere Zeiten $\tau<$ 250 fs bei $10^{-7}$ im Quadrat der Amplitude, die Standardabweichung der Phase ist kleiner als 1/100 Ring. Die Minima für Verzögerungszeiten zwischen 0 und 1 ps werden begleitet von Phasensprüngen, die nicht exakt $\pi$ sind und ansteigen. Ursache hierfür ist die Verstimmung des Laserpulses relativ zum Filter und das daraus resultierende asymmetrische Transmissionsspektrum mit mehr Gewicht auf der Hochenergieseite. Die Steigung der Phase ändert sich, was einer Blauverstimmung der Momentanfrequenz von $\Delta \hbar \omega=2 \mathrm{meV}$ entspricht. Das Signal für $\tau>1$ ps mit relativer Intensität von $<10^{-5}$ wird beeinflußt durch die Ungenauigkeiten des Filters, welcher für eine Unterdrückung von $10^{-5} \mathrm{im}$ Sperrbereich spezifiziert ist.

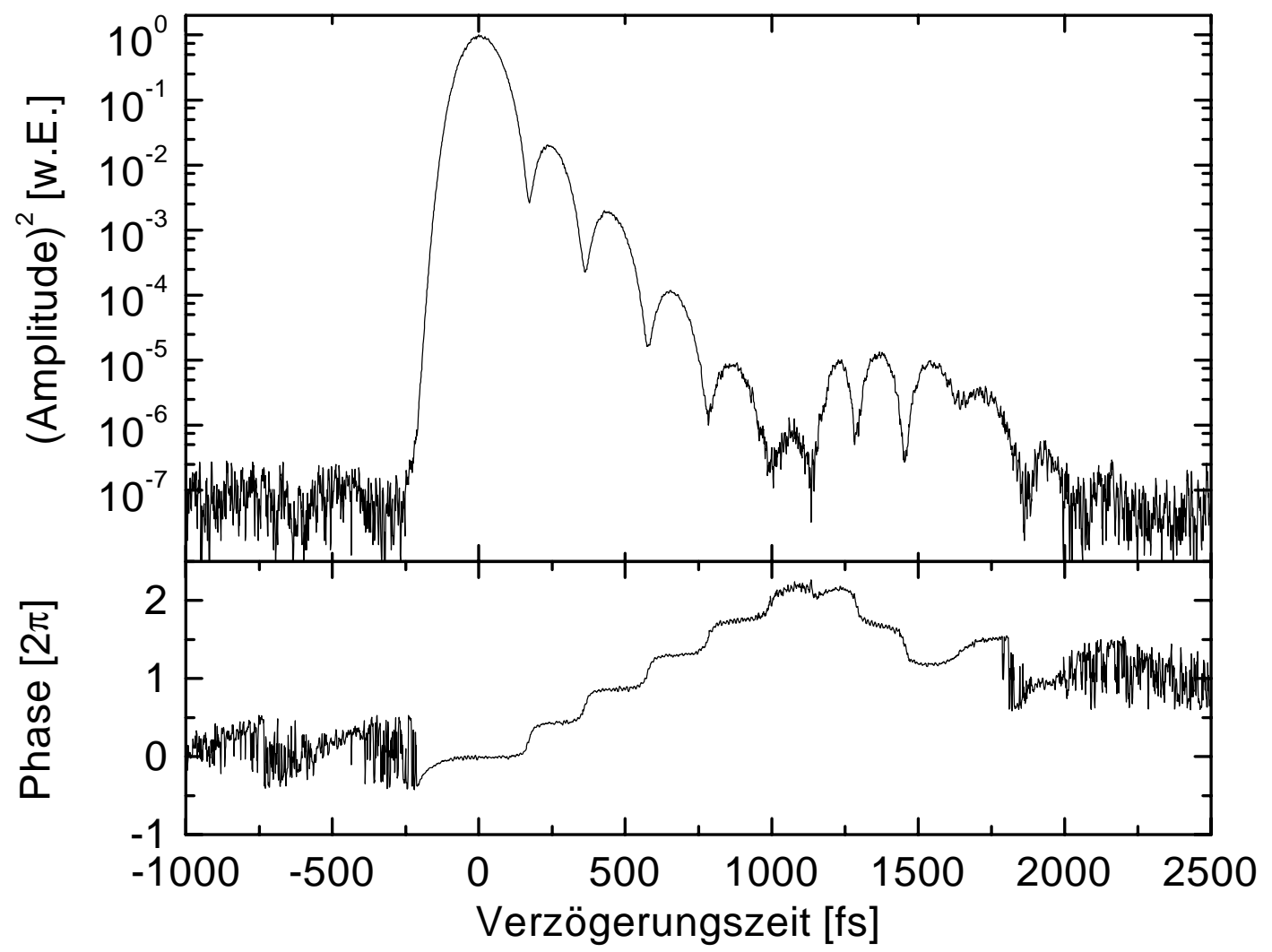

Abb. 3.13: Amplitudenquadrat und Phase der Kreuzkorrelationsspur des transmittierten E-Feldes durch einen Interferenzfilter. 


\subsection{Pump- und Testexperimente}

Neben den intensitätsabhängigen Einstrahlexperimenten wurden auch Experimente in Pump- und Test-Anordnungen durchgeführt. Dazu wurde ein zusätzlicher, stärkerer Puls unter einem externen Winkel von $45^{\circ}$ auf der Probe überlagert, der zeitlich und spektral variiert werden konnte (s. Abb. 3.14). Um kohärente Effekte zu unterdrücken [63], wird die Polarisation des Pumppulses senkrecht zu der des Testpulses gedreht. Die örtliche Überlagerung der beiden Pulse wird mittels Abbildung der Probe auf eine CCD-Kamera in Transmission des Testpulses und Betrachten der Lumineszenz des Pumppulses hergestellt und die zeitliche Verzögerung mit einem Verschiebeschlitten eingestellt. Die zeitliche Koinzidenz kann einerseits durch Betrachten des Interferenzmusters der beiden Pulse auf der Probe, anderseits durch die Transmissionsänderung auf \pm 10 fs eingestellt werden.

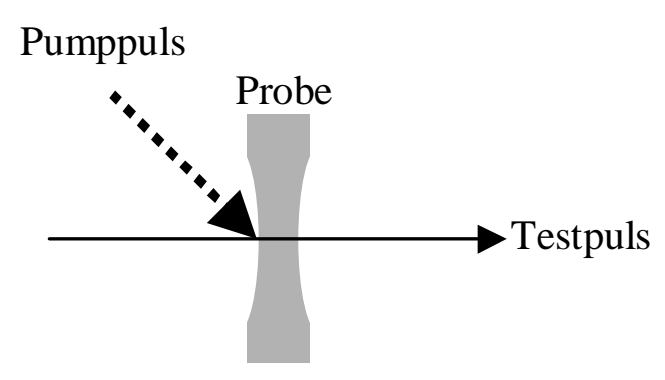

Abb. 3.14: Überlagerung von Pump- und Test-
puls im zeitlichen Abstand von $\tau$ auf die Probe 


\section{Experimentelle Untersuchungen zur Streuung von 1s-Exzitonen am ungebundenen e-h Plasma}

Wie im vorausgegangenen Kapitel gezeigt wurde, können mit der Methode der interferometrischen Kreuzkorrelation Präzisionsmessungen zur Amplitude und Phase des durch die Probe transmittierten elektrischen Feldes angefertigt werden. Es wurden Experimente zur Wechselwirkung der exzitonischen Streuzustände mit dem 1s-Exziton durchgeführt. Bei allen Experimenten war die Probe auf 2 Kelvin abgekühlt. Ergebnisse von Raumtemperaturmessungen sind unter [64, 65] zu finden.

Es werden Ergebnisse folgender Einstrahlexperimente vorgestellt (Kap. 4.1.):

- In Experimenten mit mäßigen Anregungsdichten wird die Oszillatorstärke präzise gemessen.

- Durch Variation des Spektrums der Anregungspulse wird der dominierende Streukanal bestimmt.

- Es wird die Abhängigkeit der Amplitude und Phase der Polariton-Pulspropagation von der Anregungsfluenz untersucht.

In Pump- und Test-Meßungen (Zweistrahlexperimente) wird untersucht (Kap. 4.2),

- in welcher Weise die zeitliche Dynamik der Ladungsträger eine Rolle spielt und

- in wie fern die energetische Lage der durch den Pumppuls generierten Ladungsträger in die Dämpfung eingeht.

Durch Vergleich der Meßdaten mit Simulationen werden relevante Größen wie die Oszillatorstärke und die Dämpfungskonstanten in Abhängigkeit der jeweiligen Kontrollparameter bestimmt. 


\subsection{Einstrahlexperimente zur Polariton-Pulspropagation bei niedriger und mittlerer Anregung}

Die Kontrollparameter bei den Einstrahlexperimenten sind die Intensität und das Spektrum der anregenden Laserpulse. Typische Meßdaten für Anregungen mit einem um $1.53 \mathrm{eV}$ zentrierten 40 fs Laserpuls sind in Abb. 4.1 aufgetragen. Die Phase wird relativ zur nackten Resonanzfrequenz $\hbar \omega_{0}=1,515 \mathrm{eV}$ des $1 \mathrm{~s}$-Exzitons ausgewertet.

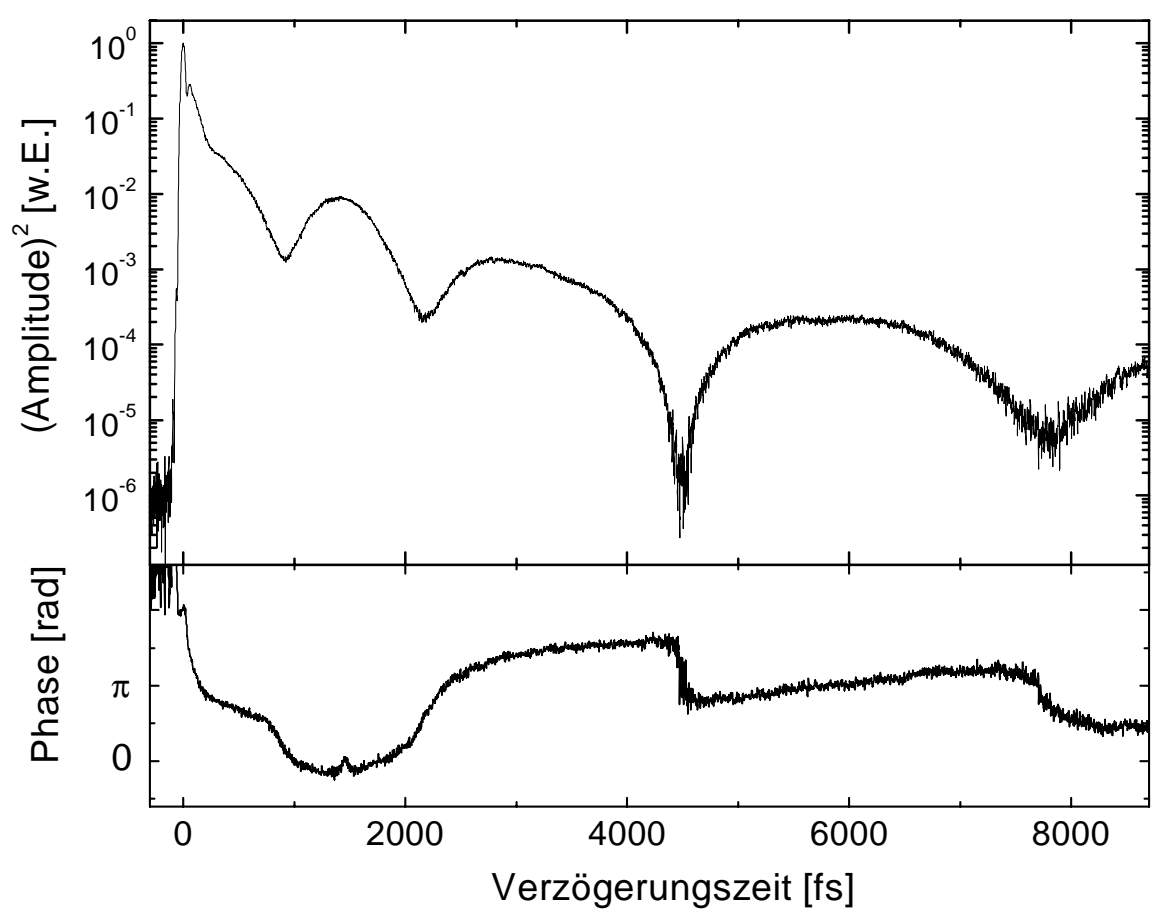

Abb. 4.1: Typische Meßergebnisse für die Amplitude (oben) bzw. die Phase (unten) des transmittierten elektrischen Feldes bei mittleren Anregungsdichten.

Man erkennt die für die Polariton-Pulspropagation charakteristischen Schwebungen mit zunehmender Periodenlänge. Die Minima in der Amplitude sind begleitet von Sprüngen in der Phase von ungefähr (aber nicht genau) $\pm \pi$, die in charakteristischer Weise abrupt oder weich bzw. nach oben oder unten gehen. Aus der veränderlichen Steigung der Phase erkennt man eine zeitlich nicht konstante Momentanfrequenz. Für $\tau<1$ ps liegt sie rund $2 \mathrm{meV}$ unterhalb der 1s-Exzitonresonanz und nähert sich dieser nichtmonoton. Das Signal ist für Zeiten > 300 fs unabhängig von der spektralen Lage der anregenden Laserpulse in einem Bereich von $\mathrm{E}_{\text {Laser }}=\hbar \omega_{\mathrm{gap}^{-}} 3$ $\mathrm{meV}$ bis $\hbar \omega_{\text {gap }}+35 \mathrm{meV}$.

Aperiodischen Schwebungen in der Intensität der Polariton-Pulspropagation wurden zum ersten Mal von Fröhlich et al. in $\mathrm{Cu}_{2} \mathrm{O}$ auf der ns-Zeitskala nachgewiesen [66]. 
Die Auflösung in der Phase konnte bisher noch nicht geleistet werden und wird im Rahmen dieser Arbeit erstmalig erbracht [67].

Die zeitliche Auflösung der Meßmethode von rund 40 fs (begrenzt durch die Breite des Referenzpulses) darf nicht darüber hinwegtäuschen, daß bei Transmissionsexperimenten durch optisch dicke Proben verschiedene Raumzeitpunkte über die Propagation miteinander verknüpft werden. Schnelle Strukturen werden durch Retardierungseffekte bei der Propagation verwischt.

Es ist zu bemerken, daß die interferometrische Kreuzkorrelation nur den kohärenten Anteil des Lichtfeldes ausmißt: Inkohärentes Licht wie beispielsweise Lumineszenz äußert sich in einem verminderten Kontrast des Interferenzmusters ${ }^{19}$ und wird durch die Korrelation unterdrückt. Da in Vorwärtsrichtung nur ein kleiner Raumwinkel innerhalb des 0 . Interferenzringes detektiert wird, unterscheidet sich diese Methode von herkömmlichen Absorptionsmessungen, die überlicherweise mit einem großen Raumwinkel arbeiten ${ }^{20}$.

\subsubsection{Bestimmung der Oszillatorstärke}

Durch Vergleich der Meßdaten mit Simulationen wird die Oszillatorstärke gewonnen. Abb. 4.2 zeigt die mit Gl. 2.23 berechneten Amplituden- und Phasenverläufe (Parameter: $\hbar \omega_{0}=1.515 \mathrm{eV}, \hbar \omega_{\mathrm{LT}} \cdot \mathrm{d}=304 \mu \mathrm{eV} \cdot \mu \mathrm{m}, 2 \hbar \gamma=170 \mu \mathrm{eV}$, $\left.\varepsilon_{\mathrm{b}}=12.55\right)$. Die Lage der Minima und der Zerfall der Amplitude wird für Zeiten $>2$ ps im wesentlichen wiedergegeben. Das Signal für frühere Zeiten ist von dem Polarisationszerfall der höheren Resonanzen bestimmt, die in diesem Modell nicht berücksichtigt werden. In der Phase werden Sprünge, nicht aber ihre korrekte Form und Richtung reproduziert. Die Lage der Minima in der Amplitude ist sensibel vom Produkt aus Kopplungsstärke und Probendicke abhängig und bietet eine präzise $\mathrm{Be}-$ stimmungsmethode der Kopplungstärke mit einem Fehler von 5\%. In der Literatur wird sie als $\hbar \omega_{\mathrm{LT}}=80 \mu \mathrm{eV}$ auf $25 \%$ genau $[68,69]$ angegeben.

Die Oszillatorstärke der 1s-Exzitonenresonanz in GaAs wird zu $\hbar \omega_{L T}=(86 \pm 5) \mu e V$ bestimmt. Sie bleibt für Anregungsdichten im Kontinuum von $10^{13} \mathrm{~cm}^{-3}$ bis $10^{15} \mathrm{~cm}^{-3}$ innerhalb der Meßgenauigkeit konstant.

\footnotetext{
${ }^{19}$ Der Kontrastverlust ist derzeit Gegenstand der Untersuchungen in der Specle-Interferometrie s. Langbein et al. (PRL 82, 1040 (1999))

${ }^{20}$ Die Methoden sind unterschiedlich sensitiv auf winkelabhängige Streuprozesse wie Kleinwinkelstreuung.
} 
Fehlerquellen bei dieser Methode liegen insbesondere in der Bestimmung der Probendicke $\mathrm{d}=3.8 \pm 0.2 \mu \mathrm{m}$, die mit interferometrischen Methoden ermittelt wurde, und den Mehrfachreflexionen, die durch die Glastasche entstehen, in welcher sich die Probe befindet.

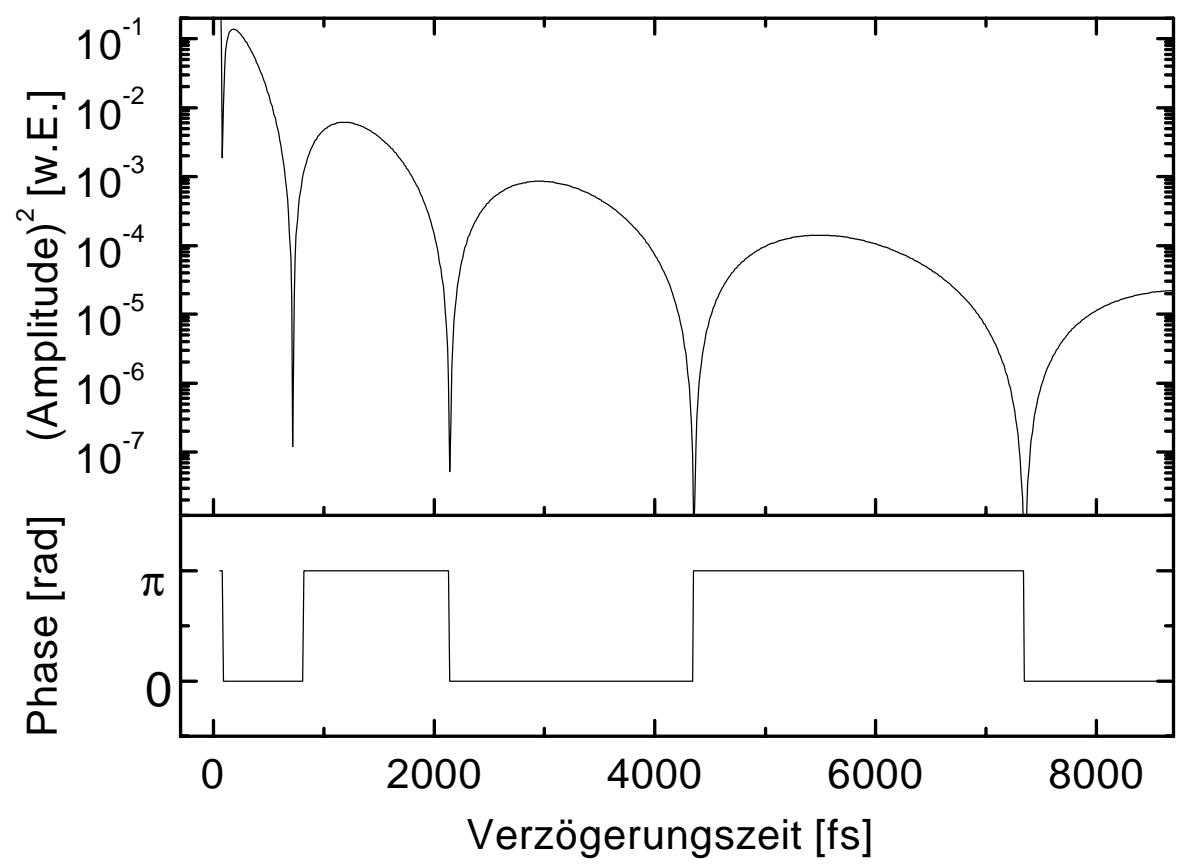

Abb. 4.2: Simulierte Amplituden und Phasenverläufe nach Panzarini (Gl. 2.25)

\subsubsection{Polariton-Pulspropagation bei niedrigen Anregungsdichten}

Bei konstant gehaltenem Anregungsspektrum wurden für niedrigste Anregungsfluenzen von $1.2 \mathrm{~mW} / \mathrm{cm}^{2}$ bis Fluenzen von $0.4 \mathrm{~W} / \mathrm{cm}^{2}$ gleiche Amplituden- und Phasenverläufe (s. Abb. 4.3 oben) gemessen. Für diese Anregungen befindet sich das System im linearen Regime. Der Zerfall der Amplitude wird von der Streuung des 1s-Exzitons an Störstellen bzw. Phononen beherrscht. Die Dämpfungskonstante des $1 \mathrm{~s}$-Exzitons beträgt rund $\hbar \gamma=30 \mu \mathrm{eV}$, welches ungefähr der Streurate der Exzitonen an piezoelektrisch aktiven TA- und LA- Phononenmoden entspricht [70]. Zusätzlich $\mathrm{zu}$ den 1s-Exziton Propagationsschwebungen erkennt man eine zeitlich langsame Modulation in der Amplitude bei 3 bzw. 6 ps, die auch in der Phase wiederzufinden ist. Durch Simulation des transmittierten elektrischen Feldes mit der dielektrischen Funktion nach Tanguy (Gl. 2.13) kann sie der 2s-Exzitonresonanz zugeordnet werden (s. Abb. 4.3 unten). Man findet eine stärkere Verbreiterung für den 2s- als für 

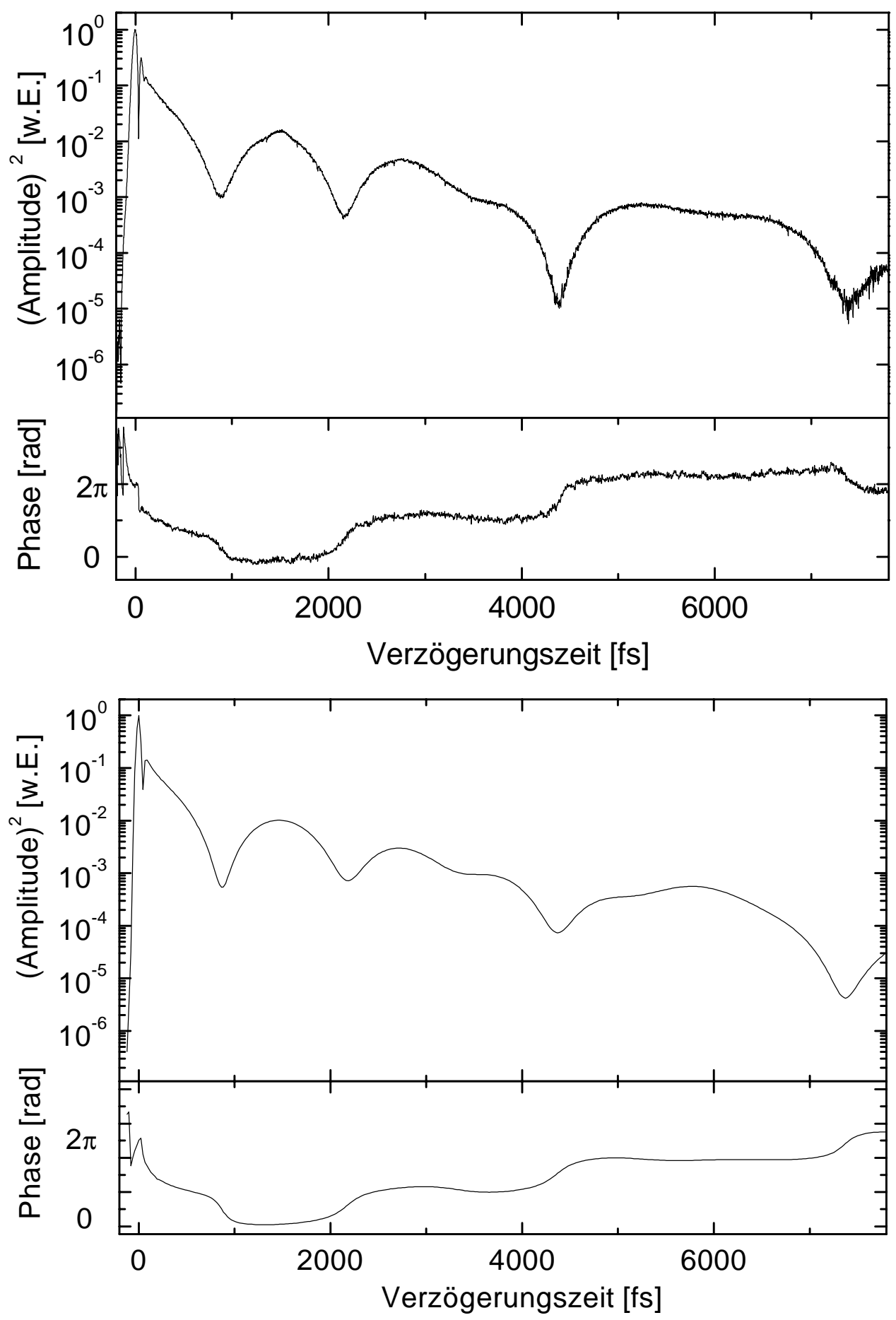

Abb. 4.3: Verläufe der Amplitudenquadrate und Phasen. Oben: Experiment. Unten: Mit Tanguys Formel simulierte Kurven. (Parameter s. Text) 
den 1s-Exzitonzustand: $\Gamma_{2 \mathrm{~s}} \cong 5 \cdot \Gamma_{1 \mathrm{~s}}$. Heuristisch läßt sich der Unterschied durch die größere räumliche Ausdehnung des 2s-Zustandes (Gl. 2.9) und seinem damit verbundenen größeren Streuquerschnitt erklären.

Im Niederdichtelimes wird der Nachweis von $1 s$ - $2 s$-Exzitonschwebungen in einem 3d-Kristall experimentell realisiert und gute Übereinstimmung zwischen Experiment und Theorie im Rahmen einer Beschreibung mit einer Lorentz verbreiterten dielektrischen Funktion für den Halbleiter gefunden.

Es besteht eine Diskrepanz zwischen Experiment und Theorie, was die Richtung des Phasenumklappens für $\tau=7.4 \mathrm{ps}$ und den Amplitudenverlauf bei 6 ps betrifft. Hierzu ist anzumerken, daß selbst im Niederdichtelimes das lineare Absorptionsspektrum aus theoretischer Sicht noch nicht vollständig verstanden ist und Diskrepanzen bezüglich Linienform und Lage der Resonanzlinien zwischen Theorie und Experiment bestehen. Siehe hierzu auch die asymmetrische 1s-Exziton Absorptionslinie (Abb. 3.2). Als Ursache für diese Unterschiede kommen räumliche Dispersion [25], die Entartung der Valenzbänder [71], Gitterschwingungen [72] oder Unordnung [73] in Betracht. 


\subsubsection{Polariton-Pulspropagation bei mittleren Anregungsdichten}

Die Amplitudenverläufe für variierende Fluenzen im Bereich von 0.4 bis 423 $\mathrm{mW} / \mathrm{cm}^{2}$ sind in Abb. 4.4 zusammengefaßt. Es wird eine starke Abhängigkeit des Abklingverhaltens der Amplitude von der Laserfluenz beobachtet. Der Einfluß des 2 s-Exzitons ist nur bei Fluenzen $<0.4 \mathrm{~W} / \mathrm{cm}^{-2}$ sichtbar und wird bei höheren Fluenzen schnell ausgedämpft.

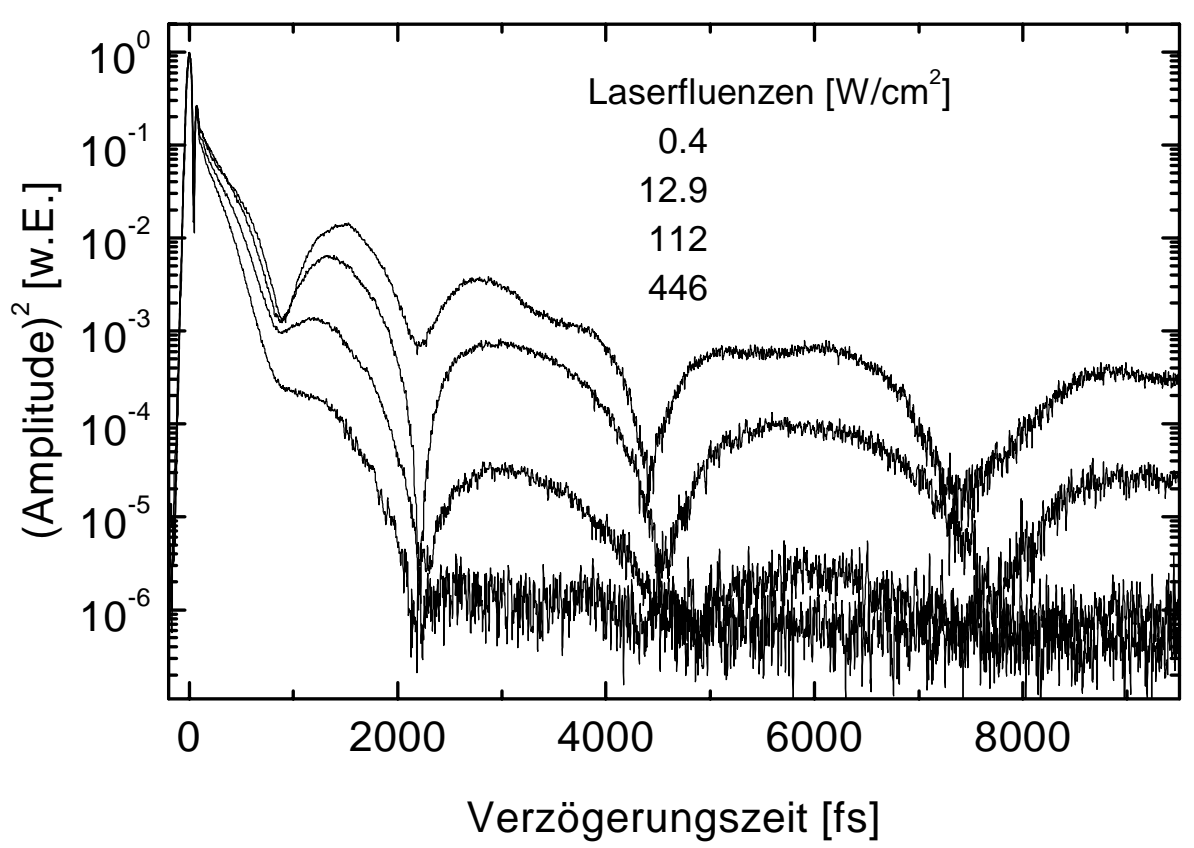

Abb. 4.4: Gemessene Amplitudenquadratverläufe für verschiedene Laserfluenzen. 


\subsubsection{Bestimmung des relevanten Streukanales}

Um das Abklingverhalten der gemessenen Amplitudenverläufe der PolaritonPulspropagation in Abhängigkeit der Anregungsfluenz $\mathrm{zu}$ verstehen, muß der dominierende Streukanal bestimmt werden. Die Streuung an thermischen Phononen trägt zu einem Streuhintergrund bei, dessen Änderung jedoch bei den betrachteten Anregungsdichten vernachlässigt werden kann, da sich die Probe im suprafluiden Helium befindet und so eine Temperatur der Probe unterhalb von $4.2 \mathrm{~K}$ gewährleistet wird $^{21}$. Der Einfluß von nicht-thermischen Phononen spielt im Vorgriff auf Kap. 4.2.2 eine untergeordnete Rolle. Durch die spektrale Breite der Anregung werden

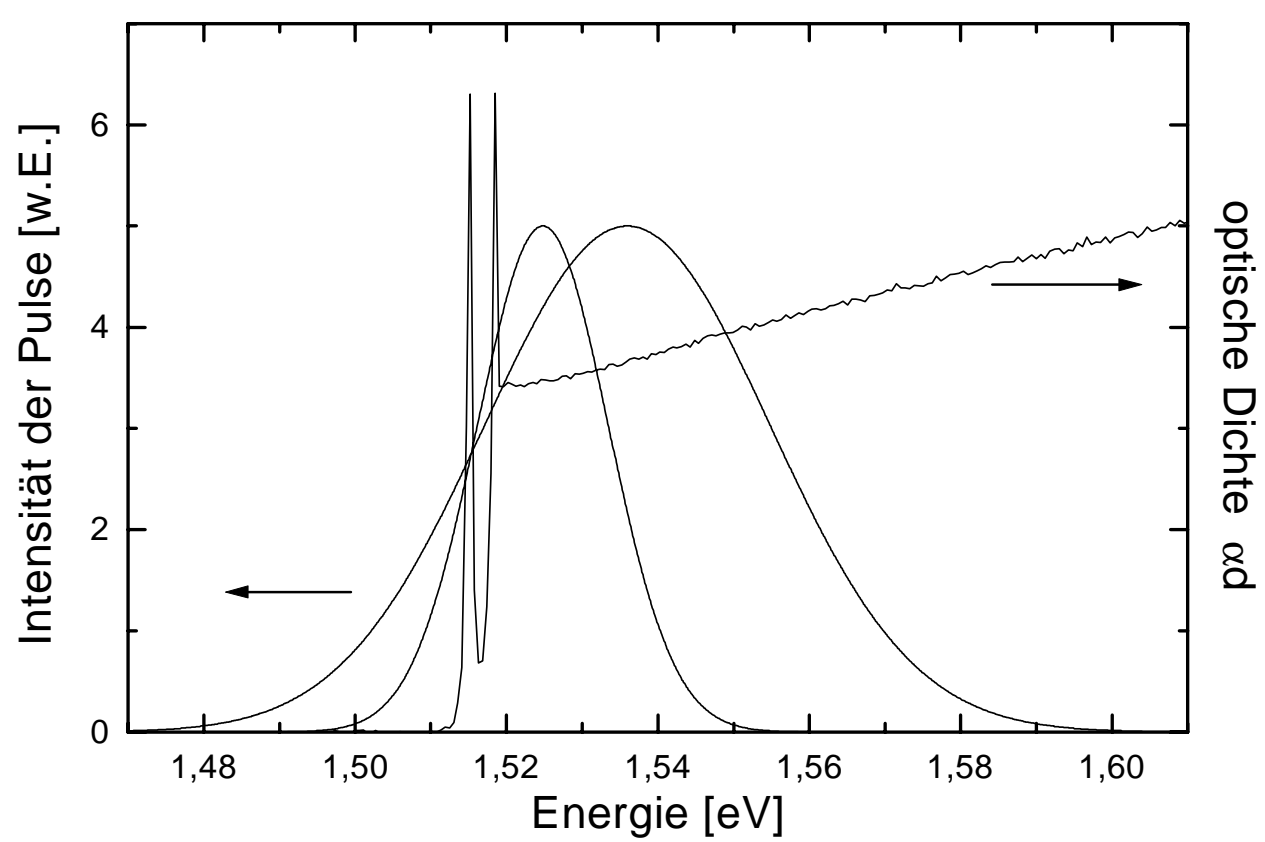

Abb. 4.5: Variation der spektralen Breite des Anregungsspektrum zur unterschiedlichen Gewichtung von freien und gebundenen Ladungsträgern. Zum Vergleich ein Absorptionsspektrum von GaAs bei $2 \mathrm{~K}$.

sowohl gebundene Exzitonen als auch Elektron-Loch-Kontinuumspaare generiert. Als Streumechanismen kommen daher sowohl Exziton-Exziton-Streuung als auch Ladungsträger-Exziton-Streuung in Frage. Durch Variation des Spektrums der Anregungspulse wird das relative Verhältnis der Besetzungszahlen des 1sExzitonzustandes bzw. der Kontinuumszustände variiert: Ein spektral schmalerer Puls generiert bei konstant gehaltener Dichte an 1s-Exztionen weniger freie e-hPaare im Kontinuum als ein spektral breiterer Puls (s. Abb. 4.5).

\footnotetext{
${ }^{21}$ Für Laserintensitäten größer als $1 \mathrm{~mW}$ wird der Siedepunkt von Helium (4.2 K) überschritten, welches man deutlich auf der CCD erkennt, auf die die Probe abgebildet wird.
} 
Die Anregungsdichte im Kontinuum wird aus dem spektralen Überlapp der Laserpulse mit dem optischen Dichtespektrum ermittelt. Mit der Fluenz $\mathrm{F}_{\text {vor }}$ des Laserstrahls vor der Probe, der Repetitionsrate der Pulse $f_{\text {rep }}=80 \mathrm{MHz}$, der Dicke d der Probe, dem Reflexionskoeffizienten R und der mittleren Energie E der Photonen bestimmt sich die Anregungsdichte im Kontinuum $\mathrm{n}_{\text {kont }}$ mit

$$
\mathrm{n}_{\text {kont }}=(1-\mathrm{R}) \cdot \frac{\mathrm{F}_{\mathrm{vor}} \cdot(1-\exp (-\alpha \mathrm{d}) \cdot \mathrm{C})}{\mathrm{E} \cdot \mathrm{d} \cdot \mathrm{f}_{\text {rep }}}
$$

Die Konstante $\mathrm{C}$ berücksichtigt den spektralen Überlapp des Absorptionsspektrums der Probe mit dem Anregungsspektrum. Die Fluenz $F_{\text {vor }}$ unmittelbar vor der Probe berechnet sich aus der gemessenen Leistung $\mathrm{P}_{\mathrm{vor}}$, dem Strahlparameter vor der Linse (Brennweite $f$ ) und der Wellenlänge $\lambda$ des verwendeten Lichtes

$$
\mathrm{F}_{\mathrm{vor}}=\mathrm{P}_{\mathrm{vor}} \cdot\left(\frac{2 \pi \cdot \mathrm{a}}{\lambda \mathrm{f}}\right)^{2} / \pi
$$

Das Verhältnis der Dichte von Elektron-Loch-Kontinuumspaaren zur Dichte von 1sExzitonen wurde durch spektrale Filterung der Anregungspulse mit der Dispersionsstrecke variiert (s. Abb. 3.5). Messungen mit spektral verschiedenen Pulsen [74] zeigen, daß der Einfluß der Exziton-Exziton-Streuung vernachlässigt werden kann.

Für Anregungen mit ultrakurzen Pulsen dominiert die Anregungsdichte im Kontinuum die Dephasierungszeit des 1 s-Exziton-Polaritons.

Dieses Ergebnis ist zu erwarten, da im Falle von ultrakurzen Pulsen der Überlapp des Pulsspektrums mit dem Kontinuum viel größer ist als der mit der 1s-Resonanz. Darüber hinaus ist aus zeitaufgelösten, entarteten Vier-Wellen-Mischexperimenten (DFWM) bekannt, daß die Streueffizienz der Kontinuum-Exziton-Streuung rund 10fach stärker ist als die der Exziton-Exziton-Streuung [75].

Bei der Bestimmung der Anregungsdichte wurde ein proportionaler Zusammenhang zwischen Laserfluenz und Dichte angenommen. Bei dieser Annahme ist jedoch Vorsicht geboten, da Streukanäle wie Oberflächenladungsträgerrekombination die tatsächlichen Dichten modifizieren können. 


\subsubsection{Abhängigkeit der Dämpfung von der Anregungsdichte}

Aus dem Amplitudenverläufen soll die Dämpfung der 1s-Exzitonen PolaritonPulspropagation in Abhängigkeit der Anregungsdichte bestimmt werden. Durch Vergleich der Meßdaten mit Simulationen kann jeder Kurve eine Dämpfungskonstante $\gamma_{1 \mathrm{~s}}$ zugeordnet werden. Bei dieser Bestimmung ist es entscheidend, welches Modell für die Simulationen zu Grunde gelegt wird. Es sollen drei Modelle betrachtet werden:

\section{Modell 1: Homogene Probe.}

Es wird angenommen, daß die optisch generierten Ladungsträger sich gleichmäßig über die ganze Probe verteilen, welche als räumlich homogen vorausgesetzt wird. Ausgangspunkt ist die dielektrische Funktion des direkten Halbleiters (Gl. 2.13). Das transmittierte elektrische Feld durch eine planparallele Platte wird mit Gl. 3.24 berechnet.

\section{Modell 2: Berücksichtigung des räumlichen Profils der Elektron-Lochpaare.}

Wie in Kap. 4.1.3.1. gezeigt wurde, hängt die Dephasierung des 1s-ExzitonPolaritons von der Anregungsdichte der e-h-Kontinuumspaare ab. Diese sind auf-

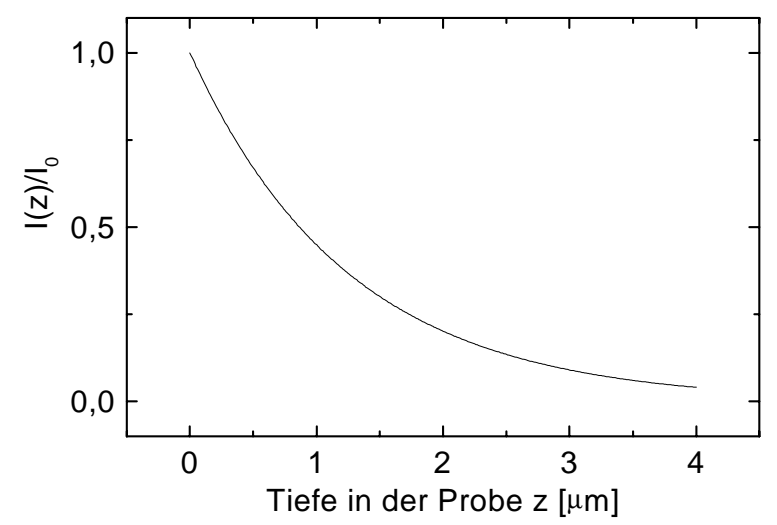

Abb. 4.6: Intensitätsverlauf, der durch den Absorptionskoeffizienten $\alpha=0.8 \mu \mathrm{m}^{-1}$ aufgeprägt wird.

grund des Absorptionskoeffizienten kurz nach der Anregung inhomogen in der Probe verteilt (s. Abb. 4.6). Aus Reflexionsmessungen mit Anregung an der Probenvorderund Hinterseite [76] geht hervor, daß die räumliche Ladungsträgerdiffusion auf der ps-Zeitskala vernachlässigt werden kann und somit das durch das Absorptionsprofil aufgeprägte Ladungsträgerprofil auf der im Experiment betrachteten Zeitskala 
erhalten bleibt. Mit dem Absorptionskoeffizienten im Kontinuum $\alpha$ wird für die Dämpfung des 1s-Exziton-Polaritons angesetzt

$$
\gamma_{1 \mathrm{~s}}\left(\gamma_{1}, \mathrm{z}\right)=\gamma_{0}+\gamma_{1}(\mathrm{n}) \cdot \mathrm{e}^{-\alpha \cdot \mathrm{z}}
$$

wobei $\gamma_{0}$ ein räumlich konstanter und dichteunabhängiger Streuhintergrund ist und $\gamma_{1}(\mathrm{n})$ den dichteabhängigen Beitrag darstellt, der bestimmt werden soll. Die Probe wird in Scheibchen unterteilt, in denen jeweils der komplexe Brechungsindex verwendet wird, der mit der dielektrischen Funktion des Einoszillatormodells Gl. 2.4 ohne räumliche Dispersion $(\omega(\mathrm{q})=$ const $)$ unter Verwendung von Gl. 4.3 bestimmt wird. Das transmittierte elektrische Feld wird näherungsweise mit

$$
E_{T}(\omega)=E_{0}(\omega) \cdot \frac{2}{\left.\tilde{n}(\omega)\right|_{z=0}+1} \cdot \frac{\left.2 \tilde{n}(\omega)\right|_{z=d}}{\left.\tilde{n}(\omega)\right|_{z=d}+1} \cdot e^{i \cdot \int_{0}^{d} d z \cdot \tilde{n}(\omega, z) \cdot \omega / c}
$$

berechnet (vgl. mit Gl. 3.26). Bei dieser Näherung (adiabatische Näherung) werden Mehrfachreflexionen zwischen den Schichten vernachlässigt. Hierfür spricht, daß Oberflächeneffekte (Transmissionkoeffizienten durch die Grenzflächen wie auch Mehrfachreflexionen) wenig Einfluß auf die Polariton-Pulspropagation in Transmission bei optisch dicken Proben für spätere Zeiten haben (s.a. [68]).

\section{Modell 3: Berücksichtigung des räumlichen Profils der Elektron-Lochpaare und der räumlichen Dispersion des 1s-Exzitons.}

Als weitere Verfeinerung des Modells 2 soll räumliche Dispersion des 1s-ExzitonPolaritons berücksichtigt werden. Mit der dielektrischen Funktion Gl. 2.4 wird unter Verwendung von Gl. 2.8 das transmittierte elektrische Feld $\mathrm{E}_{\mathrm{T}}(\omega)$ näherungsweise berechnet mit

$$
\begin{aligned}
\mathrm{E}_{\mathrm{T}}(\omega)=\mathrm{E}_{0}(\omega) \cdot & \left(\lambda _ { 1 } ( \omega ) \cdot \operatorname { e x p } \left(\mathrm{i} \cdot \int_{0}^{\mathrm{d}} \mathrm{dx} \cdot \mathrm{q}_{1}(\omega, \mathrm{x})+\right.\right. \\
+ & \lambda_{2}(\omega) \cdot \exp \left(\mathrm{i} \cdot \int_{0}^{\mathrm{d}} \mathrm{dx} \cdot \mathrm{q}_{2}(\omega, \mathrm{x})\right)
\end{aligned}
$$

wobei die beiden Faktoren

$$
\lambda_{1}(\omega)=\frac{\chi\left(\omega, \mathrm{q}_{1}(\omega)\right)}{\chi\left(\omega, \mathrm{q}_{2}(\omega)\right)-\chi\left(\omega, \mathrm{q}_{1}(\omega)\right)} \text { und } \lambda_{2}(\omega)=\frac{\chi\left(\omega, \mathrm{q}_{2}(\omega)\right)}{\chi\left(\omega, \mathrm{q}_{1}(\omega)\right)-\chi\left(\omega, \mathrm{q}_{2}(\omega)\right)}
$$


durch Pekars Randbedingung (Gl. 2.20) an der Vorderseite der Probe festgelegt werden und die beiden Polaritonmoden $\mathrm{q}_{1,2}(\omega, \mathrm{x})$ durch die Lösung der Polaritongleichung (Gl. 2.14) gegeben sind. Hierbei werden sowohl Oberflächeneffekte als auch Streuprozesse von der einen Polaritonmode in die andere zur selben Energie vernachlässigt. Für eine korrekte Beschreibung der Streuwahrscheinlichkeiten von der einen Mode in die andere müßten zusätzliche Randbedingungen an jedem Übergang von Scheibe zu Scheibe angewendet werden, was aber wegen des heuristischen Modells der Pekarschen Randbedingungen problematisch ist und deshalb hier nicht weiter verfolgt werden soll. Für verschwindendes räumliches Profil $(\alpha \rightarrow 0)$ entspricht dieses Modell zur räumlichen Dispersion dem von Schäfer [68] angewandten Modell zur Beschreibung einer Anomalie im Transmissionsspektrum von GaAs.

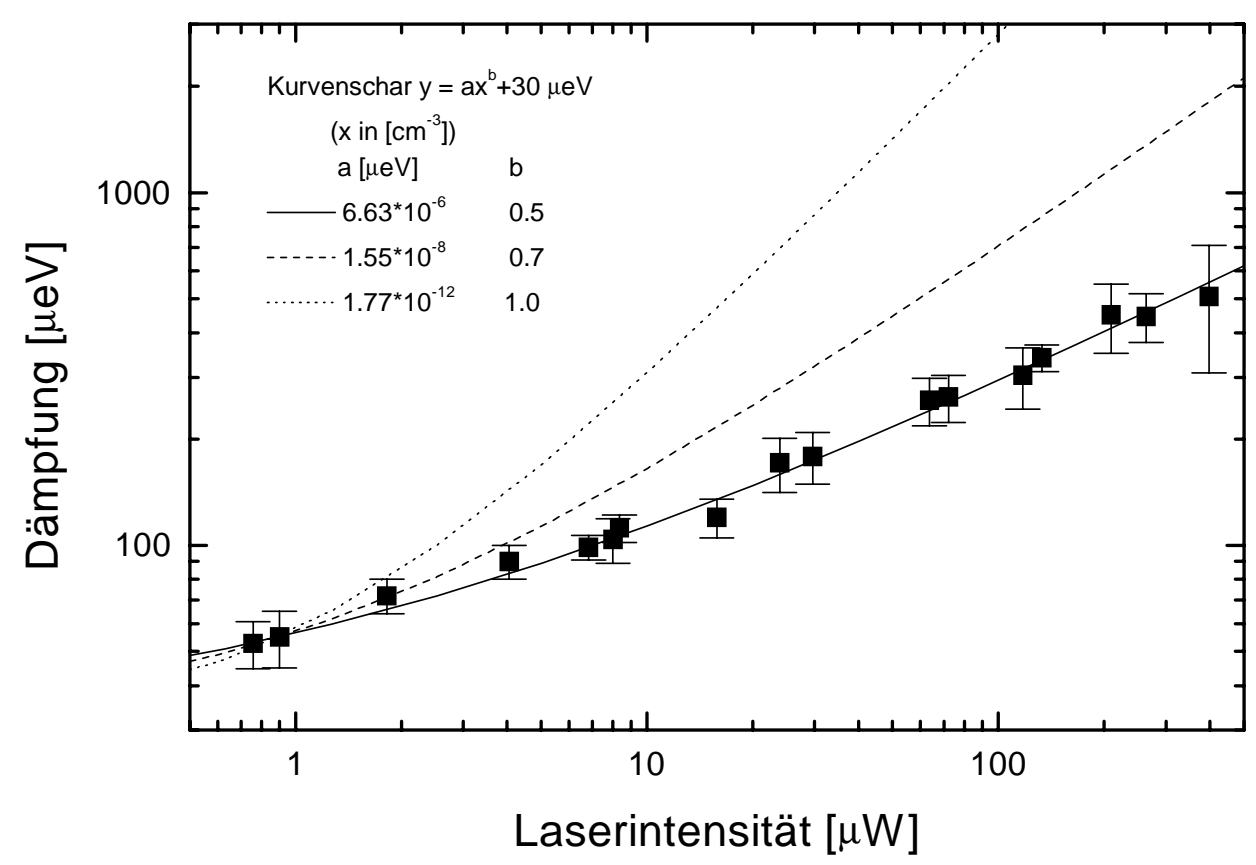

Abb. 4.7: Effektive Dämpfung, die unter der Annahme bestimmt wurde, daß sich die optisch generierten Ladungsträger homogen über die Probe verteilen (Modell 1).

Es werden die typischen Stoffkonstanten von GaAs verwendet (Masse des Exzitons: $\left.0.5 \cdot \mathrm{m}_{0}, \mathrm{f}=86 \mu \mathrm{eV}, \hbar \omega_{0}=1.515 \mathrm{eV}, \alpha_{\hbar \omega=1.52 \mathrm{eV}}=0.8 \mu \mathrm{m}\right)$. Als Probenparameter gehen die Hintergrundsdämpfung $\left(\gamma_{0}=30 \mu \mathrm{eV}\right)$ und die Probendicke ein. Abb. 4.7 zeigt den Verlauf der effektiven Dämpfung, die mit dem Modell 1 bestimmt wird. Die mittlere Anregungsdichte wurde mit Gl. 4.1 aus der Laserintensität berechnet. Abb. 4.8 zeigt den Verlauf des dichteabhängigen Anteils der Dämpfung $\gamma_{1}$ als Funktion der Laserintensität im Modell 2. 
Bei aller Schwierigkeit, den Exponent aus den Kurven zu bestimmen, ist doch ein sublinearer Verlauf der Dämpfung in Abhängigkeit von der Anregungsdichte für die Modelle 1 und 2 festzustellen. Die Sublinearität ist bei Modell 1 stark ausgeprägt, wo sie eine fast wurzelförmige Abhängigkeit zeigt.

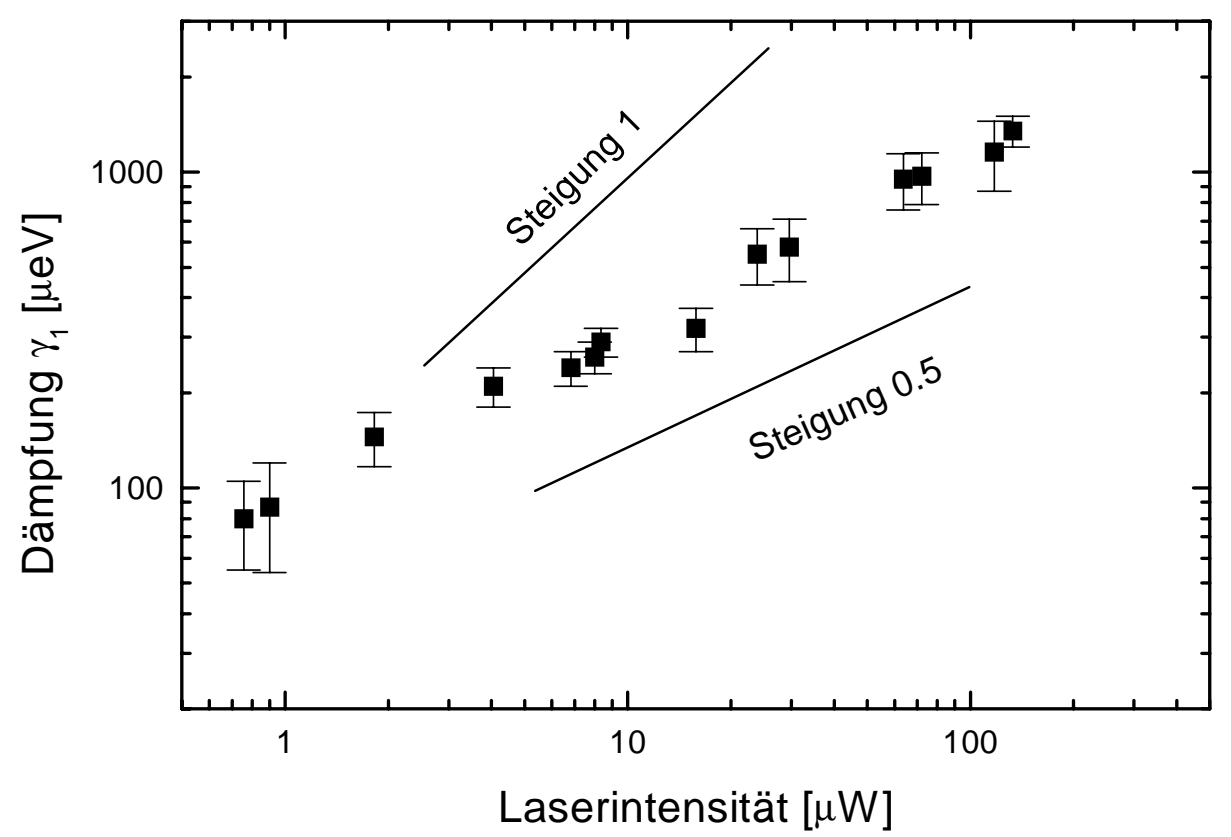

Abb. 4.8: Dichteabhängiger Anteil der Dämpfung, der unter der Annahme einer Ladungsträgerverteilung, die dem Absorptionsprofil folgt, bestimmt wurde (Modell 2).

Die Dämpfung des 1s-Exziton-Polaritons steigt im Rahmen der Beschreibung der Modelle 1 und 2 sublinear mit der Anregungsdichte von Elektron-LochKontinuumspaaren an!

Ein sublinearer Zusammenhang steht im Widerspruch zu einfachen Streutheorien in erster Bornscher Näherung, die einen linearen Zusammenhang zwischen Dämpfung und Dichte der Streuzentren $n$ liefern

$$
\gamma(\mathrm{n})=\gamma_{0}+\alpha \cdot \mathrm{n}
$$

Hierbei steht $\gamma_{0}$ für einen konstanten Steuhintergrund und $\alpha$ für die Streueffizienz.

In der Literatur wird erst in jüngster Zeit auf eine experimentelle Beobachtung eines sublinearen Zusammenhanges eingegangen, wie z.B. in den Vierwellenmischexperimenten von Allan et al. [77] an InP bei denen jedoch keine weitere Erklärung 
gegeben wird. Zeitaufgelöste Transmissionsmessungen an $\mathrm{ZnS}_{\mathrm{x}} \mathrm{Se}_{1-\mathrm{x}} / \mathrm{ZnSe}$ Heterostrukturen zeigen Sättigungseffekte, werden aber ebenfalls nicht weiter diskutiert [78]. Das Ergebnis steht im Widerspruch zu den Ergebnissen aus Vierwellenmischexperimenten von Schultheis/Kuhl an GaAs [79,80], in denen eine Linienbreitenanalyse auf Basis der optischen Blochgleichungen durchgeführt wurde. Diese Experimente wurden allerdings an sehr viel dünneren Proben (100 -190 nm) durchgeführt, so daß mögliche Propagationseffekte, die einen sublinearen Zusammenhang liefern könnten, weniger ins Gewicht fallen. Ein weiterer Grund für eine Diskrepanz könnten auch die bei zeitintegrierten Vierwellenmischexperimenten auftretenden Schwierigkeiten bei der Unterscheidung des freien Polarisationszerfalls und des Photonenechos sein, welches zusammen zu nichtexponentiellen Abklingkurven führt [81]. Ein möglicher Grund für einen sublinearen Zusammenhang könnte die Oberflächenladungsträgerrekombination sein, die bei einer Probe ohne Schutzschicht, wie sie bei der vorliegenden Arbeit verwendet wurde, insbesondere bei höheren Anregungsdichten zu einer Überschätzung der tatsächlichen Ladungsträger führt.

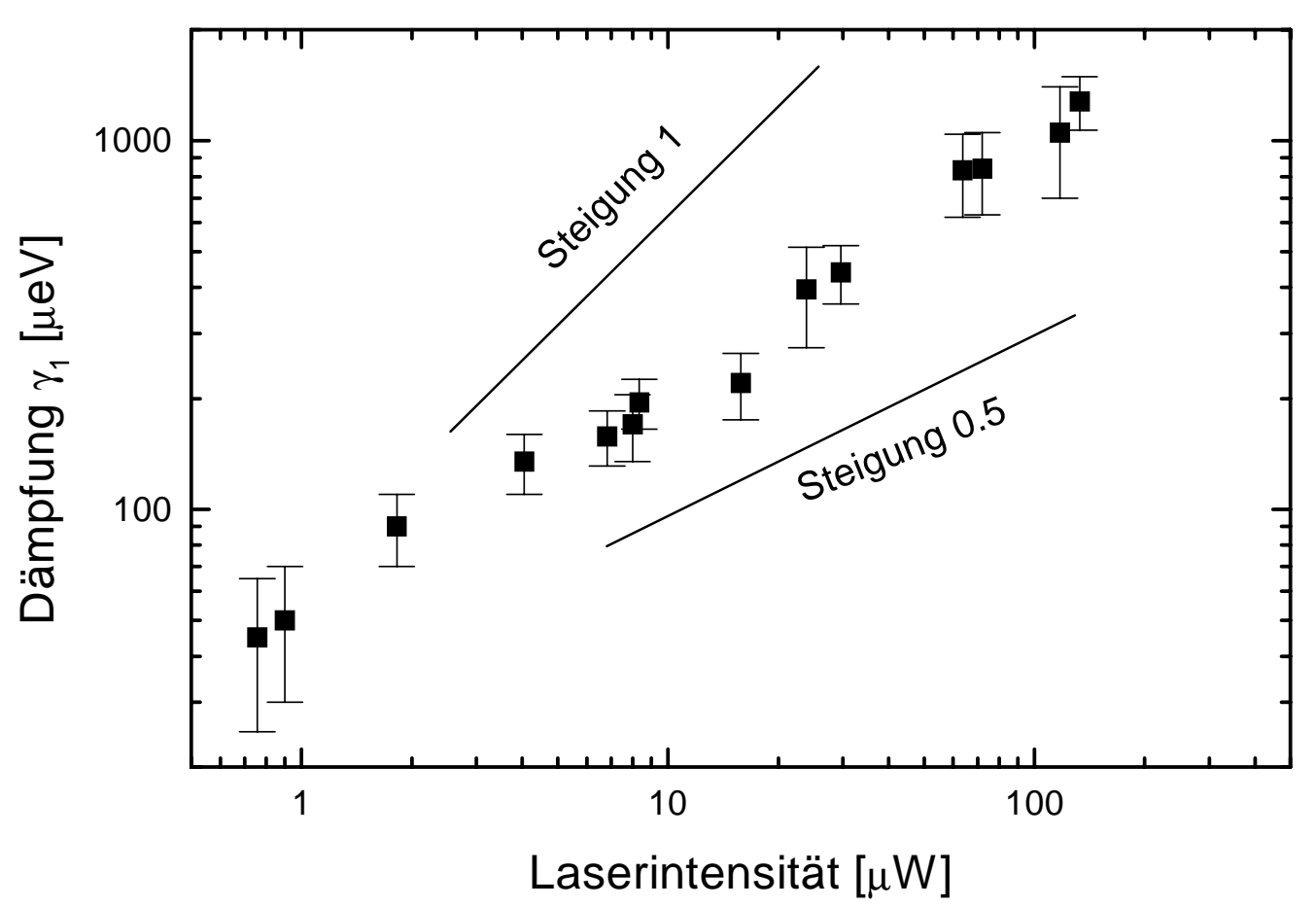

Abb. 4.9: Dichteabhängiger Anteil der Dämpfung, der unter Annahme einer Ladungsträgerverteilung, die dem Absorptionsprofil folgt, und mit räumlicher Dispersion für das 1s-Exziton bestimmt wurde (Modell 3). 
Abb. 4.9 zeigt den dichteabhängigen Anteil der Dämpfung im Modell 3. Die Steigung ist im Vergleich zu Modell 2 etwas größer. Die Frage, ob räumliche Dispersion in Verbindung mit einem räumlichen Profil der Ladungsträger in der Lage ist, die Meßdaten unter Annahme eines linearen Zusammenhanges zwischen Dämpfung und Anregungsdichte zu erklären, bedarf jedoch weitergehenden Untersuchungen. Bemerkenswert ist, daß räumliche Dispersion als Propagationseffekt mit dem räumlichen Profil, den funktionalen Zusammenhang zwischen Dämpfung und Anregungsdichte modifiziert. Von einem derartigen Effekt wurde meines Wissens nach noch nicht in der Literatur berichtet.

Es ist zu bemerken, daß die Minima der mit Modell 2 und 3 berechneten Amplitudenkurven für große Dämpfungen zu späteren Zeiten schieben. Ursache hierfür ist, $\mathrm{da}$ an der Probenvorderseite die optischen Eigenschaften durch die starke Dämpfung strukturlos werden, so daß die Probe optisch dünner erscheint. Dieser Effekt ist im Modell 3 weniger ausgeprägt. Ferner ist an den einzelnen Amplituden- bzw. Phasenkurven nicht zu erkennen, ob eine Kurve mit oder ohne räumlicher Dispersion bzw. mit oder ohne räumlichem Profil berechnet wurde, wenn man eine Ungenauigkeit in der Amplitude zuläßt, die dem experimentellen Fehler entspricht. Erst das Verhalten der Dämpfung in Abhängigkeit von der Anregungsdichte zeigt Unterschiede. Legt man Pekars Randbedingung zu Grunde, ist der Einfluß der räumlichen Dispersion auf die Phasenverläufe zu vernachlässigen.

In der vorangegangenen Diskussion wurde stets das Signal für spätere Zeiten betrachtet. Das Signal für frühere Zeiten $(<1 \mathrm{ps})$ und höhere Anregungsdichten wird in Simulationen (etwa mit der Tanguyformel (Gl. 2.13) mit oder ohne Berücksichtigung des räumlichen Profils der Ladungsträger (Gl. 4.3)) nur ungenau wiedergegeben. Das Verschwinden der Ausbeulung für steigende Laserfluenzen bei 600 fs wird nicht reproduziert (vgl. Abb. 4.10 und Abb. 4.4). Interessanterweise scheint das Modell ohne räumlichem Profil der Ladungsträger den charakteristischen Verlauf der Meßdaten für frühere Zeiten besser wiederzugeben.

Eine Diskrepanz zwischen Experiment und dem heuristischen Modell nach Tanguy ist zu erwarten, da die optischen Eigenschaften des Halbleiters nahe der Bandkante durch die Veränderung der Rydberg- sowie der Bandlückenenergie bzw. der energieund dichteabhängigen Dephasierung durch die Anwesenheit der Ladungsträger modifiziert werden. Eine genaue Beschreibung des Signals für frühe Zeiten erfordert die volle Beschreibung des Halbleiters im Rahmen der Halbleiter-Blochgleichungen. 


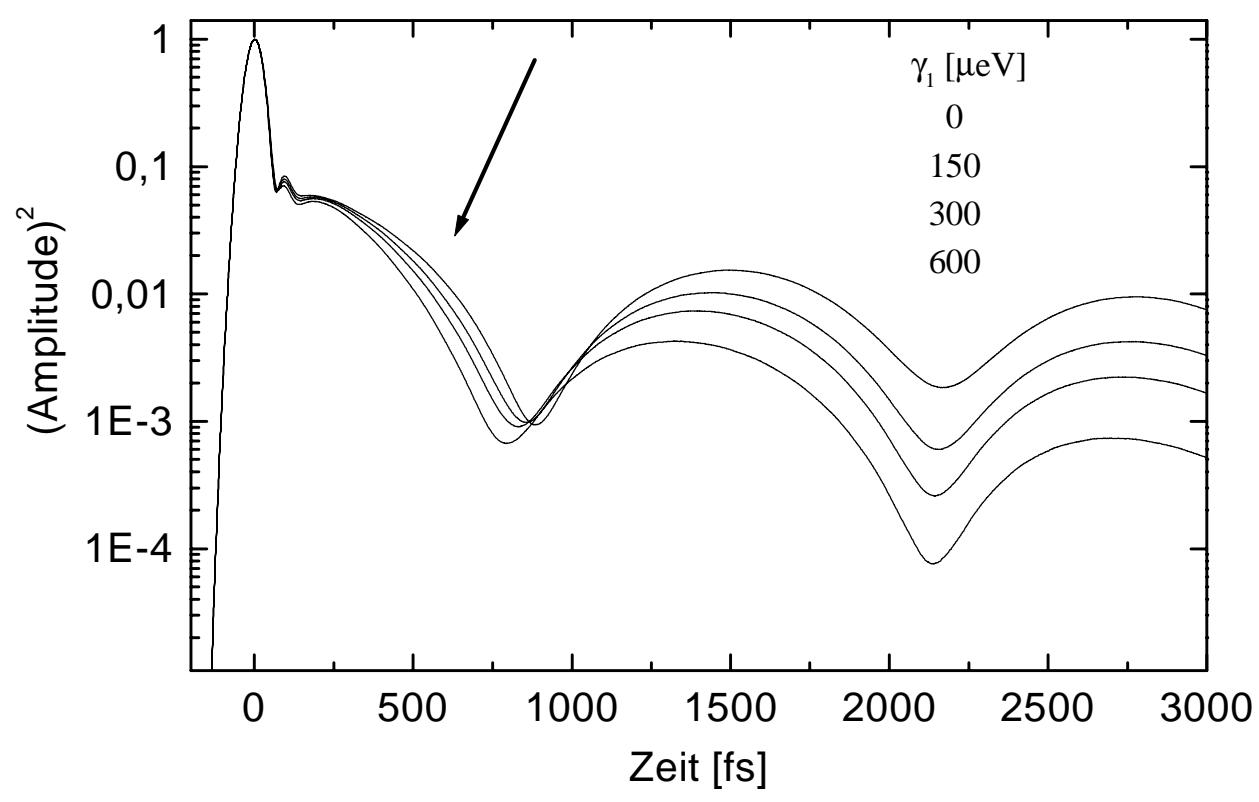

Abb. 4.10: Mit Tanguys Formel berechnete Amplitudenverläufe unter Verwendung von Gln. 4.3 und 4.4 für verschiedene $\gamma_{1}$ mit $\gamma_{0}=30 \mu \mathrm{eV}$. Die Schulter (Pfeil) verschwindet nicht für höhere Dämpfungen wie es im Experiment beobachtet wird (s. Abb. 4.4). Das Signal für Zeiten $<300$ fs ist von der spektralen Lage des Laserpulses abhängig. 


\subsubsection{Abhängigkeit der Phase von der Anregungsdichte}

Die Oszillationen bei der Polariton-Pulspropagation werden durch die Schwebung der Polaritonwellenpakete auf den beiden Polaritonäste verursacht. Der Unterschied zu einer üblichen Schwebung (s. Kap 3.2) besteht darin, daß die Amplituden und Frequenzen der beiden Einzelschwinger zeitabhängig sind, da sie mit den Gruppengeschwindigkeiten und den Dämpfungen der beiden Polaritonäste verknüpft sind. Mit Hilfe der Phase kann somit Information über den zeitlichen Verlauf der Amplituden der am hinteren Ende der Probe ankommenden Polaritonwellenpakete gewonnen werden.

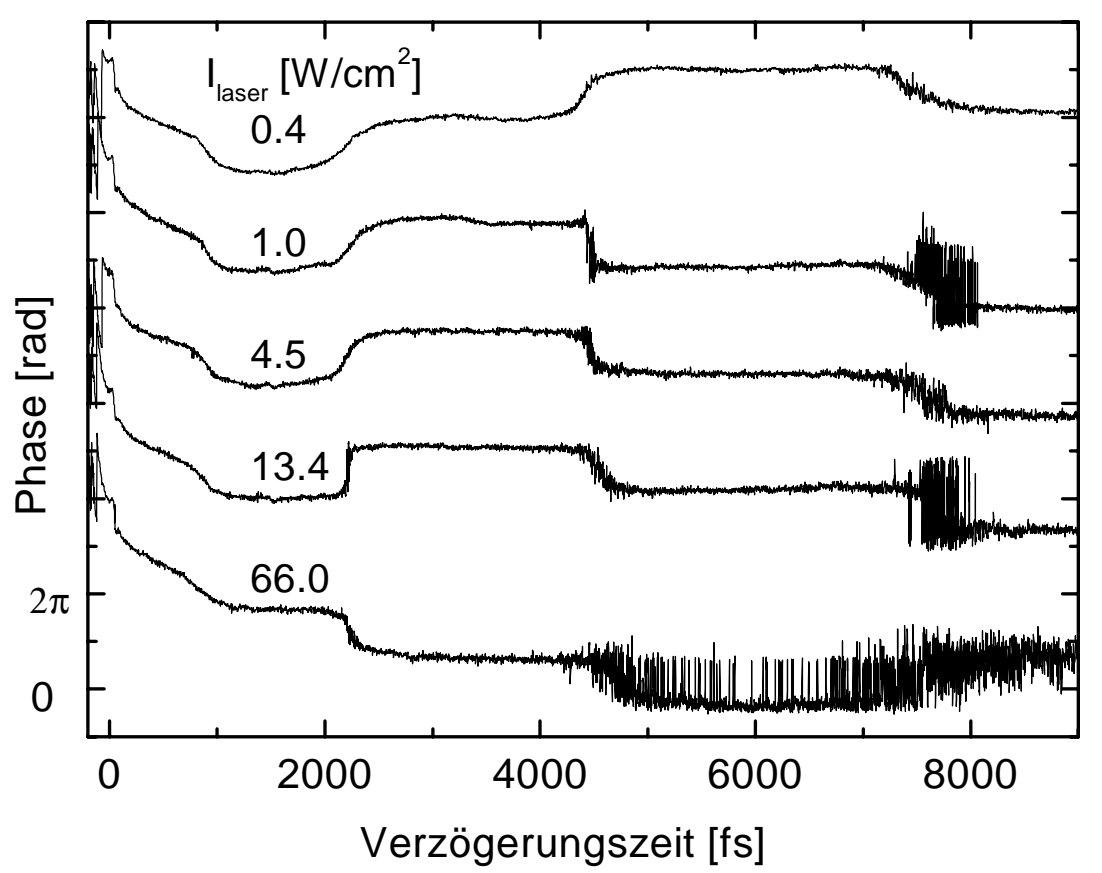

Abb. 4.11: Gemessene Phasenverläufe bei unterschiedlichen Laserfluenzen.

In den Anregungsdichte abhängigen Messungen (s. Abb. 4.11) ist zu erkennen, daß die Phasensprünge mit steigender Anregungsdichte nach unten klappen. Das mit der Anregungsdichte größer werdende Rauschen des Phasensignals für spätere Zeiten ist durch die verschwindende Intensität des Signals verursacht. Charakteristisch ist das anfängliche Aufsteilen der Phase bis zu einer Stufenform, ihr Richtungswechsel („Umklappen“) und ihr erneutes Weichwerden. Bei steigender Anregungsdichte klappen zuerst Phasensprünge für spätere Zeiten um, Phasensprünge für frühere 
Zeiten folgen. Die Resonanzfrequenz des 1s-Exzitons (= mittlere Trägerfrequenz, bezüglich der die Phase ausgewertet wird) bleibt für die betrachteten Anregungsintensitäten in Übereinstimmung mit Ergebnissen in der Literatur [82] konstant.

Dieser charakteristische Phasenverlauf läßt sich durch Resonanzlinien mit Lorentzform nicht erklären: Der symmetrische Imaginärteil und der asymmetrische Realteil der dielektrischen Funktion des harmonischen Oszillators (Gl. 2.4) führen zu stets aufsteigenden Phasensprüngen. Ebenso liefert die Tanguyformel (Gl. 2.13) nur aufsteigende Sprünge. Wird in der Tanguyformel die Dämpfung so groß, daß das 1sExziton mit dem Kontinuum verschmilzt und somit eine Asymmetrie induziert wird, klappt zuerst der erste, dann die Phasensprünge für spätere Zeiten um.

Mit lorentzverbreiterten Linien kann man den charakteristischen Phasenverlauf nicht erklären. Zur Interpretation ist eine andere Linienform notwendig.

Im einfachsten Fall kann eine Asymmetrie durch die Summe zweier Oszillatoren mit unterschiedlichen Dämpfungen, Oszillatorstärken und verschobenen Resonanzfrequenzen induziert werden, deren energetische Verschiebung vergleichbar gegenüber den Dämpfungen ist. Die dielektrische Funktion ergibt sich durch Superposition

$$
\varepsilon(\omega)=\varepsilon_{\mathrm{b}} \cdot\left(1+\frac{\mathrm{f}_{1}}{\omega_{0,1}^{2}-\omega^{2}-2 \cdot \mathrm{i} \gamma_{1} \cdot \omega}+\frac{\mathrm{f}_{2}}{\omega_{0,2}^{2}-\omega^{2}-2 \cdot \mathrm{i} \gamma_{2} \cdot \omega}\right)
$$

Da unter Verwendung von Pekars Randbedingung räumliche Dispersion keinen starken Einfluß auf die Phase hat, soll sie in dieser Betrachtung zur besseren Übersichtlichkeit vernachlässigt werden.

Für die Simulation der folgenden Phasenverläufe wurde eine $4 \mu \mathrm{m}$ dicke Probe angenommen und für die beiden Resonanzen folgende Werte verwendet: Die Oszillatorstärken sind gleichgewichtet $\mathrm{f}_{1}=\mathrm{f}_{2}=40 \mu \mathrm{eV}$, die Dämpfung der energetisch höherliegenden Resonanz ist doppelt so groß $\gamma_{2}=2 \cdot \gamma_{1}$ und die Resonanzfrequenzen sind um den energetischen Betrag von $\Delta \mathrm{E}$ gegeneinander verschoben $\hbar \omega_{0 \pm}=1.515 \mathrm{eV} \pm$ $\Delta \mathrm{E} / 2$

Die Superposition zweier Lorentzlinien zu einer asymmetrischen Resonanz ist in Abb. 4.12 zu sehen. Während in der Amplitude kein wesentlicher Unterschied zu erkennen ist, ob es sich um eine symmetrische oder asymmetrische Resonanz 
handelt, ist der zeitliche Verlauf der Phase sehr sensitiv davon abhängig (s. Abb. 4.13).

\begin{tabular}{|l|l|l|}
\hline Fall & $\begin{array}{l}\text { rel. Verschiebung } \\
\Delta \mathrm{E}[\mathrm{eV}]\end{array}$ & $\begin{array}{l}\text { Dämpfung } \\
\hbar \gamma_{1}[\mu \mathrm{eV}]\end{array}$ \\
\hline a) & 0 & 100 \\
\hline b) & 60 & 200 \\
\hline c) & 100 & 200 \\
\hline d) & 200 & 200 \\
\hline e) & 400 & 200 \\
\hline f) & 400 & 400 \\
\hline
\end{tabular}

Tab. 4.1: Verwendete Parameter zur Simulation der Phasenverläufe.

Die Phase klappt für eine zunehmende Asymmetrie für frühere Zeiten um. Für den Effekt spielt die energetische Lage des Schwerpunkts der Doppelresonanz keinerlei Rolle, sie bestimmt lediglich die mittlere Steigung der Phase. Wesentlich ist die Asymmetrie des Profils der Resonanz, so daß man folgern kann:

Die steigende Anregungsdichte induziert eine zunehmende Asymmetrie der 1s-Exzitonresonanz mit mehr Gewicht auf der höher energetischen Seite, die zu einem Umklappen der Phase führt!

Ein derartiger intensitätsabhängiger Phaseneffekt ist neu ([74, 83]). In der Literatur werden Veränderungen der 1s-Exziton-Linienform erst ab Ladungsträgerdichten oberhalb von $10^{15} \mathrm{~cm}^{-3}$ berichtet, der hier beobachtete Effekt findet bei extrem geringen Dichten ${ }^{22}$ von rund $6 \cdot 10^{13} \mathrm{~cm}^{-3}$ statt. Als Grund für die Asymmetrie kommt die frequenzabhängige Dämpfung (Kap. 2.3.2) in Frage.

${ }^{22}$ Die Dichte wurde mit Gl. 4.1 bestimmt. 


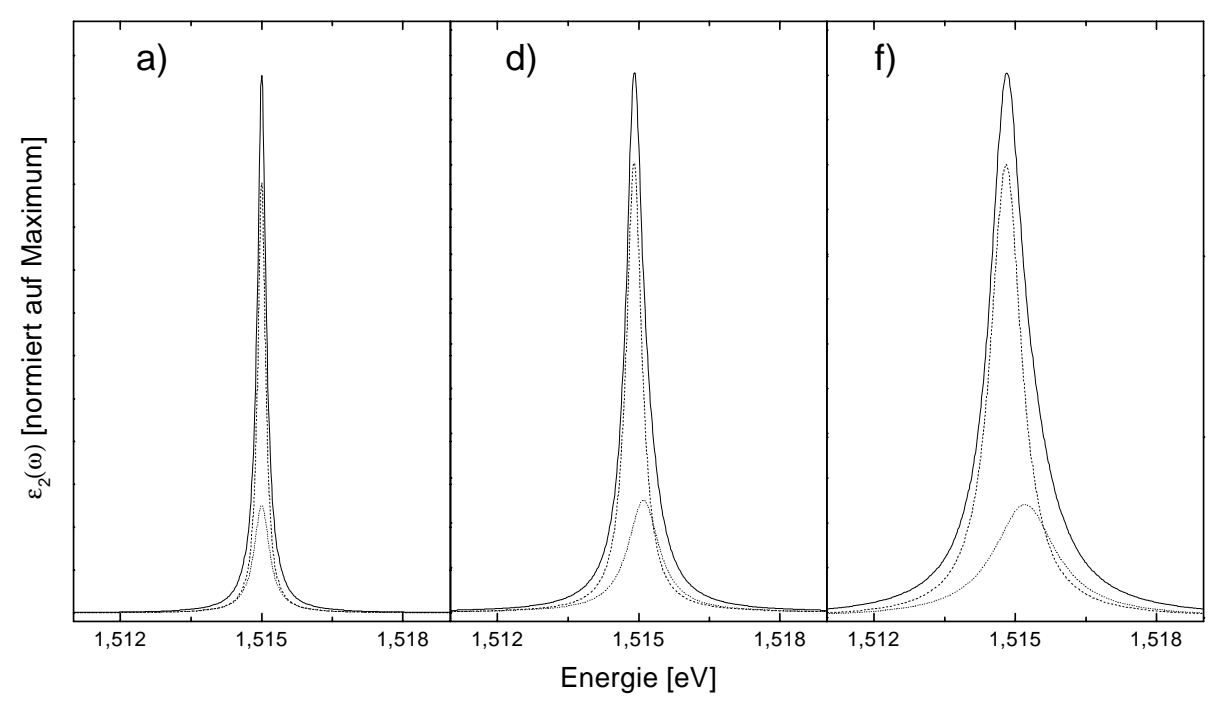

Abb. 4.12 : Imaginärteil der Superposition zweier Lorentzlinien zu einer asymmetrischen Resonanz für die Fälle a), d) und f). Gestrichelt: Einzellinien.

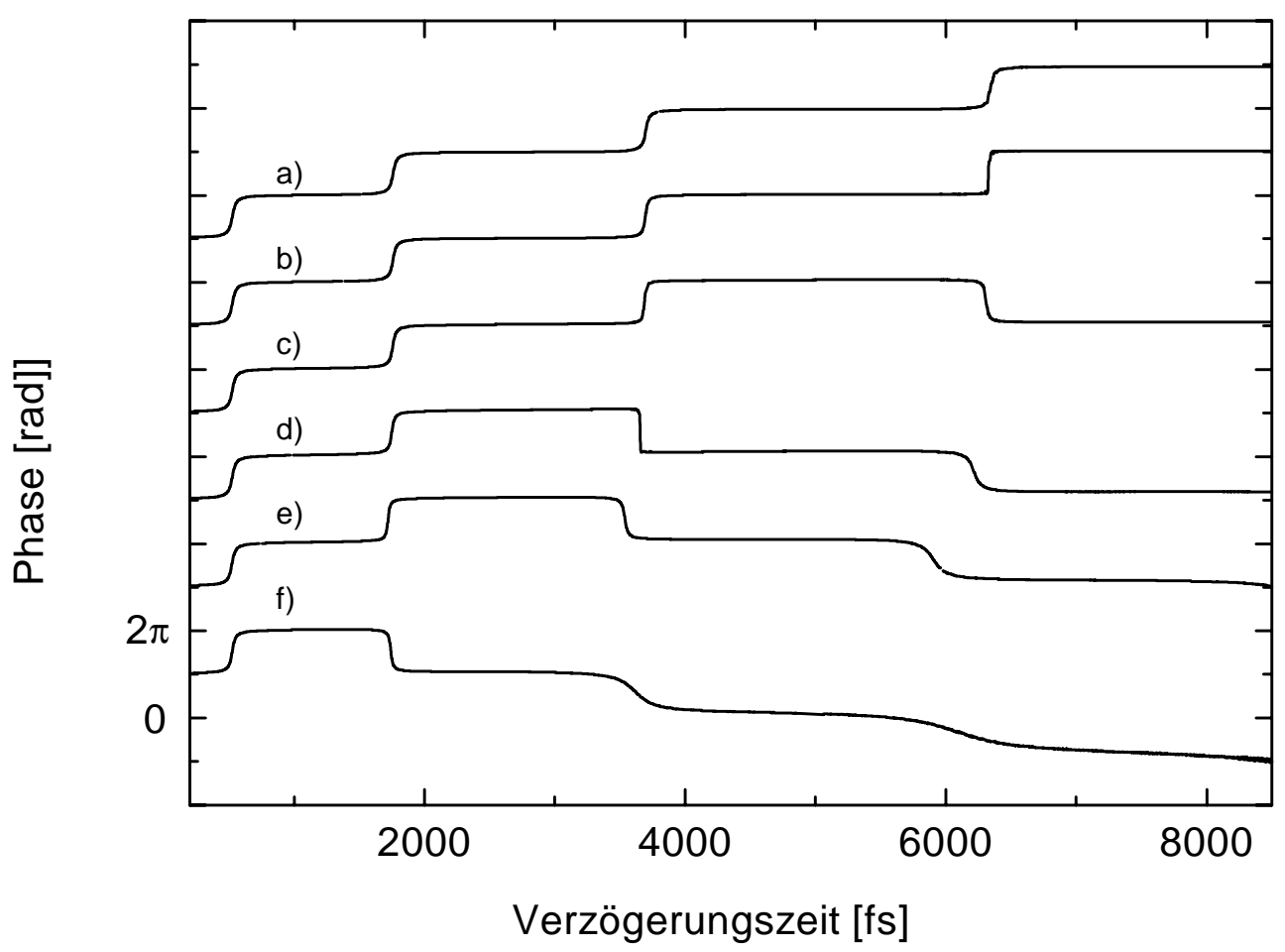

Abb. 4.13: Simulierte Phasenverläufe für eine asymmetrische Resonanz.

Parameter s. Tab. 4.1 . 
Für das Umklappen der Phase sind mehrere Kombinationen der Oszillatorparameter der Doppelresonanz möglich. Von den ursprünglich sechs Parametern sind die effektive Oszillatorstärke $\left(\mathrm{f}_{1}+\mathrm{f}_{2}=\mathrm{f}_{0}\right)$, die Resonanzfrequenz und die Dämpfung der Doppelresonanz durch das Experiment festgelegt, so daß drei Parameter frei wählbar sind. Mit dem Gewichtungsfaktor $\alpha$ der Oszillatorstärken $\mathrm{f}_{2}=\alpha \cdot \mathrm{f}_{0}\left(\right.$ d.h. $\left.\mathrm{f}_{1}=(1-\alpha) \cdot \mathrm{f}_{0}\right)$, dem Verhältnis der Dämpfungen $\beta=\gamma_{2} / \gamma_{1}$ und der relativen Verschiebung der beiden Resonanzfrequenzen $\Delta \omega=\omega_{2}-\omega_{1}$ erhält man die in Abb. 4.14 abgebildeten Parametertripel, die ein Phasenumklappen bei 4.3 ps bewirken. Der Bereich, in dem die drei Parameter frei wählbar sind, ist dadurch begrenzt, daß die Phase nicht umklappt (rechter Rand der Fläche) oder sich im Spektrum eine Doppelresonanz ausbildet (für größere Energieabstände als $\Delta \omega \approx 350 \mu \mathrm{eV}$ ).

Um ein quantitatives Maß für die Asymmetrie zu finden, wird das zweite, zentrale Moment der Differenzfunktion von linker zu rechter Flanke der Doppelresonanz gebildet:

$$
\mathrm{AS} \equiv \int_{0}^{\infty}\left(\varepsilon_{2}\left(\omega_{\mathrm{c}}-\omega\right)-\varepsilon_{2}\left(\omega_{\mathrm{c}}+\omega\right)\right) \cdot\left(\omega_{\mathrm{c}}-\omega\right)^{2} \mathrm{~d} \omega
$$

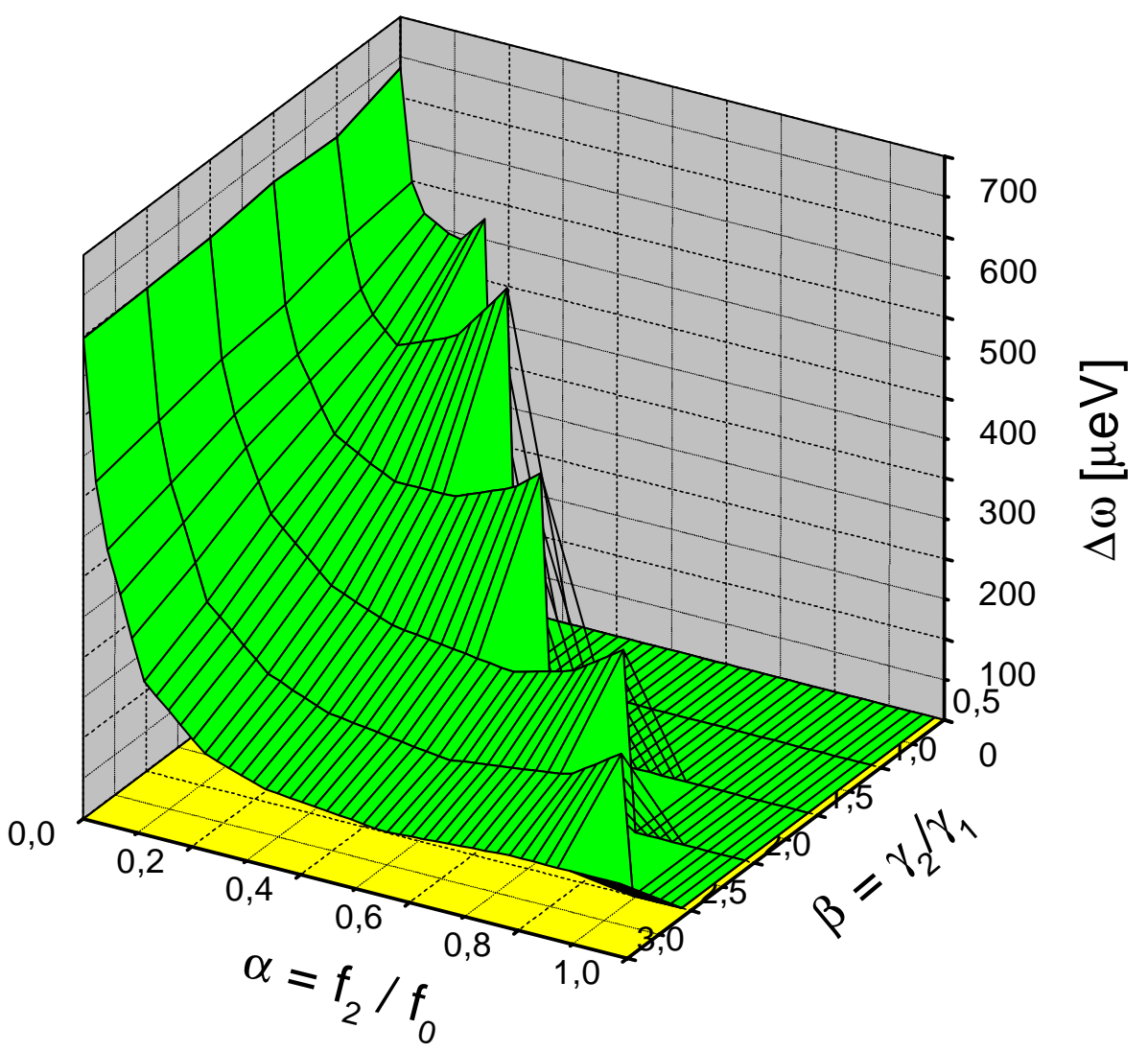

Abb. 4.14: Parametertripel der Doppelresonanz, für die der Phasensprung bei 4.3 ps stattfindet $\left(\hbar \omega_{\text {effektiv }}=1.515 \mathrm{eV}, \hbar \gamma_{\text {effektiv }}=60 \mu \mathrm{eV}, \mathrm{f}_{\text {effektiv }}=86 \mu \mathrm{eV}\right)$. 
wobei $\quad \varepsilon_{2}(\omega)=\varepsilon_{\mathrm{b}} \cdot \mathrm{f}_{0} / 2 \omega_{\mathrm{c}} \cdot\left(\frac{\gamma_{1} \cdot \alpha}{\left(\omega-\omega_{1}\right)^{2}+\gamma_{1}{ }^{2}}+\frac{\gamma_{2} \cdot(1-\alpha)}{\left(\omega-\omega_{2}\right)^{2}+\gamma_{2}{ }^{2}}\right)$

der lorentzförmige Imaginärteil der dielektrischen Funktion ist und die Zentralfrequenz gegeben ist durch $\omega_{c}=\left(\alpha \cdot \omega_{1}+(1-\alpha) \cdot \omega_{2}\right)$. Im Bereich $\alpha \in[0.1 \ldots 0.8]$ und $\gamma_{2} \in$ $\left[\gamma_{1} \ldots 3 \cdot \gamma_{1}\right]$ (wobei $\gamma_{1}$ derart gewählt ist, daß die effektive Dämpfung der Doppelresonanz $60 \mu \mathrm{eV}$ ist) beträgt die Asymmetrie, die das Umklappen bei 4.3 ps bewirkt, AS $=4 \cdot 10^{-11} \mathrm{eV}^{3}$.

Für die Beobachtung des Phaseneffekts ist entscheidend, daß die Phasensprünge, die gerade an Stellen auftreten, wo das elektrische Feld (fast) verschwindet, vollständig aufgelöst werden. Voraussetzung hierfür ist eine Meßmethode, die eine große Dynamik der Amplitude innerhalb einer Messung aufweist. 


\subsection{Anrege- und Testexperimente}

Für die Vertiefung des Verständnisses der Streuung des 1s-Exzitons am ungebundenen e-h-Plasma wurden Anrege- und Test-Experimente durchgeführt.

\subsubsection{Variation der zeitlichen Verzögerung zwischen Pump- und Testpuls}

Der zeitliche Abstand von Pump- und Testpuls wurde in einem Bereich von 0 bis 20 ps variiert, um den Einfluß der Relaxation der Ladungsträger zu untersuchen, die bei der genauen Bestimmung von Ladungsträgerdichten eingeht. Darüber hinaus können durch den zeitlichen Abstand kohärente Effekte ausgeschlossen werden.

In Abb. 4.15 ist der Amplitudenverlauf für zeitgleiches Auftreffen von Pump- und Testpuls sowie bei einem Abstand von 20 ps (Pumppuls vor Testpuls, beide spektral gleich) dargestellt. Die beiden Kurven liegen identisch übereinander.

Der Einfluß der zeitlichen Relaxation der Elektron-Loch-Kontinuumspaaren ist für die Amplitude und Phase der Polariton-Pulspropagation auf der ps-Skala zu vernachlässigen. Sowohl die Dämpfung als auch das charakteristische Verhalten der Phase wird allein durch die Anwesenheit der Ladungsträger hervorgerufen. 
Zur Überprüfung wird der Pumppuls 20 ps hinter den Testpuls plaziert. Die Streurate nimmt innerhalb der Meßgenauigkeit auf den Wert ohne Pumppuls $a b^{23}$.

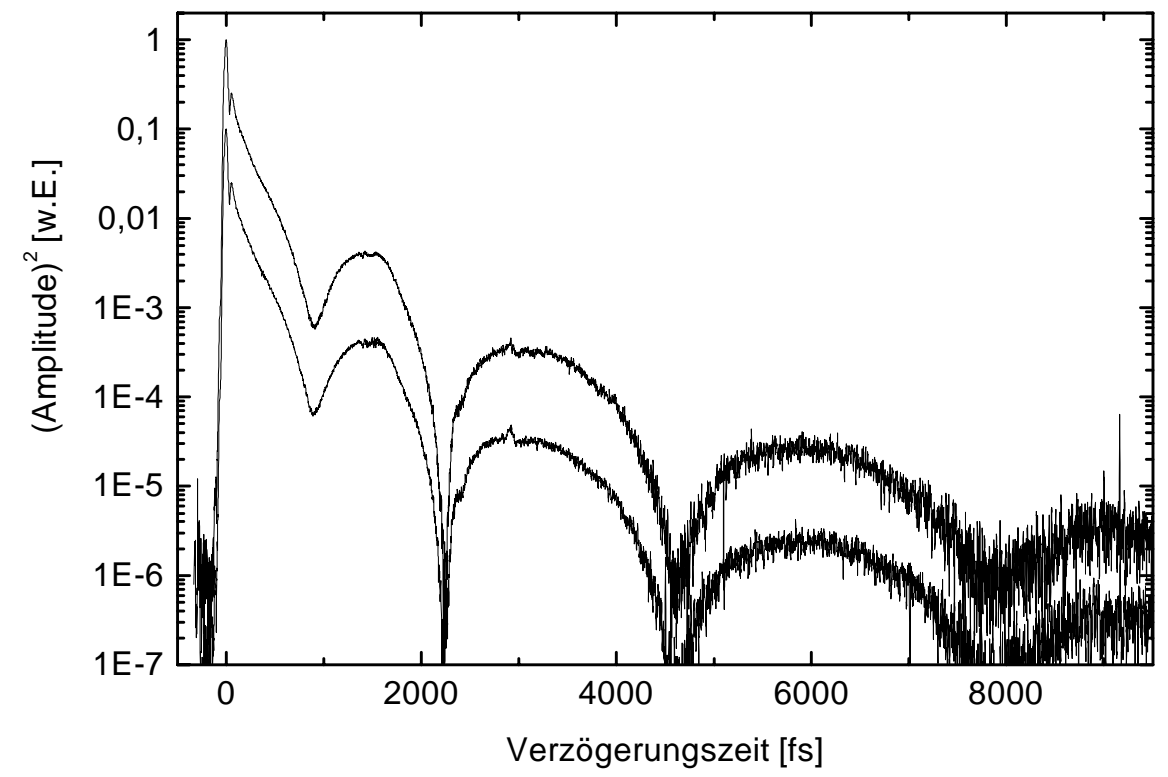

Abb. 4.15 : Gemessene Amplitudenverläufe mit dem Pumppuls zeitgleich bzw. 20 ps vor dem Testpuls. Die kleine Modulation bei 2.9 ps ist ein Reflex der Glastasche von der Prohe

Für tiefe Temperaturen wird die mittlere Energieverlustrate der Elektronen und Löcher als Funktion der Ladungsträgertemperaturen bei $\mathrm{T}_{\text {Gitter }}=1,8 \mathrm{~K}$ bestimmt [84]. Während für tiefe Temperaturen der Exzitonen akustische Deformationspotentialstreuung (besonders für Löcher) überwiegt, werden die Raten für höhere Temperaturen von LO-Phonon-Streuung dominiert. Wie man in Abb. 4.16 sieht, ist der Energieverlust der Ladungsträger nach Abschluß der Relaxation durch LO-Phononen auf der ps-Zeitskala vernachlässigbar. Für Anregungen mit einem fs-Puls nahe der Bandlückenenergie sind Temperaturen von 30 bis $70 \mathrm{~K}$ für die Ladungsträger realistisch.

\footnotetext{
${ }^{23}$ Bei einer Repetitionsrate von $80 \mathrm{MHz}$ des Lasers und einer mittleren Lebensdauer von unter $1 \mathrm{~ns}$ ist nur ein Anteil von rund maximal $10^{-5}$ der vom vorherigen Puls generierten Ladungsträger übrig, welcher nicht mehr nachgewiesen werden kann.
} 


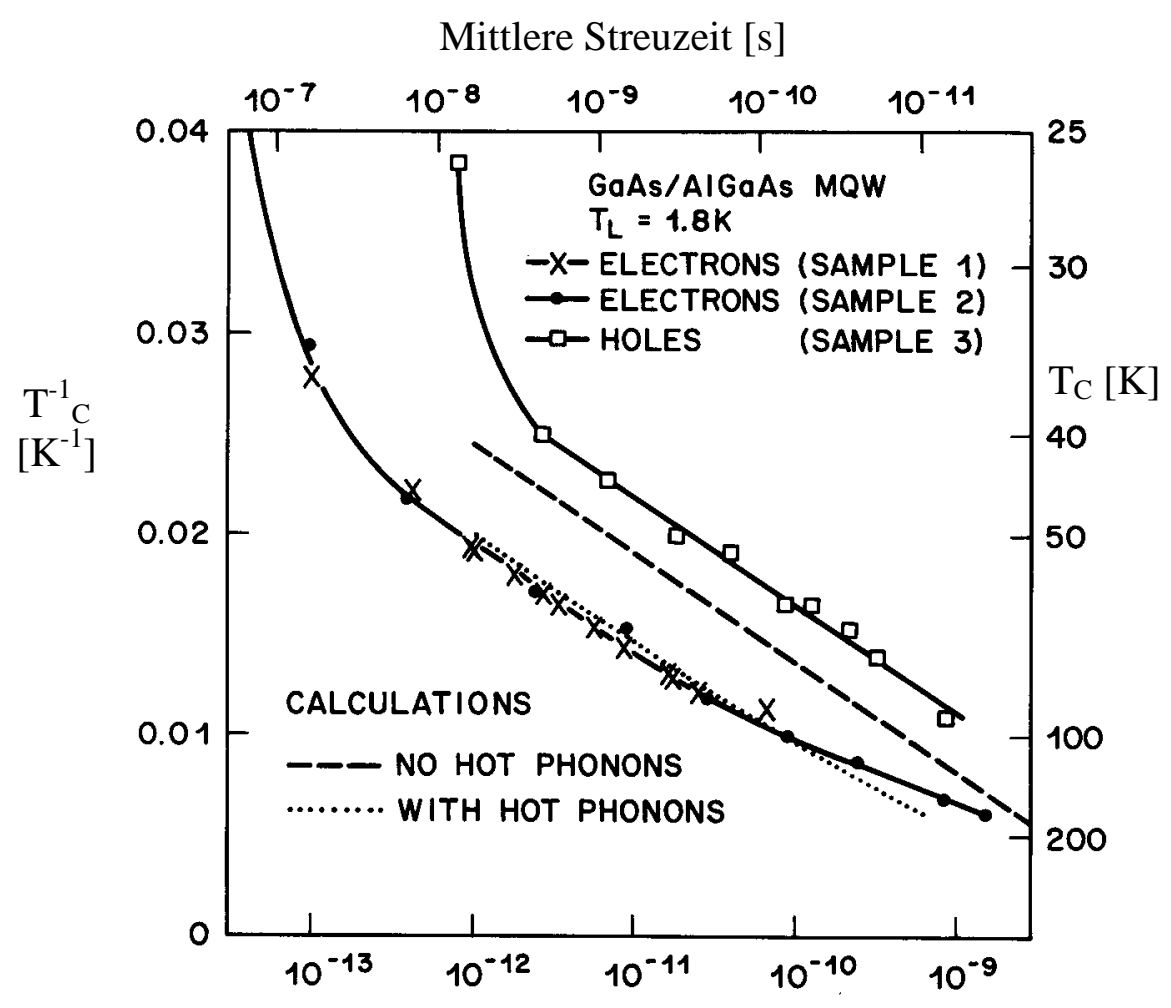

Energieverlustrate pro Teilchen [W]

Abb. 4.16: Gemessene Energieverlustraten an das Gitter für Elektronen und Löcher als Funktion der Ladungsträgertemperaturen bei einer Gittertemperatur $\mathrm{T}_{\text {Gitter }}=1,8 \mathrm{~K}$ [84].

\subsubsection{Variation des Pumpspektrums}

Um den Einfluß der energetischen Lage der optisch angeregten Elektron-LochKontinuumspaare auf die Polariton-Pulspropagation $\mathrm{zu}$ untersuchen, wurde das Spektrum der Pumppulse variiert. Sie wurden mit Hilfe eines Pulsformers erzeugt (s. Kap. 3.3), hatten eine spektrale Breite von rund $3 \mathrm{meV}$ und wurden ca. 10 ps vor dem Testpuls plaziert. Für eine fest eingestellte Intensität des Pumpstrahles wurde die 1s-Exzitondämpfung in Abhängigkeit von der Zentralfrequenz des Pumppulses bestimmt. Der Strahl des Pumppulses wurde leicht defokussiert, um sicherzustellen, daß sich das Testvolumen innerhalb des Anregevolumens befindet. Die Resultate für zwei Anregungsfluenzen (300 und $150 \mu \mathrm{W}$ ) sind in Abb. 4.17 aufgetragen.

Die Dämpfung nähert sich einem festen Wert für steigende Pumpfrequenzen, der für verschiedene Anregungsdichten unterschiedlich ist.

Diese Beobachtung läßt sich durch die Streuung der Elektron-Lochpaare an LO-Phononen erklären, die für eine LO-Phononenenergie $\left(\mathrm{E}_{\mathrm{LO}}=36 \mathrm{meV}\right)$ oberhalb der 
Bandlückenenergie sehr effizient und schnell ist [85]. Für Ladungsträgerpaarenergien unterhalb dieser Schwelle $\left(\mathrm{E}<\mathrm{E}_{\mathrm{LO}}+\mathrm{E}_{\mathrm{gap}}\right.$ ) liegen Dämpfungszeiten wegen der schwachen Kopplung an akustische Phononen bei 100 ps bis $1 \mathrm{~ns}$, oberhalb um drei Größenordnungen kleiner. Entsprechend relaxieren energetisch oberhalb der Schwelle angeregte Ladungsträger schnell zur Bandkante, an der sie dann zur Ruhe kommen (s. Energieverlustraten s. Abb. 4.16).

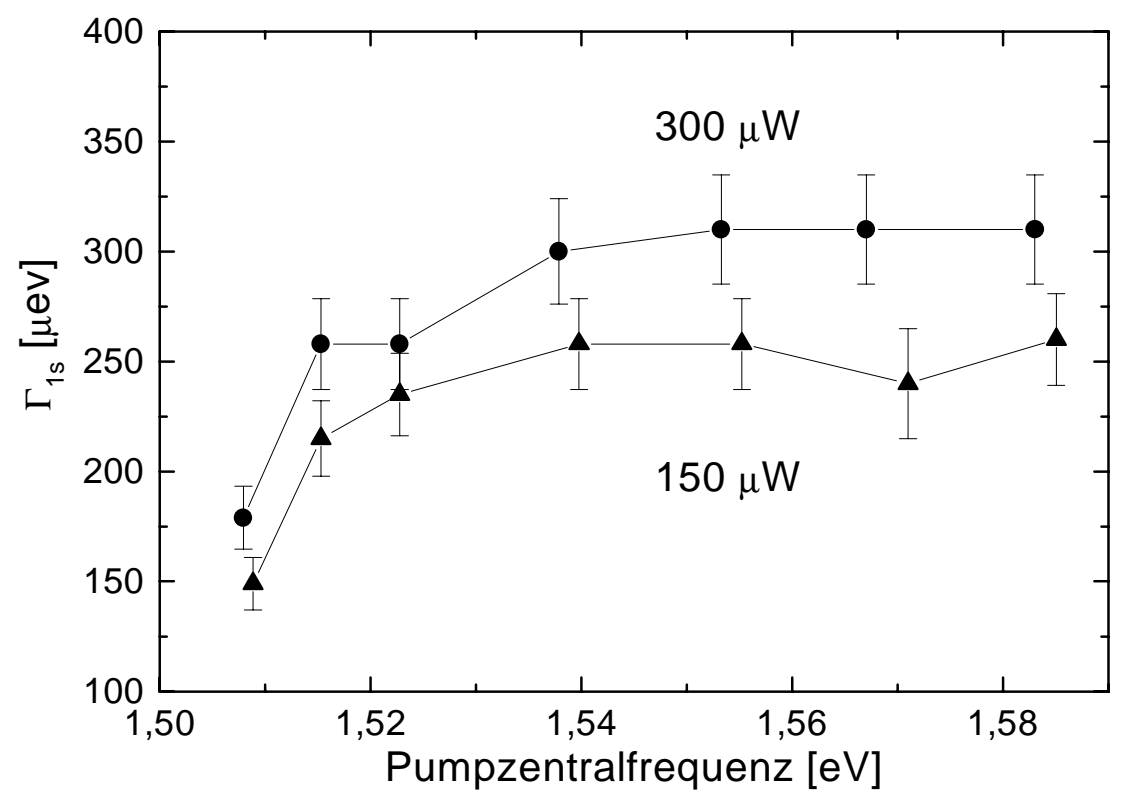

Abb. 4.17 : Im Rahmen des Modells 1 aus den gemessenen Amplitudenkurven bestimmte Linienbreiten in Abhängigkeit von der Zentralfrequenz des Pumppulses für zwei verschiedene Laserintensitäten.

Die Dämpfungskonstante, die bei der Polariton-Pulspropagation beobachtet wird, ist somit gegeben durch die relaxierte Verteilung der freien Elektronen und Löcher, die sich nach Abschluß der Streuung an LO-Phononen einstellt.

Die Dämpfung des 1s-Exzitonen konvergiert mit steigender Zentralfrequenz des Pumppulses gegen einen festen Wert, der einerseits von der Anregungsdichte, anderseits von der Temperatur des von den Elektron-Lochpaaren eingenommenen Zwischenzustandes bestimmt ist.

Die Beobachtung motiviert die zuvor im Kap. 4.1.3.1 unternommene Einteilung in Exzitondichte bzw. Kontinuumsladungsträgerdichte, die keine weitere Rücksicht auf die energetische Verteilung der Ladungsträger in den jeweiligen Bändern macht. Die Konvergenz gegen einen festen Wert rechtfertigt es indirekt, den Einfluß der nichtthermischen Phononen auf die Abhängigkeit der Dämpfung von der Anregungsdichte zu vernachlässigen. 
$\mathrm{Zu}$ bemerken ist, daß die schnelle Relaxation über LO-Phononen nicht im Signal für kleine Zeiten abgelesen werden kann: Das Meßsignal ist eine integrale Größe, die wegen der langen, dem System inhärenten Antwortzeit und der Verknüpfung von verschiedenen Raumzeitpunkten durch die Propagation über schnelle Prozesse mittelt. Ein schneller ${ }^{24}$ Prozeß in der Probe verändert das zeitliche Signal für alle Zeiten und nicht nur für kurze Zeiten.

${ }^{24}$ Schnell im Vergleich zur Relaxationszeit des Exzitons 


\section{Zusammenfassung}

Die vorliegende Arbeit beschäftigt sich im wesentlichen mit zwei Aufgabenstellungen:

Zum einen wurde die Methode der interferometrischen Kreuzkorrelation zur Bestimmung der Amplitude und Phase einer optischen Übertragungsfunktion mit Zeitauflösung im fs-Bereich für den sichtbaren Spektralbereich weiterentwickelt. Wie man an der Vielzahl der Publikationen in den letzten Jahren erkennt, ist das Interesse an amplituden- und phasenmessenden Methoden groß. Die vorgestellte Methode beruht auf einem linearen Detektionsmechanismus, der höchste Sensitivität hinsichtlich der minimal notwendigen mittleren Leistung ermöglicht. Die derzeitig realisierte Apparatur erreicht eine Dynamik innerhalb eines Meßlaufes von 3.5 Größenordnungen im Korrelationssignal, welches proportional zur Amplitude des elektrischen Feld des Signalpulses ist. Hiermit ist es möglich, die Phase an Zeitpunkten aufzulösen (z.B. in Schwebungsminima), an denen die Amplitude des elektrischen Feldes fast verschwindet.

Zum anderen wurde mit Hilfe der Methode der interferometrischen Kreuzkorrelation die Polariton-Pulspropagation in der 1s-Exzitonresonanz in Volumen Galliumarsenid bei niedrigen und mittleren Anregungsdichten an Elektron-Loch-Kontinuumspaaren bei tiefen Temperaturen untersucht. Hierfür stand eine hochreine Volumen Probe zur Verfügung, die minimale homogene Linienbreiten aufwies, und so Präzisionsmessungen in Abhängigkeit der Kontrollparameter ermöglichte.

Bei niedrigen Anregungsdichten wird die Schwebung zwischen dem 1s- und 2sExzitonzustand beobachtet und eine gute Übereinstimmung mit der Theorie im Rahmen der Beschreibung des Halbleiters durch eine Lorentz verbreiterte dielektrische Funktion gefunden. Die Oszillatorstärke der 1s-Resonanz wird zu $86 \pm$ $5 \mu \mathrm{eV}$ bestimmt. Gegenstand des Interesses war die Streuung des 1s-Exzitons an Elektron-Loch-Kontinuumpaaren. Unter Annahme eines Modells, welches das Ladungsträgerprofil berücksichtigt, das durch den Absorptionskoeffizienten aufgeprägt wird, steigt die Dämpfung des 1s-Exzitons sublinear mit der Fluenz an, welches im Widerspruch zu einfachen Streumodellen steht.

Die Polariton-Pulspropagation wurde erstmals phasenaufgelöst gemessen und eine charakteristische Abhängigkeit der Phase von der Anregungsdichte beobachtet, die schon bei geringen Anregungsdichten von $6 \cdot 10^{13} \mathrm{~cm}^{-3}$ einsetzt. Dies ist in zweifacher 
Weise interessant: Einerseits wurde bisher wenig über Phaseneffekte trotz der in letzter Zeit entwickelten, zahlreichen Methoden zur Phasenmessung auf der fsZeitskala berichtet. Anderseits konnten damit Veränderungen in der Linienform des 1s-Exzitons bei sehr viel kleineren Anregungsdichten festgestellt werden (rund 1.5 Größenordnungen), als in der Literatur berichtet wurde. Der Phaseneffekt wird auf eine durch Ladungsträger induzierte Asymmetrie der Exzitonresonanz zurückgeführt, die zu einer verstärkten Absorption auf der Hochenergieflanke führt. 


\section{Wichtige Abkürzungen und Konstanten:}

$\begin{array}{ll}\alpha(\omega) & \text { Absorptionskoeffizient } \\ \mathrm{a}_{\mathrm{B}} & \text { Bohrradius } \\ \chi(\omega) & \text { dielektrische Suszeptibilität } \\ \mathrm{d} & \text { Probendicke } \\ \mathrm{E} & \text { Energie, elektrisches Feld } \\ \mathrm{E}_{\mathrm{g}} & \text { Bandlückenenergie } \\ \varepsilon_{0} & \text { Dielektrizitätskonstante des Vakuums } \\ \varepsilon_{\mathrm{B}} & \text { Dielektrizitätskonstante des Hintergrunds } \\ \mathrm{f} & \text { Oszillatorstärke } \\ \mathrm{F} & \text { Fluenz } \\ \gamma, \Gamma & \text { Dämpfungskonstante, halbe Halbwertsbreite einer Lorentzlinie } \\ \widetilde{\mathrm{n}} & \text { komplexer Brechungsindex } \\ \mathrm{n}_{\mathrm{k} \text { ont }} & \text { Anregungsdichte im Kontinuum } \\ \omega & \text { Kreisfrequenz } \\ \omega_{0} & \text { Kreisfrequenz der Resonanz } \\ \omega_{\mathrm{T}} & \text { transversale Frequenz } \\ \omega_{\mathrm{L}} & \text { longitudinale Frequenz } \\ \omega_{\mathrm{LT}} & \text { Frequenz der longitudinal/transversal Aufspaltung } \\ \mathrm{Ry} & \text { Exzitonbindungsenergie (Rydbergenergie) } \\ \psi(\mathrm{x}) & \text { Wellenfunktion, Digammafunktion }\end{array}$

\section{Konstanten von Galliumarsenid:}

$\begin{array}{lll}\mathrm{a}_{\mathrm{B}} & 13 \mathrm{~nm} & {[85]} \\ \alpha(\mathrm{E}=1.52 \mathrm{eV}) & 0.8 \mu \mathrm{m}^{-1} & {[34]} \\ \varepsilon_{\mathrm{b}}{ }^{*} & 12.55 & {[34]} \\ \mathrm{m}_{\mathrm{e}}{ }^{*} & 0.067 \cdot \mathrm{m}_{0} & {[86]} \\ \mathrm{m}_{\mathrm{hl}}{ }^{*} & (0.082 \pm 0.004) \cdot \mathrm{m}_{0} & {[86]} \\ \mathrm{m}_{\mathrm{hh}}{ }^{*} & (0.067 \pm 0.02) \cdot \mathrm{m}_{0} & {[86]} \\ \mathrm{m}_{\mathrm{h}} & 0.325 \cdot \mathrm{m}_{0}, & \\ & , \text { optisch gemittelt über } \mathrm{m}_{\mathrm{lh}} \text { und } \mathrm{m}_{\mathrm{hh}}{ }^{*} & {[34]} \\ \hbar \omega_{\mathrm{LT}} & (86 \pm 5) \mu \mathrm{eV} & \text { in dieser Arbeit } \\ \mathrm{Ry} & (4,2 \pm 0.02) \mathrm{meV} & {[34]} \\ \mathrm{E}_{\mathrm{g}} & (1.5192 \pm 0.02) \mathrm{meV} & {[87]}\end{array}$

\section{Andere verwendete Konstanten [40]:}

$\begin{array}{lll}\mathrm{c} & \text { Lichtgeschwindigkeit } & 2,9979 \cdot 10^{8} \mathrm{~m} / \mathrm{s} \\ \mathrm{e} & \text { Elementarladung } & 1,6022 \cdot 10^{-19} \mathrm{C} \\ \varepsilon_{0} & \text { Dielektrizitätskonstante des Vakuums } & 8,8542 \cdot 10^{-12} \mathrm{As} / \mathrm{Vm} \\ \hbar & \text { Plancksches Wirkungsquantum } & 6,6262 \cdot 10^{-34} \mathrm{Js} / 2 \pi \\ \mathrm{m}_{0} & \text { Ruhemasse des Elektrons } & 9,1095 \cdot 10^{-31} \mathrm{~kg}\end{array}$




\section{Literaturverzeichnis:}

[1] R.G.Ulbrich, G.W.Fehrenbach, Phys.Rev.Lett. 43, 963 (1981)

[2] Kazuhiro Ema and Makoto Kuwata-Gonokami, Phys.Rev.Lett. 75, 224 (1995)

[3] D.Fröhlich, A.Kulik, B.Uebbing, A. Mysyrowicz, V.Langer, H.Stolz, W.von der Osten, Phys.Rev.Lett. 67, 2343 (1991)

[4] S.Nüsse, P.Haring Bolivar, and H.Kurz, F.Levy, A.Chevy, O.Lang, Phys.Rev. B 55, 4620 (1997)

[5] U.Neukirch and K.Wundke, Phys.Rev. B 55, 15408 (1997)

[6] J.D.Jackson, Klassische Elektrodynamik, 2. Auflage, New York: de Gruyter (1983)

[7] H.Haug, and S.W.Koch, Quantum Theory of the Optical and Electronic Properties of Semiconductors, 2nd Ed., Singapore: World Scientific (1993)

[8] V.M.Agranovich und V.L.Ginzburg, Crystal Optics with Spatial Dispersion and Excitons, 2. Aufl. Springer Series in Solid-State Sciences 42 (1884)

[9] C.F.Klingshirn, Semiconductor Optics, Berlin: Springer (1995)

[10] C.Cohen-Tannoudji, B.Diu, F.Laloë, Quantum Mechanics, Vol. I und II, Wiley (1977)

[11] E.O.Kane, J.Phys.Chem.Solids 1, 249 (1957)

[12] R.J.Elliot, Phys.Rev. 108, 1384 (1957)

[13] I.S.Gradshteyn \& I.M. Ryshik, Tables of Integrals, Series, and Products, 5th edn., Academic press, San Diego (1994)

[14] C.Tanguy, Phys.Rev.Lett. 75, 4090 (1995)

[15] L.Bányai, Q.T.Vu, B.Bieck, and H.Haug,, Phys.Rev.Lett 81, 882 (1998)

[16] C.Tanguy, IEEE J.Quant.Elec., 32, 1746 (1996)

[17] J.J.Hopfield, Phys.Rev.Lett. 112, 1555 (1958)

[18] J.J.Hopfield, Phys.Rev.Lett. 132,563 (1963)

[19] A.Stahl und I.Balslev, Electrodynamics of the Semiconductor Band Edge, Springer Tracts Modern Physics Vol. 110 (1987)

[20] M.Born, K.Huang (1954)

[21] N.W.Ashcroft, N.D.Mermin, Solid State Physics, International Edition, Saunders 1976

[22] A.Tredicucci, Y.Chen, and F.Basani, Phys.Rev. B 47, 10348 (1993)

[23] R.G.Ulbrich and C.Weisbuch, Phys.Rev.Lett. 38, 865 (1977)

[24] S.I.Pekar, Zh.Eksp.Teor.Fiz. 33, 1022 (1957) [Soviet Phys. JETP 6, 785 (1958)]

[25] P.Halevi, Spatial Dispersion in Solids and Plasma (North-Holland, Amsterdam, 1992); P.Halevi in Excitons in Confined Systems, R.DelSole (Ed.), Springer Proceedings in Physikcs Vol. 25 (Springer-Verlag Berlin, 1988); P.Halevi,G.Hernandez-Cocoletzi, Phys.Rev.Lett. 48,1500 (1982)

[26] F.Forstmann and H.Stenschke, Phys.Rev.B 17, 1489 (1978); F.Forstmann,Z.Phys. 32, 3851 (1979)

[27] C.S.Ting, M.J.Frankel, and J.L.Birman, Solid State Commun. 17, 1285 (1975)

[28] K.Victor, V.M.Axt, A.Stahl, Zeitschrift für Physik B 92, 35 (1993)

[29] H.Bakker, S.Hunsche, and H.Kurz, Phys.Rev.Lett. 69, 2823 (1992)

[30] Y.Segawa, Y.Aoyagi and S.Mamba, Solid State Commun. 32, 229 (1979) 
[31] D.Weber, W.Petri, C.F.Klingshirn, S.Shevel, E.O.Göbel, Phys.Rev. B 55, 12848 (1997)

[32] A.Knorr, R.Binder, M.Lindberg, and S.W.Koch, 46 , 7179 (1992)

[33] D.S.Kim et al., Phys.Rev.B 48, 17902 (1993)

[34] M.Trinn, Dissertation, Göttingen (1992)

[35] A.Armitage, T.A.Fisher, M.S.Skolnick, D.M.Whittaker, and P.Kinser, Phys.Rev.B 55, 55 (1997)

[36] H.Giessen, A.Knorr, S.Haas, S.W.Koch, S.Linden, J.Kuhl, M.Hetterich, M.Grun, C.Klingshirn, Phys. Rev. Lett. 81, 4260 (1998)

[37] T.Katsuyama and K.Ogawa, J.Appl.Phys. 75, 7607 (1994)

[38] G. Panzarini, L.C. Andreani, Solid State Comm. 102, 505 (1997)

[39] L.Brillouin, Wave Propagation and Group Velocity, Academic Press (1960)

[40] H.Ibach und H.Lüth, Festkörperphysik, Springer-Verlga Berlin (1980)

[41] E.Göbel, Festkörperprobleme 30 (1990)

[42] A.Mysyrowic, D.Hulin, Antonetti, A.Migus, W.T.Masselink, and H.M.Morkoc, Phys.Rev.Lett. 56, 2748 (1986) ; A.v.Lehmen, D.S.Chemla, J.E.Zucker, J.P.Heritage, Opt.Lett. 11, 609 (1986)

[43] V.Langer, H.Stolz, and W.von der Osten, Phys.Rev.Lett. 64, 854 (1990)

[44] s.z.B.: H.J.Eichler, P.Günther, and D.W.Pohl, Laser Induced Dynamical Gratings, Springer Series Optical Sciences, Vol 50 (1986)

[45] L.Bányai, D.B.TranThoai, E.Reitsamer, H.Haug, D.Steinbach, M.U.Wehner, M.Wegener, T.Marschner, and W.Stolz, Phys.Rev.Lett 75, 2188 (1995)

[46] J-Shah. Sol. State Com. 32. 1051 (1989); J.Shah, Superlatt.\&Microstr. 6, 293 (1989)

[47] K.Leo,M.Wegener, J.Shah, D.S.Chemla, E.O.Göbel, and T.C.Damen, S.Schmitt-Rink, W.Schäfer, Phys.Rev.Lett. 65, 1340 (1990)

[48] Y.Z.Hu, R.Binder, and S.W.Koch, Phys.Rev.B 49, 14382 (1994)

[49] F.Rossi, S.Haas, and T.Kuhn, Phys.Rev.Lett. 72, 152 (1994)

[50] S.Hughes, A.Knorr, S.W.Koch, R.Binder, R.Indik, and J.V.Moloney, Solid State Commun. 100, 555 (1996)

[51] F.Jahnke, M.Kira, S.W.Koch, G.Khitrova, E.K.Lindmark, T.R.Nelson, Jr., D.V.Wick, J.D.Berger, O.Lyngnes, H.M.Gibbs, and K.Tai, Phys. Rev. Lett. 77, 5257 (1996)

[52] A.Girndt, F.Jahnke, A.Knorr, S.W.Koch, and W.W.Chow, Phys.Stat. Sol. B 202, 725 (1997)

[53] C.Trallero-Giner, R.Zimmermann, M.Trinn, R.G.Ulbrich, Proc. 23rd Int. Conf. On the Physics of Semiconductors Vol.I, Berlin (1996)

[54] K.Naganuma, K.Mogi, and H.Yamada, IEEE J.of Quant.Electr. 25, 1225 (1989)

[55] R.L.Fork, O.E.Martinez, J.P.Gordon, Opt. Lett. 9, 150 (1984)

[56] A.M.Weiner, J.P.Heritage, and E.M. Kirschner, J.Opt.Soc.Am. B 5,1563 (1988)

[57] R.J.Bell, Introductory Fourier Transform Spectroscopy, Academic Press, New York (1972)

[58] K.W.DeLong, R.Trebino, J.Hunter and W.E.White, J.Opt. Soc.Am. B 11, 2206 (1994)

[59] D.S.Kim, J.Shah, D.A.B.Miller, and T.C.Damen, W.Schäfer, L.Pfeiffer, Phys.Rev. B 48, 17902 (1993)

[60] D.N.Fittinghoff, J.L.Bowie, J.N.Sweetser, R.T.Jennings, M.A.Krumbügel, K.W.Delong, R.Trebino, and I.A.Walmsley, Opt.Lett. 21, 884 (1996) 
[61] D.S.Chemla et al. Phys.Rev.B 50, 8439 (1994)

[62] Entwicklung und Programmierung von G.Böhne.

[63] W.Lauterborn, T.Kurz, M.Wiesenfeld, Coherent Optics- Fundamentals and Applications, Springer (1993)

[64] G.Böhne, S,.Freundt, S.Arlt, D.Pfister, C.Nacke, J.S.Nägerl, R.G.Ulbrich, phys.stat.sol (b) 188, $321(1995)$

[65] G.Böhne, S.Freundt, S.Arlt, D.Pfister, C.Nacke, J.S.Nägerl and R.G.Ulbrich, Proc. 22nd Int.Conf.Physics of Semiconductors, (World Scientific, Singapore 1995), p-357

[66] D.Fröhlich, A.Kulik, and B.Uebbing, A.Mysyrowicz, V.Langer, H.Stolz, and W.von der Osten Phy.Rev.Lett. 67, 2343 (1992)

[67] J.S.Nägerl, T.Reker, G.Böhne, R.G.Ulbrich, Phys.Stat.Sol (b) 206, 357 (1998), T.Reker, Diplomarbeit Göttingen (1997)

[68] A.C.Schaefer, D.G.Steel, Phys.Rev.Lett 79, 4870 (1997)

[69] G.Fishman, Sol.St.Com. 27, 1097 (1978); R.G.Ulbrich, C.Weisbuch, Phys.Rev.Lett. 38, 865 (1977)

[70] R.G.Ulbrich, in: Materials Science \& Technology, Vol. 4 (Electronic Structure and Properties of Semiconductors) VCH Verlagsgesellschaft, Weinheim (1991)

[71] E.O.Kane, Phys.Rev. B 11, 3850 (1975)

[72] M.Schreiber and Y.Toyozawa, J.of the Phys.Soc.of Jap. 51, 1528 (1982)

[73] C.W.Greeff, H.R.Glyde, Phys.Rev.B 51, 1778 (1995)

[74] B.Stabenau, Diplomarbeit, Göttingen (1998)

[75] L.Schultheis, J.Kuhl, and A.Honold, and C.W.Tu, Phys.Rev.Lett. 57, 1635 (1986)

[76] T.Witte, Diplomarbeit Göttingen (1999)

[77] G.R.Allan and H.M. van Driel, Phys.Rev. B 59, 15740 (1999)

[78] U.Neukirch and K.Wundke, Phys.Rev.B 55, 15408 (1997)

[79] L.Schultheis, J.Kuhl, and A.Honold, Phys.Rev.Lett. 57, 1635 (1986)

[80] A.Honold, L.Schultheis, J.Kuhl, Phys.Rev.B 40, 6442 (1989)

[81] H.Wang, K.B.Ferrio, and D.G.Steel, P.R.Berman, Y.Z.Hu, R.Binder, and S.W.Koch, Phys.Rev. A 49, R1551 (1994); H.Wang, K.B.Ferrio, and D.G.Steel, Y.Z.Hu, R.Binder, and S.W.Koch, Phys.Rev.Lett. 71, 1261 (1993)

[82] G.W.Fehrenbach, W.Schäfer, and R.G.Ulbrich, J.of Luminescence 30, 154 (1985)

[83] J.S.Nägerl, B.Stabenau, G.Böhne, R.G.Ulbrich, G.Manzke, K.Henneberger, erscheint in phys. stat. sol. (b), (2000)

[84] J.Shah, Hot Carriers in Semiconductors Nanostructures, Academic Press (1992)

[85] R.G.Ulbrich, „Dynamics of Optical Excitations in Semiconductors“ , in Confined Elctrons and Photons, E.Burstein and C.Weisbruch (eds), PlenumPress, New York (1995)

[86] J.S.Blakemore, J.Appl.Phys. 53, 10 (1982)

[87] C.Weisbuch, R.G.Ulbrich, in Light Scattering in Soldis III, ed. M.Cardona, G.Güntherodt, S.218.Springer (1982) 


\section{Danksagung}

An dieser Stelle möchte ich all jenen meinen Dank aussprechen, die durch ihre Hilfe und Unterstützung zum Gelingen dieser Arbeit beigetragen haben.

Herrn Prof. Dr. R.G. Ulbrich danke ich für die vielen konstruktiven Fragen, für die Diskussionen und seine Anregungen.

Herrn Prof. Dr. D. Ronneberger danke ich für die Übernahme des Korreferats.

Mein besonderer Dank richtet sich an Gregor Böhne, der in zahlreichen Diskussionen unermüdlich mit Rat und Tat zur Seite stand.

Meinen Freunden aus dem Femtolabor Ralf Ziebold, Thomas Witte, Birgit Stabenau und Tobias Reker danke ich herzlich. Mit Euch hat die gemeinsame Zeit im Labor großen Spaß gemacht! Auch möchte ich mich bei allen anderen aus der Arbeitsgruppe - den Tunnlern, den Snommern, den Ramanikern und den PLern - für die gute Zusammenarbeit und Hilfsbereitschaft bedanken.

Martin Wenderoth, Martin Hübner, Thomas Östreich und Günter Manzke sei großer Dank für die fruchtbaren Diskussionen.

Ein großer Dank gebührt Herrn Schulte und Edith Rohrmoser für die Hilfe bei organisatorischen Fragen sowie Herrn Sonnenberg und Herrn Langohr stellvertretend für die Mechanik- und die Elektronikwerkstatt.

Meiner lieben Frau Monica danke ich für die viele Geduld, Unterstützung und Liebe, die sie mir stets entgegenbringt.

Allen sei nochmals herzlich Dank! 